\title{
Republic of Mozambique: 2009 Article IV Consultation, Fourth Review Under the Policy Support Instrument, and Request for a Twelve-Month Arrangement Under the Exogenous Shocks Facility - Staff Report; Public Information Notice and Press Release on the Executive Board Discussion; and Statement by the Executive Director for the Republic of Mozambique
}

Under Article IV of the IMF's Articles of Agreement, the IMF holds bilateral discussions with members, usually every year. In the context of a combined discussion of the 2009 Article IV consultation with the Republic of Mozambique and the fourth review under the Policy Support Instrument, and request for a twelve-month arrangement under the exogenous Shocks Facility, the following documents have been released and are included in this package:

- $\quad$ The staff report for the combined 2009 Article IV Consultation, Fourth Review Under the Policy Support Instrument, and Request for a Twelve-Month Arrangement Under the Exogenous Shocks Facility, prepared by a staff team of the IMF, following discussions that ended on May 13, 2009, with the officials of the Republic of Mozambique on economic developments and policies. Based on information available at the time of these discussions, the staff report was completed on June 19, 2009. The views expressed in the staff report are those of the staff team and do not necessarily reflect the views of the Executive Board of the IMF.

- $\quad$ A Public Information Notice (PIN) and a Press Release, summarizing the views of the Executive Board as expressed during its June 30, 2009, discussion of the staff report on issues related to the Article IV consultation and the IMF arrangement, respectively.

- $\quad$ A statement by the Executive Director for the Republic of Mozambique.

The documents listed below have been or will be separately released.

Letter of Intent sent to the IMF by the authorities of the Republic of Mozambique*

Memorandum of Economic and Financial Policies by the authorities of the Republic of Mozambique*

Technical Memorandum of Understanding*

*Also included in Staff Report

The policy of publication of staff reports and other documents allows for the deletion of market-sensitive information.

Copies of this report are available to the public from

International Monetary Fund • Publication Services

$70019^{\text {th }}$ Street, N.W. • Washington, D.C. 20431

Telephone: (202) 623-7430 • Telefax: (202) 623-7201

E-mail: publications@imf.org • Internet: http://www.imf.org

International Monetary Fund

Washington, D.C. 

INTERNATIONAL MONETARY FUND

REPUBLIC OF MOZAMBIQUE

\section{Staff Report for the 2009 Article IV Consultation, Fourth Review Under the Policy Support Instrument, and Request for a Twelve Month Arrangement Under the Exogenous Shocks Facility}

Prepared by the African Department

(In consultation with other departments)

Approved by Mark Plant and Dominique Desruelle

June 19, 2009

- Discussions for the 2009 Article IV consultations and the fourth review of the Policy Support Instrument (PSI) were held in Maputo April 28-May 13. The mission team consisted of Mr. Sharer (head), Mr. Staines, Mr. Rosa, Ms. Ocampos (all AFR), Mr. Vitek (SPR), Mr. Fischer (resident representative), and Ms. Bosten (resident representative office). Mr. Demekas (MCM, FSAP mission chief) also joined the mission to discuss the findings of the FSAP Update. Mr. Sulemane (OEDAE) participated in the policy discussions, which Mr. Nucifora (World Bank) joined.

- The mission met with the Prime Minister; the Ministers of Finance, Planning and Development, Energy, Mineral Resources, Industry and Trade, and Tourism; the Governor of the Bank of Mozambique (BM); other senior government officials, and representatives of the private sector, civil society, and the donor community.

- The Executive Board approved a three-year PSI arrangement on June 18, 2007 and concluded the third review on January 9, 2009. The fourth review evaluates performance through end-December 2008. All the quantitative assessment criteria (ACs) were met. Implementation of the two end-March 2009 structural benchmarks were delayed.

- In the attached Letter of Intent (LOI) and Memorandum of Economic and Financial Policies (MEFP), the authorities review program performance, outline their policies for the remainder of 2009, and request a one-year arrangement under the High Access Component of the Exogenous Shocks Facility (ESF).

- Since taking office in 2005, the government has demonstrated a firm commitment to reform. Provincial, parliamentary, and presidential elections are scheduled for October 28, 2009. 
Executive Summary

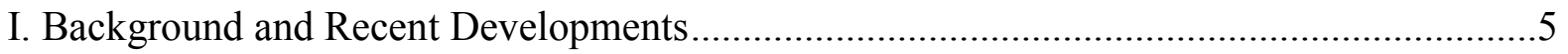

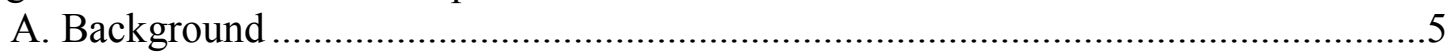

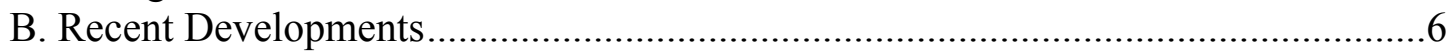

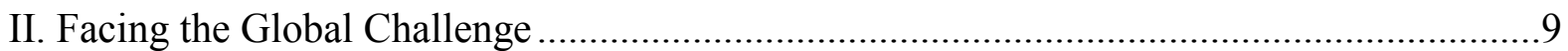

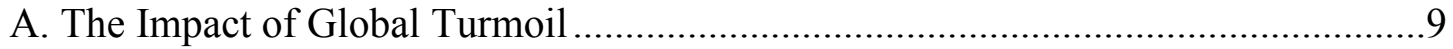

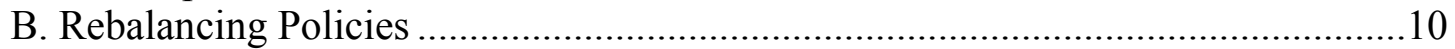

C. Sustaining Momentum for Growth ................................................................. 11

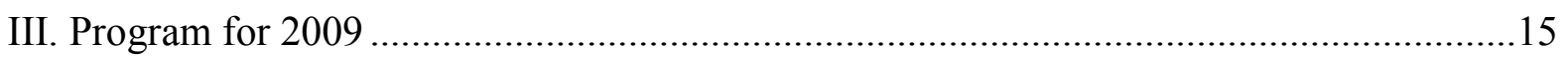

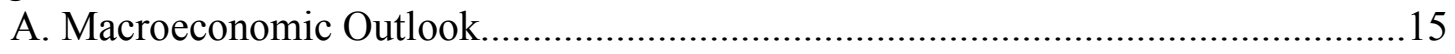

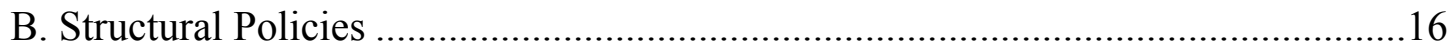

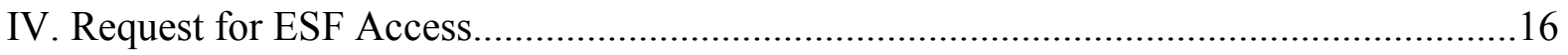

V. Debt Sustainability and Capacity to Repay the Fund ............................................... 17

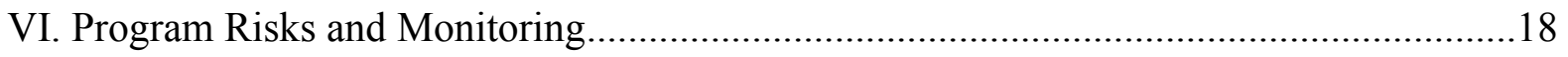

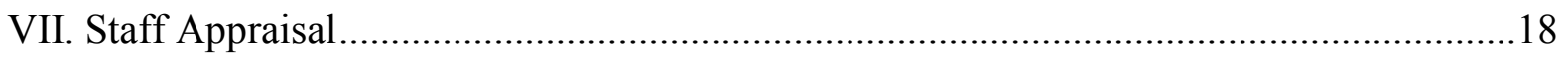

Tables

1. Selected Economic and Financial Indicators, 2007-11 ..............................................20

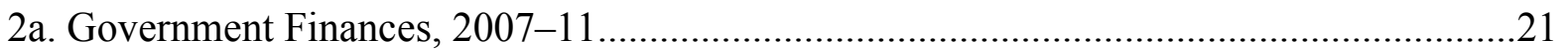

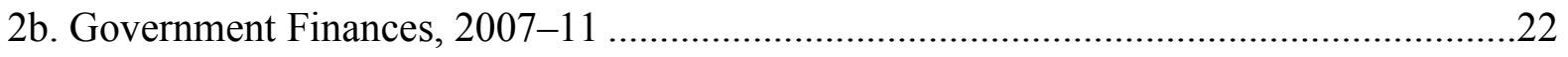

2c. Government Finances, Quarterly, 2008, 2009 ..............................................................23

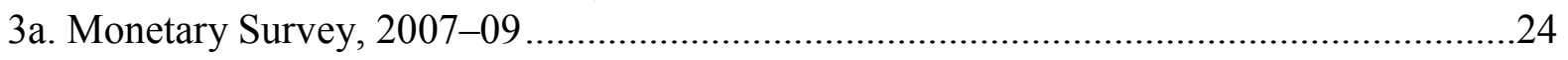

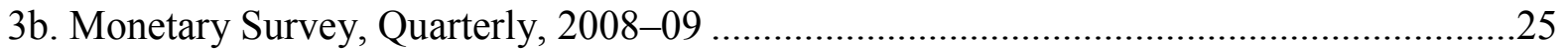

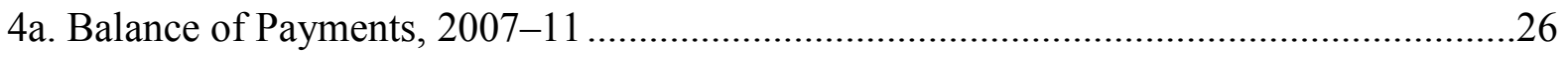

4b. Balance of Payments, 2007-10 ..................................................................................27

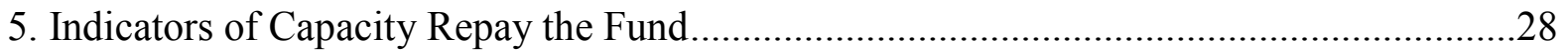

6. Schedule of Disbursements Under the 12-Month ESF Arrangement ...............................29

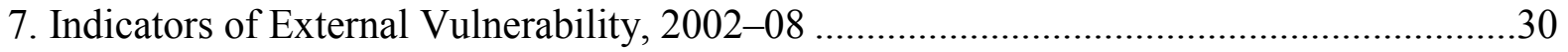

8. Financial Soundness Indicators for Banking Sector, 2001-08 .......................................31

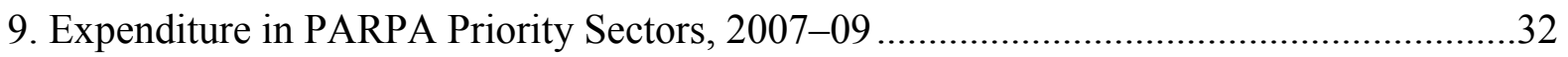

10. Millennium Development Goals, 1990-2007 ............................................................33

Boxes

1. Impact of Lower Aluminum Exports Price...............................................................

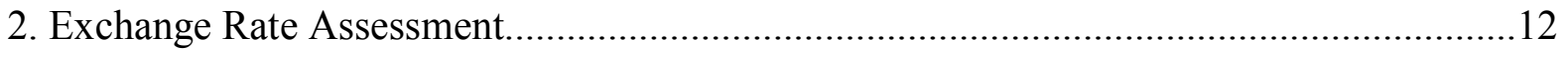

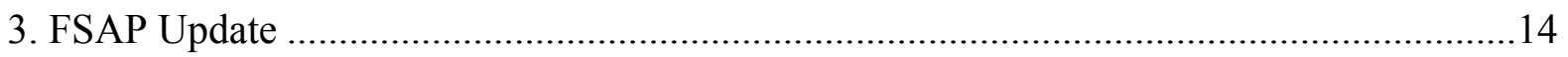




\section{Appendices}

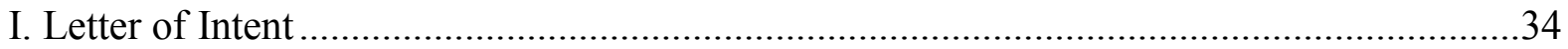

Attachment I. Memorandum of Economic and Financial Policies for 2009 ......................36

Attachment II. Technical Memorandum of Understanding ........................................48

II. Debt Sustainability Update.................................................................................... 54
Annexes
I. Relations with the Fund
.58
II. World Bank-IMF Joint Management Plan ........................................664

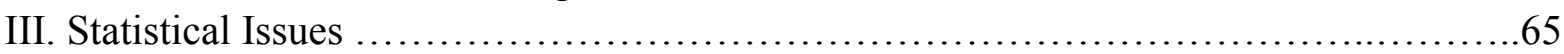




\section{EXECUTIVE SUMMARY}

Mozambique's macroeconomic performance during 2008 was strong. Real GDP growth was 6.8 percent, 12 -month inflation fell to 6.2 percent, U.S. dollar reserves rose, although slipping to 4.4 months of imports, and private sector credit expanded 46 percent. The currency appreciated 24 percent in real effective terms.

Mozambique met all the program quantitative ACs through end-December 2008. Net credit to government fell by 2 percent of GDP, largely because of an advance disbursement of 2009 World Bank budget support, but also because of strong domestic revenues. The BM also comfortably met its targets for reserve money and net international reserves.

Implementation of the two end-March 2009 structural benchmarks has been modestly delayed.

The impact of the global turmoil on Mozambique has so far been contained, but there are significant uncertainties. Mozambique is vulnerable because of its heavy reliance on commodity exports and capital inflows. Although the banking sector is largely insulated, it is vulnerable to credit risk from the economic slowdown and from high credit concentration.

Amid considerable uncertainties about the impact of the global turmoil, Mozambique's macroeconomic prospects for 2009 have weakened. Projected real GDP growth has been reduced to 4.3 percent because of fewer exports and capital inflows. Inflation will remain around 6 percent. Fiscal policy has been eased to maintain priority spending in the face of lower domestic revenues. The program also incorporates a larger monetary expansion to limit the crowding out of credit to the private sector. The external overall balance is expected to deteriorate significantly and external reserves are expected to fall by more than US\$200 million to below 4 months of imports. The authorities are accordingly proposing modifications to the end-June 2009 ACs.

Mozambique is making steady progress on implementing structural reforms. Measures under the program are focused on strengthening macroeconomic policy implementation and improving the business environment.

The policy discussions focused on ways to mitigate the adverse impact of the global economic slowdown on Mozambique's medium-term prospects. Growth over the past decade has been supported by capital inflows and export growth that will be difficult to sustain. The authorities and staff agreed that, if Mozambique's growth momentum is to be maintained, growth will need to be diversified and the government will need to accelerate its reform agenda to improve the business environment, with particular attention to financial sector reform and boosting competitiveness.

The authorities generally agreed with the staff's assessment that the currency is overvalued in real effective terms. Their commitment to pay closer attention to a broader basket of currencies will help redress the overvaluation.

Staff recommend completion of the fourth PSI review and approval of the authorities' request for a 12-month arrangement under the High-Access Component of the ESF with access of 100 percent of quota (SDR 113.6 million). 


\section{BACKGROUND AND RECENT DEVELOPMENTS}

\section{A. Background}

1. Mozambique has had a continuous record of strong macroeconomic performance since the mid-1990s, and its performance compares well with other countries in the region. Output growth over the past decade averaged almost 8 percent. Growth has been sustained by investment financed by large aid and private capital inflows, mainly into the natural resource sector, that together averaged about 24 percent of GDP. In recent years, these flows contributed to an appreciation of the currency in real effective terms. Growth has also been sustained by a strong trade sector- the share of merchandise exports in GDP more than tripled, to nearly 30 percent of GDP, eliminating a trade deficit of about 30 percent of GDP. However, even before the current global crisis, growth had been weakening, with growth projections about 1 percentage point lower than in the past decade. Growth, however, has also been unevenly distributed and, although the national poverty headcount fell from 69 percent in 1997 to 54 percent in 2003, the 2007 assessment suggests that poverty may have increased in recent years, particularly in rural areas - though this may have reflected the impact of the 2005 drought and the surge in fuel and food prices in 2007-08.
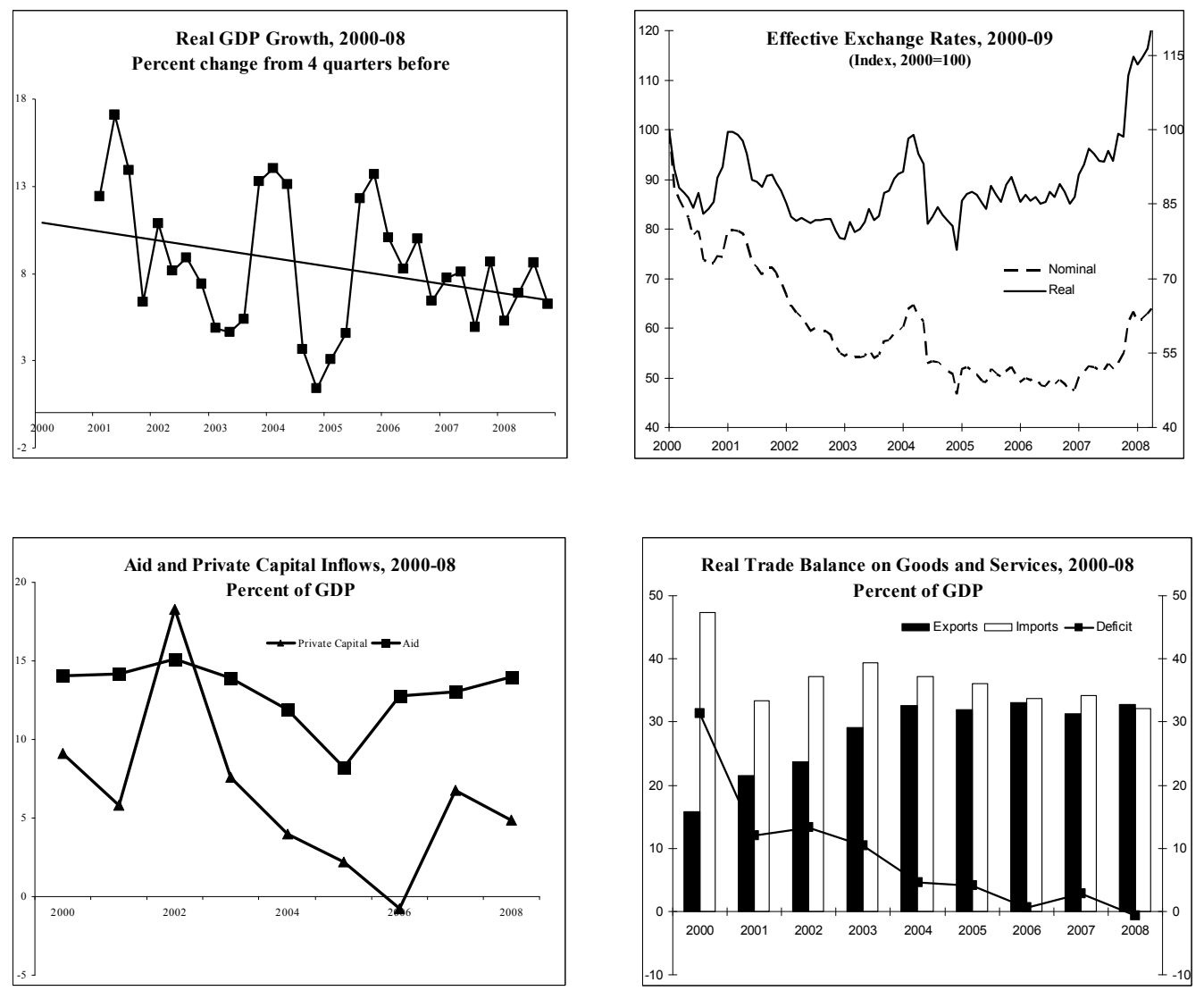


\begin{tabular}{|c|c|c|c|c|c|c|}
\hline \multicolumn{7}{|c|}{ Mozambique: Comparison with Sub-Saharan Africa, Selected Indicators, 2000-08 } \\
\hline & \multicolumn{2}{|c|}{ Mozambique } & \multicolumn{2}{|c|}{ SSA } & \multicolumn{2}{|c|}{ A ex. S.Afr \& Nigeria } \\
\hline & $2000-08$ & 2008 & $2000-08$ & 2008 & $2000-08$ & 2008 \\
\hline Real GDP, percent change & 7.5 & 6.8 & 5.9 & 5.4 & 6.0 & 7.0 \\
\hline $\mathrm{CPI}$ period average, percent change & 12.9 & 10.3 & 10.5 & 11.6 & 12.9 & 11.7 \\
\hline REER, percent change & 10.8 & 24.3 & -3.6 & -2.6 & 2.1 & 6.8 \\
\hline Current account balance (before grants), percent of GDP & -19.4 & -20.4 & -1.2 & -1.3 & -4.7 & -2.8 \\
\hline Broad money, percent of GDP & 29.0 & 33.7 & 41.9 & 48.2 & 26.3 & 31.8 \\
\hline Gross international reserves, months of imports & 5.0 & 4.4 & 4.6 & 5.4 & 4.5 & 4.8 \\
\hline Credit to the private sector, percent of broad money & 52.0 & 55.6 & 75.3 & 74.8 & 51.9 & 54.2 \\
\hline
\end{tabular}

Source: Country authorities and Fund staff estimates.

\section{The previous Article IV consultation in 2007 focused on consolidating} macroeconomic stability and accelerating second-wave reforms to sustain broad-based growth and further reduce poverty. Discussions included strengthening policy implementation, the business environment, and management of the country's natural resources. The government has made significant strides in these areas: it has further consolidated macroeconomic stability despite large external and natural domestic shocks; it has implemented a wide range of measures to improve governance and to reduce the cost of doing business, and has initiated steps to improve natural resource management (including a request for adherence to the EITI and new fiscal laws for the sector).

\section{B. Recent Developments}

3. Macroeconomic performance in 2008 was satisfactory. Real GDP rose 6.8 percent, somewhat exceeding expectations and driven by investment spending supported by high public and private capital inflows, which were equivalent in gross terms to about 23 percent of GDP. Twelve-month inflation fell faster than expected, from a peak of 13 percent in February 2008 to 3 percent in May 2009, mainly because of the sharp drop in international import prices at the end of the year. This permitted the BM to reduce its policy interest rates in February, pushing T-bill rates sharply down.

4. Macroeconomic policy was generally implemented as envisaged (see MEFP Tables 1 and 2). ${ }^{1}$ Mozambique met the three year-end quantitative ACs as well as the revised framework projections established during the $3^{\text {rd }}$ PSI review. The two structural benchmarks for end-March 2009 are expected to be met in July (see MEFP $\mid 9$ and $\uparrow 19$ ).

\footnotetext{
${ }^{1}$ The end-2008 framework projections for net credit to government and net international reserves were revised during the $3^{\text {rd }}$ PSI review, but the ACs remained unchanged from the $2^{\text {nd }}$ review. The end-2008 AC for reserve money was revised during the $3^{\text {rd }}$ review.
} 
Mozambique: Selected Economic Indicators, 2007-11

\begin{tabular}{lrrrrr}
\hline & 2007 & 2008 & 2009 & 2010 & 2011 \\
& Act. & Act. & Proj. & Proj. & Proj. \\
\hline Real GDP, percent change & 7.0 & 6.8 & 4.3 & 5.0 & 6.0 \\
CPI, per avg., percent change & 8.2 & 10.3 & 6.1 & 5.6 & 5.0 \\
CPI, eop., percent change & 10.3 & 6.2 & 5.8 & 5.7 & 5.4 \\
REER, percent change & 6.5 & 24.3 & $\ldots$ & $\ldots$ & $\ldots$ \\
Current account balance (before grants), percent of GDP & -19.4 & -20.4 & -22.9 & -23.2 & -23.3 \\
Gross external reserves coverage, months of imports & 4.7 & 4.4 & 3.9 & 3.6 & 3.4 \\
\hline
\end{tabular}

Sources: Mozambican authorities; and IMF staff estimates and projections.

5. Fiscal policy implementation in 2008 exceeded expectations. ${ }^{2}$ The $\mathrm{AC}$ and the revised projection for net credit to government were met comfortably. As expected, the budget benefited from a large advance disbursement of World Bank 2009 budget support. In addition, domestic revenues were higher and recurrent spending on goods and services were lower than envisaged. Spending execution was strong and priority expenditures were 64 percent of total expenditures. However, as in 2007, there was a significant shortfall in project aid disbursements.

6. The BM met its year-end AC for reserve money. This had been increased during the $3^{\text {rd }}$ PSI review to accommodate the surge in international import prices. However, in the outturn, lower inflation, good management of the seasonal year-end surge in currency demand, and strong fiscal performance enabled the BM to contain reserve money growth in 2008 to below 8 percent. The monetary program was successful in supporting expansion of credit to the private sector, that increased 46 percent during 2008 to almost 19 percent of GDP and 64 percent of deposits. This partly reflected a large increase in foreign currency borrowing as local businesses found it more difficult to access financing overseas (a ban on bank foreign currency lending to unhedged borrowers mitigates exchange rate related credit risk).

\section{The external overall balance was in line with revised projections, and the year-} end $\mathrm{AC}$ on net international reserves was met comfortably. The current account balance was adversely affected by higher import expenditures because of higher international prices. However, this was more than offset by high capital inflows, including higher aid disbursements, so that gross external reserves increased about US\$140 million (but fell to 4.4 months of imports) and the currency appreciated 24 percent in real effective terms. ${ }^{3}$ However, a significant portion of this was reversed in the first four months of 2009.

\footnotetext{
${ }^{2}$ The fiscal framework was revised in mid-2008 to incorporate the advanced disbursement of World bank 2009 budget support and temporary measures to mitigate the social impact of higher fuel and food prices. The government introduced an urban transportation subsidy in February 2008 and suspended fuel-related taxes until 2009 in July. It also launched its Food Production Action Plan. These measures were largely financed by nonpriority spending cuts and additional World Bank budget support.

${ }^{3}$ The BM estimates, using different weights, that the real effective exchange rate in 2008 increased 11 percent.
} 

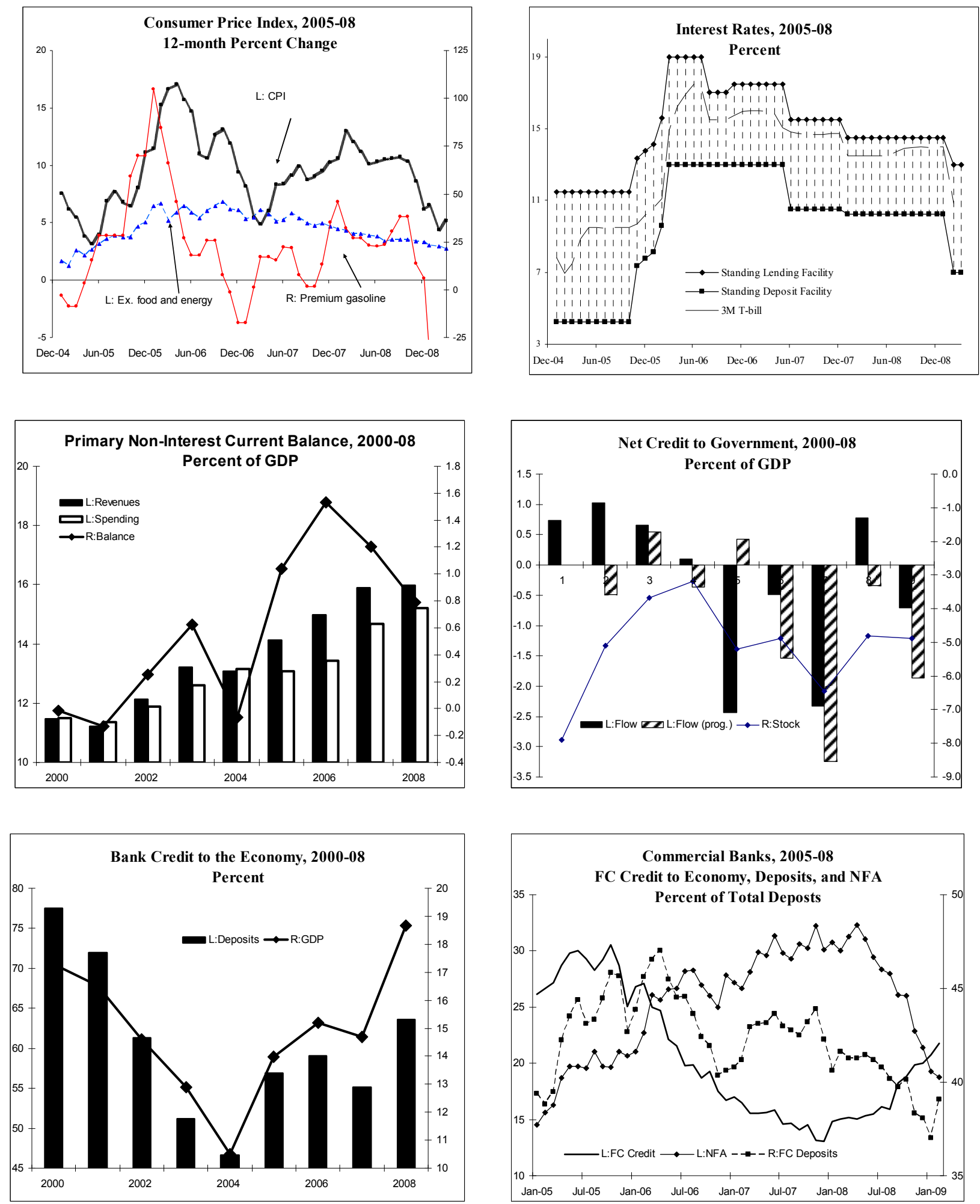


\section{Facing the Global Challenge}

\section{A. The Impact of Global Turmoil}

\section{The impact of the global financial crisis and economic slowdown on Mozambique} has so far been contained and limited to real sector channels, but there are significant uncertainties. Mozambique's economic prospects for 2009 have deteriorated: both its growth and its external balance are likely to be adversely affected by lower exports and private capital inflows than in 2008, and by a less optimistic outlook for project aid.

- Export receipts are projected to decline about 30 percent from their 2008 level due to falling global demand and commodity prices. World prices for aluminum, which accounts for nearly half of total export receipts, have fallen by about 50 percent. Traditional exports are also vulnerable to the weak global environment as well as to the appreciation of the currency in real effective terms.

- Private capital inflows, which have become increasingly important in Mozambique, are contracting as some investors scale back or postpone investment projects because of weaker global demand, depressed commodity prices, and tighter global credit constraints. Private capital inflows are cautiously projected to fall about 40 percent from their 2008 level.

- Donors have indicated that they will meet their aid commitments for 2009, especially for program aid, but receipts are sensitive to the increased exchange rate volatility. Moreover, project aid disbursements are already being delayed and may fall further short of commitments as fiscal pressures mount in donor countries. Consequently, while aid flows are expected to be higher in 2009 than in 2008, the magnitude of the scaling-up of aid has been pulled back from what was previously projected.

- Mozambique's macroeconomic prospects are also likely to be adversely affected by the spill-over effects of the projected economic contraction in South Africa.

\section{Box 1. Mozambique: Impact of Lower Prices for Aluminum Exports}

The aluminum smelter Mozal provides about half of Mozambique's export receipts and 70 percent of the manufacturing sector's output. In 2008, despite all-time high aluminum prices, production fell by 10 percent due to constraints on power supplied via the South Africa energy supplier ESKOM.

Mozal's production and sales volumes in the first quarter of 2009 remained comparable with levels in 2008, despite the plunge in global demand and prices for aluminum. The market for Mozal's high quality aluminum has suffered less from the global downturn than the rest of the aluminum market. Moreover, the high fixed costs typical of the industry are conducive to smoothing output in response to short-term fluctuations in market conditions, and the parent company, BHP Billiton, has opted to maintain Mozal's production levels. Nevertheless, as sales receipts have plunged, Mozal has cut operating costs substantially, especially spending on local expenditures and employment. 
9. A recent FSAP Update mission found that the exposure of Mozambique's banking sector to the turmoil in global financial system is limited (see Box 3). However, the sector is vulnerable to credit risk arising from the economic slowdown and from high credit concentration. In this context, reinforcing the loan classification rules is particularly important. Staff and the authorities agreed that it would be important for the BM to more closely monitor key financial indicators and improve cooperation with foreign supervisors.

10. Against a backdrop of a highly uncertain global economic environment, Mozambique's macroeconomic prospects remain positive, but have weakened. Its growth in 2009 could fall several percentage points below trend. In view of WEO projections for a slow global recovery, growth is projected to strengthen in 2010, but will probably remain below 6 percent until 2011. Similarly, the external overall balance is projected to turn negative in both 2009 and 2010; turning positive again in 2011 and stabilizing external reserves at about 3.4 months of imports. The authorities acknowledged the weakened growth prospects. They noted that the growth projections are cautious and emphasized their intention to make every effort to sustain growth. Staff agreed that Mozambique's prudent macroeconomic policies in recent years will help the country weather the global turmoil.

\section{B. Rebalancing Policies}

11. The government is committed to its medium-term development strategy. ${ }^{4}$ Under the strategy and the PSI-supported program, fiscal policy has avoided domestic financing to make room for private sector credit and monetary policy has been geared to reducing inflation to 5-6 percent in the context of a flexible exchange rate regime and an external reserve target of 4-5 months of imports. ${ }^{5}$

12. The authorities and staff agreed that the overall macroeconomic strategy remains appropriate, but that the global turmoil warrants some rebalancing. In the near term, the economic slowdown warrants a temporary easing of fiscal policy and the use of domestic budget financing to maintain spending in the face of lower domestic revenues. Despite spending pressures in the run-up to national elections in October, the authorities stated their intention to adhere strictly to budgeted expenditures and to protect priority spending. The low public domestic debt ( 8 percent of GDP) and interest costs (about 0.3 percent of GDP) provide the fiscal space for additional domestic financing. Under the medium-term strategy, this would be subsequently repaid. The authorities and staff also acknowledged the scope for easing monetary policy to accommodate higher domestic budget financing and to limit the crowding out of private sector credit.

\footnotetext{
${ }^{4}$ As outlined in the Action Plan for Reducing Absolute Poverty (PARPA), the Mozambican Poverty Reduction Strategy for 2005-09.

${ }^{5}$ The Fund's 2008 Annual Report on Exchange Arrangements and Exchange Restrictions (AREAER) classified the exchange arrangement in Mozambique as "managed floating with no predetermined path for the exchange rate," while the revised classification methodology (effective February 1, 2009) characterizes it as floating.
} 
13. The weaker prospects for the global economy may also require a rebalancing in the medium term to sustain Mozambique's growth momentum. Mozambique's weakened growth prospects will exert pressure on the authorities to maintain eased macroeconomic policies in 2010, including domestic budget financing, before restoring their medium-term macroeconomic strategy in 2011 and beyond. However, in view of the WEO projections for a slow global recovery, the time frame for restoring Mozambique's high levels of private capital inflows and export growth and the risks to the projected scaling-up of aid will pose challenges for the authorities' ambitious growth objectives.

\begin{tabular}{|c|c|c|c|c|c|c|c|c|c|c|c|c|}
\hline \multicolumn{13}{|c|}{ Mozambique: Private Capital and Aid Inflows, 2000-11, percent of GDP ${ }^{1}$} \\
\hline & 2000 & 2001 & 2002 & 2003 & 2004 & 2005 & 2006 & 2007 & 2008 & 2009 & 2010 & 2011 \\
\hline & Act. & Act. & Act. & Act. & Act. & Act. & Act. & Act. & Act. & Proj. & Proj. & Proj. \\
\hline Private capital inflows & 9.1 & 5.8 & 18.3 & 7.6 & 4.0 & 2.2 & -0.8 & 6.8 & 4.9 & 3.3 & 3.1 & 3.0 \\
\hline Aid & 14.1 & 14.2 & 15.1 & 13.9 & 11.9 & 8.2 & 12.8 & 13.0 & 14.0 & 15.0 & 17.3 & 19.8 \\
\hline Net capital inflows & 23.2 & 20.0 & 33.5 & 21.5 & 15.9 & 10.4 & 12.0 & 19.8 & 18.9 & 18.3 & 20.4 & 22.8 \\
\hline
\end{tabular}

\section{Sustaining Momentum for Growth}

14. To sustain momentum for growth, the authorities and staff agreed on the need to further consolidate macroeconomic stability and to continue attracting capital inflows. However, it was also recognized that the sources of growth need to be diversified and that traditional exports and domestic sectors, especially agriculture, need to play a larger part. To this end, the authorities are committed to sustaining and, to the extent possible, accelerating the reform agenda to improve the business environment, with particular attention to Mozambique's competitiveness, encouraging domestic as well as foreign investment, and strengthening financial intermediation. They also intend to enhance the reforms introduced in 2008 to boost agriculture in response to the rise in world food prices. They are therefore revising the current development strategy that expires this year. The revised strategy will place greater emphasis on rural development (especially agriculture), the tourism sector, and building a skilled labor force.

\section{Boosting competitiveness}

15. Boosting Mozambique's competitiveness is a major priority. The currency appreciated by 24 percent in real effective terms in 2008. That appreciation reflected heightened volatility in exchange rates for the major currencies and sales by the BM, which had the effect of stabilizing the exchange rate versus the US dollar, thus leading to a marked appreciation against South Africa, Mozambique's principal regional trading partner. Staff estimates (see Box 2), while subject to considerable uncertainty, indicate a significant overvaluation. However, the currency depreciated about 6 percent against the US dollar during the first four months of 2009, reversing about a third of the real appreciation in 2008. The authorities generally agreed with the staff's assessment. 


\section{Encouraging investment}

\section{Reducing the costs of doing business in Mozambique is essential to encourage} domestic and foreign investment and improve competitiveness. Recent surveys continue to identify limited access to credit, poor infrastructure, burdensome regulations, and a shortage of skilled labor as impediments to business activity. Staff stressed growing private sector concerns over labor market restrictions. The government has a reform strategy in place, but progress in relation to Mozambique's main trading partners has been slow. The authorities recognize that this area requires continued emphasis.

18. Mozambique's natural resources have attracted significant foreign private capital. The country will need to accelerate its agenda to improve public management of these resources to encourage further capital inflows and ensure that the benefits pass to the rest of the economy. The authorities and staff agreed that it would be important to implement rigorously the recently approved fiscal regime for megaprojects and for Mozambique to adhere to and implement the principles of the EITI as quickly as possible. Moreover, the global turmoil has highlighted the need to reinforce the authorities' capacity to monitor and assess the budgetary and macroeconomic implications of megaprojects.

\section{Strengthening financial intermediation}

19. Weak financial intermediation and access to credit are a major impediment to private sector development (see Box 3). The vast majority of Mozambicans are still not served by registered financial institutions and deposit and credit ratios relative to GDP are low. The key ratios improved in 2008 and at the end of 2008, broad money reached 34 percent of GDP, credit to the private sector reached about 19 percent of GDP and about 56 percent of broad money. These ratios are well below the average for sub-Saharan Africa. ${ }^{6}$

\section{The country's poor performance on access and outreach indicators can be} attributed to economy-wide structural impediments and the lack of competition in the banking sector. Improving financial intermediation is a long, slow and incremental process. In addition to their commitment to further macroeconomic consolidation, the authorities have a structural reform agenda in place to support financial deepening. However, improving financial intermediation is a long, slow and incremental process. In this context, the authorities agreed that moving quickly on the findings of the FSAP Update would help strengthen Mozambique's financial sector.

\footnotetext{
${ }^{6}$ The ratios are more broadly aligned to the regional averages, excluding South Africa and Nigeria.
} 


\section{Box 3. Mozambique: FSAP Update}

The recent FSAP Update mission in February 2009 found that Mozambique has made significant progress toward developing and strengthening the financial sector and improving central bank operations since the 2003 FSAP. Most of the 2003 recommendations have been implemented. The authorities strengthened bank supervisory powers; introduced International Financial Reporting Standards (IFRS) for banks; and laid the foundations for risk-based supervision. Bank soundness was improved by restructuring of problem banks and cleaning up of balance sheets. Enhanced communication and increasing use of market instruments have improved monetary and exchange rate policy implementation, and the level and volatility of real interest rates have declined.

However, the FSAP Update also found limited progress in financial deepening. The vast majority of Mozambicans are still not served by registered financial institutions and deposit and credit ratios relative to GDP are low in both absolute terms and in comparison to similar countries. The poor performance on access and outreach indicators can be attributed to two sets of factors. First, there are economy-wide, structural impediments to financial intermediation that limit the number of credit-worthy clients or increase the costs and risks of offering financial services. Second, lack of competition in the banking sector, high bank overhead costs and interest spreads, and high bank commissions and fees push up borrowing costs. Improving financial intermediation is a long, slow and incremental process

The FSAP Update also found that the banking sector's exposure to global financial turmoil is limited because the sector is not highly integrated into global financial markets, which means direct contagion risks are few. The only possible exception is the risk arising from the high concentration of subsidiaries' foreign assets in correspondent accounts with their parent banks: in case of major difficulties for the parents, these assets could be impaired. Given the strength of parent banks, however, this risk is very limited. The major source of risk in the system is credit risk arising from the economic slowdown and the relatively high concentration of loan portfolios in a few sectors.

The FSAP Update made recommendations concerning access to finance; banking supervision; money, debt markets and liquidity management; the non-bank financial sector (pension and insurance); and the payments system. It also made recommendations for responses to the global financial crisis to strengthen risk monitoring, home-host cooperation on banking supervision, and crisis management arrangements.

The BM is committed to moving ahead on the findings of the FSAP Update. It will assess and finalize an action plan to implement the FSAP recommendations and, by endDecember 2009, form a task force to monitor implementation. Meanwhile, in line with these recommendations, the BM will issue new regulations to redefine the loan classification rules (end-September 2009 structural benchmark). It is preparing improvements in its remedial action framework for banks in difficulty and is strengthening its crisis preparedness and contingency planning. 


\section{Program FOR 2009}

\section{A. Macroeconomic Outlook}

21. Mozambique's macroeconomic prospects for 2009 remain positive, but have weakened because of the global economic slowdown. Projected real GDP growth has been cautiously reduced to 4.3 percent, down from 6.8 percent in 2008 and more than 3 percentage points below the average for the past decade. Inflation at the end of 2009 is expected to remain around 6 percent because of modest increases in international fuel and food prices and the recent currency depreciation.

\section{Easing fiscal policy}

22. The fiscal program for $\mathbf{2 0 0 9}$ has been revised: the government now expects to incur domestic financing of about 1.8 percent of GDP, about 1.1 percent of GDP higher than previously envisaged. Most of the increase arises from the adverse impact of the global economic crisis on domestic revenues, which are expected to be about 0.8 percent of GDP lower than envisaged. As a result, despite the reinstatement of fuel-related taxes suspended in the second half of 2008 and the continued increase in the domestic revenue effort of about 0.5 percent of GDP, the domestic revenue share of GDP is expected to be lower in 2009 than in 2008. In other respects, the fiscal program for 2009 is largely as previously envisaged. Domestically-financed spending will remain within budget allocations, though spending relative to a lower GDP will now be higher than intended. In line with shortfalls in 2007 and 2008 and with delays already observed in early 2009, the outlook for project aid is now less optimistic than envisaged.

\section{Monetary policy accommodates}

23. Monetary policy will remain geared towards keeping inflation low, with reserve money as the operational target. The BM is committed to a floating exchange rate, while monitoring a broad currency basket that reflects Mozambique's trading patterns to guide reserve sales to the market. Reserve money growth for 2009 has been increased to 12.5 percent to partially accommodate the expansion in domestic budget financing and to limit the crowding out of private sector credit. Any resulting inflationary pressures are likely to be manageable as inflation is already low and external inflationary pressures appear weak. The monetary program makes room for private sector credit to expand nearly 30 percent.

\section{The external balance deteriorates}

24. The external shock will cause the balance of payments to deteriorate. The overall balance of payments in 2009 is projected to deteriorate by US\$382 million compared to 2008 as the trade deficit widens, foreign direct investment contracts, and foreign borrowing by the private sector is curtailed. As a result, gross international reserves are expected to fall by US\$221 million in 2009 to 3.9 months of imports - well below the average of the past decade 
and below the BM's medium-term objective of 4 to 5 months import cover. Mitigating factors include much lower projected fuel imports and dividend payments by megaprojects. A projected decline in exports of US $\$ 777$ million primarily reflects a 53 percent fall in the price of aluminum. It also reflects a reduction in trading partner demand for traditional exports and service receipts from tourism and remittances. Foreign direct investment is projected to fall US\$67 million, and foreign borrowing by the private sector to fall US\$37 million.

\section{B. Structural Policies}

\section{The government is making steady progress on the structural reform agenda} detailed in the MEFP. In improving macroeconomic policy operations, the government is bolstering its domestic revenue effort and public financial management. It is also reforming the civil service salary and pension policy and the social security system. The BM is strengthening its monetary policy framework and adapting monetary policy to better reflect the determinants of price stability. As noted, it is also committed to moving ahead on the findings of the FSAP Update.

26. Improving the business environment is a priority. With the help of development partners, the government is implementing reforms to support private sector development, natural resource management, and public sector governance. The EITI Board approved Mozambique as a candidate for adherence to the EITI on May 18, 2009. Initiatives are underway to improve public sector governance, and the government is working on a public enterprise law, a public-private partnership law, and a decentralization strategy.

\section{The recently approved new foreign exchange law liberalizes current account} transactions. In the course of 2009, the government expects to officially inform the Fund of its acceptance of the obligations under Article VIII sections 2, 3, and 4 of the Fund's Articles of Agreement.

\section{REQUEST FOR ESF ACCESS}

28. The scope for supportive macroeconomic policies to offset the impact of the global economic slowdown is curtailed by the large projected decline in external reserves. Staff estimate that most of the deterioration in the external overall balance is the direct result of the external shock; the fiscal expansion is estimated to account for only about US\$42 million of the reserve loss in 2009 through higher import demand. ${ }^{7}$ The authorities view maintaining reserves at 4-5 months of imports to be desirable given Mozambique's high exposure to potentially volatile private capital and income flows, and its high degree of dollarization. Maintaining reserve coverage in 2009 at 4.5 months of imports would imply a

\footnotetext{
${ }^{7}$ This estimate assumes a fiscal multiplier of 0.5 for tax measures and a unit income elasticity of nonmegaproject import demand.
} 
balance of payments need of about US\$210 million. As the risks surrounding the balance of payments projection are skewed downwards, bolstering reserves will provide the necessary room for maneuver should the downside materialize.

\begin{tabular}{|c|c|}
\hline \multicolumn{2}{|l|}{ (Millions of U.S. dollars) } \\
\hline & 2009 \\
\hline Direct impact of the external shock & -252 \\
\hline Megaproject exports & -659 \\
\hline Traditional exports & -118 \\
\hline Service exports & -42 \\
\hline Oil imports & 206 \\
\hline Dividend payments & 623 \\
\hline Foreign direct investment & -67 \\
\hline Foreign borrowing by the private sector & -37 \\
\hline Short term capital & -157 \\
\hline Fiscal stimulus & -42 \\
\hline Other & -88 \\
\hline Change in overall balance of payments (-ve, deterioration) & -382 \\
\hline
\end{tabular}

29. Staff believe that Mozambique is eligible for a one-year ESF arrangement with access of 100 percent of quota (SDR 113.6 million) to be made available in three drawings following completion of the fourth, fifth and sixth PSI reviews. In staff's assessment, Mozambique faces balance of payments difficulties caused primarily by a sudden and exogenous shock. Moreover, the shock is expected to be temporary, as the overall balance is projected to revert to a surplus in 2011. Access to the ESF would provide timely coverage of about two-thirds of the direct balance of payments impact of the external shock or a somewhat larger portion of the balance of payments need. Moreover, the use of Fund resources should catalyze support from donors that are considering stepping up their assistance, notably the World Bank. In staff's view, the relatively high access is also justified by Mozambique's strong track record under the PSI arrangement and by the authorities' continued commitment to sound policies. The government is committed to a safeguards assessment to be completed by the time of the first review under the ESF arrangement.

\section{Debt Sustainability and CAPaCity to Repay the Fund}

\section{Mozambique's risk of debt distress remains low, though its public debt} sustainability ratios will deteriorate (see Appendix II). The larger part of Mozambique's public debt is external debt. External debt and debt service are projected to rise relative to GDP, exports and revenues during 2009 and beyond mostly because of lower nominal GDP, export receipts, and revenues measured in US dollars. The use of Fund resources will have 
only a modest impact on the ratios and the debt indicators will continue to remain well below their thresholds. In staff's view, Mozambique has adequate capacity to repay the Fund.

\section{Program Risks and Monitoring}

31. The major program risks arise from the considerable uncertainties surrounding the impact of the global financial turmoil and economic slowdown. There are large risks surrounding the impact on exports and foreign capital inflows, and domestic fiscal pressures may oblige donors to revisit their aid commitments. The BM will need to be vigilant for risks in the financial sector, though these currently appear to be contained. On the domestic front, the government will need to resist unrealistic spending pressures during an election year.

32. The revised program modifies the three quantitative assessment criteria for endJune 2009. ACs for end-December 2009 are proposed in Table 1 of the MEFP. Structural benchmarks are shown in Table 2 of the MEFP. The end-June and end-December 2009 ACs, as well as the continuous ACs, will also be performance criteria under the ESF arrangement.

\section{StAFF ApPraisal}

33. Mozambique's macroeconomic performance during 2008 has continued to be strong. Real GDP growth was resilient to challenges from the external environment and inflation has fallen faster than expected. All the quantitative assessment criteria through endDecember 2008 were met. Staff support the authorities' proposed modifications of the endJune 2009 assessment criteria.

34. The government has demonstrated its ability to mitigate the impact of internal and external shocks with flexible policy responses. The government reacted in an effective and timely manner to higher fuel and food import prices in 2008 with an appropriate mix of fiscal and monetary responses.

35. Macroeconomic prospects have now been weakened by the global economic slowdown and there remain many uncertainties and risks. Growth is expected to slow markedly and there is expected to be a significant adverse impact on the balance of payments and on reserves. The authorities will need to monitor international and domestic developments closely and stand ready to respond if necessary. Their readiness to consult closely with Fund staff on possible measures is welcome. Staff believe that the authorities' request for access to the ESF is justified and appropriate.

36. Staff agree with the authorities' strategy of easing fiscal and monetary policy in the near term. Staff also agree that the easing should be seen in the context of Mozambique's prudent medium-term macroeconomic strategy that remains broadly appropriate. The authorities' commitment to expenditure restraint during an election year is particularly welcome. 
37. Mozambique's medium-term macroeconomic prospects have been clouded by the gloomy prospects for the global economy, especially in view of its heavy reliance on exports and capital inflows. To the extent possible, it will be important to diversify the sources of growth and to accelerate structural reforms to boost Mozambique's competitiveness, encourage investment, and improve financial intermediation. The authorities' commitment to moving ahead on the findings of the FSAP Update is welcome.

38. The authorities recognize that the currency is significantly overvalued in real effective terms. Their commitment to pay closer attention to a broader basket of currencies will help redress the overvaluation.

39. The authorities have continued to make noticeable progress in strengthening macroeconomic policy implementation. Staff encourage their efforts to mobilize more domestic revenue and improve public financial management. The steps being taken to strengthen monetary policy formulation and implementation are also welcome. In the current global financial crisis, effective banking supervision is particularly important.

40. The authorities are making progress on other structural reforms to support private sector development, better manage Mozambique's natural resources, and improve public sector governance. Rapid and tangible improvements in the business environment will be essential for sustained high economic growth. The intention to adhere to the EITI is encouraging, but it will be important to continue moving forward in this critical area.

41. The staff welcome the authorities' intention to accept the obligations under Article VIII sections 2, 3, and 4 of the Fund's Articles of Agreement.

42. Based on performance to date and Mozambique's continued commitment to program implementation, staff recommend the completion of the fourth PSI review, and approval for Mozambique's request for a one-year arrangement under the High Access Component of the ESF.

43. It is proposed that the next Article IV consultation be held in accordance with the decision on consultation cycles approved on July 15, 2002. 
Table 1. Mozambique: Selected Economic and Financial Indicators, 2007-11

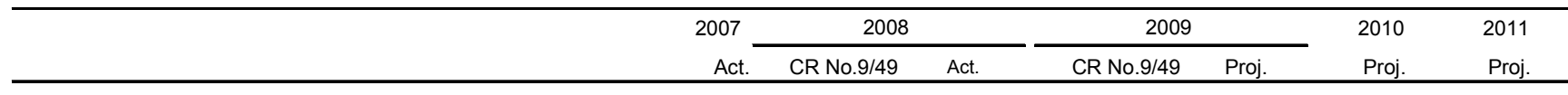

(Annual percentage change, unless otherwise indicated)

National income and prices Nominal GDP (billions of MT)

Nominal GDP growth

Real GDP growth

GDP per capita (U.S. dollars)

Consumer price index (annual average)

Consumer price index (end of period)

External sector

Merchandise exports

Merchandise exports, excluding megaprojects

Merchandise imports

Merchandise imports, excluding megaprojects

Terms of trade

Nominal effective exchange rate (end of period) ${ }^{1}$

Real effective exchange rate (end of period) ${ }^{1}$

$\begin{array}{rrrrrrr}208 & 235 & 239 & 266 & 261 & 288 & 320 \\ 15.4 & 13.4 & 15.0 & 13.4 & 9.1 & 10.2 & 11.3 \\ 7.4 & 6.5 & 6.8 & 6.2 & 4.3 & 5.0 & 6.0 \\ 398 & 469 & 477 & 502 & 440 & 445 & 469 \\ 8.2 & 10.4 & 10.3 & 7.1 & 6.1 & 5.6 & 5.0 \\ 10.3 & 9.2 & 6.2 & 7.6 & 5.8 & 5.7 & 5.4 \\ & & & & & & \\ 1.3 & & & & & & \\ -17.9 & 11.1 & 41.1 & 6.2 & -14.7 & 6.2 & 7.1 \\ 4.8 & 17.5 & 23.0 & 1.1 & -3.5 & -1.8 & 10.4 \\ 4.7 & 20.5 & 26.9 & 1.6 & -11.6 & 14.1 & 8.7 \\ 4.2 & -14.5 & -15.5 & -4.4 & -21.2 & 6.4 & -21.2 \\ 2.2 & \ldots & 22.5 & \ldots & \ldots & \ldots & \ldots \\ 6.5 & \ldots & 24.3 & \ldots & \ldots & \ldots & \ldots\end{array}$

(Annual changes in percent of beginning-of-period stock of money-M3, unless otherwise indicated)

Money and credit ${ }^{2}$

Net foreign assets

Net domestic assets

Of which: net credit to the government ${ }^{3}$ credit to the economy

Broad money-M3 (12-month percent change)

M2 (12-month percent change)

Velocity (GDP/M2)

Interest rate for 90 -day treasury bills (percent; end of period)

11.8
13.4
3.0
6.5
25.2
23.0
4.9
14.8

$\begin{array}{rr}11.6 & 4.0 \\ 8.6 & 16.3 \\ -3.5 & -2.5 \\ 17.1 & 21.1 \\ 20.2 & 20.3 \\ 22.1 & 26.0 \\ 4.5 & 4.4 \\ \ldots & 14.0\end{array}$

$\begin{array}{rr}7.6 & -0.1 \\ 8.5 & 15.1 \\ 2.3 & 5.8 \\ 13.5 & 15.6 \\ 16.1 & 15.0 \\ 15.8 & 15.8 \\ 4.4 & 4.2 \\ \ldots & \ldots\end{array}$

(Percent of GDP)

Investment and saving

Gross domestic investment

Government

Other sectors

Gross domestic savings (excluding grants)

Government

Other sectors

Current account, before grants

Government budget

Total revenue

Total expenditure and net lending

Overall balance, before grants

Total grants

Overall balance, after grants

Domestic primary balance

External financing (incl. debt relief)

Net domestic financing ${ }^{4}$

$\begin{array}{rrr}17.9 & 21.9 & 18.5 \\ 11.7 & 14.9 & 11.6 \\ 6.2 & 7.0 & 6.9 \\ -1.5 & 0.7 & -1.9 \\ 0.6 & -0.2 & 0.3 \\ -2.1 & 0.9 & -2.2 \\ -19.4 & -21.2 & -20.4\end{array}$

23.7
16.3
7.4
3.8
0.5
3.3
-19.8

21.9

15.6

6.3

$-1.1$

$-1.0$

$-0.1$

$-22.9$

$\begin{array}{rr}22.7 & 22.1 \\ 16.8 & 18.8 \\ 5.9 & 3.3 \\ -0.5 & -1.2 \\ -0.6 & -0.2 \\ 0.1 & -1.0 \\ -23.2 & -23.3\end{array}$

$\begin{array}{rrrrrrr}15.9 & 15.8 & 16.0 & 16.9 & 15.7 & 16.0 & 16.5 \\ 28.1 & 32.4 & 27.9 & 34.4 & 33.1 & 34.4 & 36.5 \\ -13.1 & -16.5 & -11.6 & -17.5 & -17.4 & -18.4 & -20.0 \\ 9.3 & 12.0 & 9.5 & 12.1 & 11.8 & 14.0 & 16.1 \\ -3.8 & -4.5 & -2.2 & -5.4 & -5.6 & -4.3 & -3.9 \\ -3.4 & -3.6 & -3.1 & -4.0 & -5.6 & -5.2 & -4.3 \\ 3.6 & 5.6 & 4.0 & 4.3 & 3.4 & 3.5 & 4.2 \\ 0.2 & -1.1 & -1.9 & 0.7 & 1.8 & 0.8 & -0.3\end{array}$

External current account, before grants

Overall balance of payments

Net international reserves (end of period)

Gross international reserves (end of period)

In months of imports of goods and nonfactor services

(Millions of U.S. dollars, unless otherwise indicated)

$\begin{array}{rrrrrrr}-1,576 & -2,058 & -2,021 & -2,107 & -2,135 & -2,229 & -2,408 \\ 136 & 121 & 159 & -17 & -223 & -146 & 60 \\ 1,508 & 1,625 & 1,644 & 1,608 & 1,421 & 1,275 & 1,336 \\ 1,520 & 1,641 & 1,660 & 1,625 & 1,440 & 1,294 & 1,355 \\ 4.7 & 4.6 & 4.4 & 4.5 & 3.9 & 3.6 & 3.4\end{array}$

Sources: Mozambican authorities; and IMF staff estimates and projections

${ }^{1}$ Minus sign indicates depreciation.

${ }^{2}$ Data from 2008 onwards based on data following technical revisions to monetary survey finalized in January 2008.

${ }^{3}$ Includes movement in the government account set abroad with the proceed of the Moatize coal mine concession.

${ }^{4}$ Includes World Bank disbursement of 2009 budget support in 2008. 
Table 2a. Mozambique: Government Finances, 2007-11

Billions of MT

\begin{tabular}{|c|c|c|c|c|c|c|c|}
\hline & \multirow{2}{*}{$\begin{array}{r}2007 \\
\text { Act. }\end{array}$} & \multicolumn{2}{|c|}{2008} & \multicolumn{2}{|c|}{2009} & \multirow{2}{*}{$\begin{array}{r}2010 \\
\text { Proj. }\end{array}$} & \multirow{2}{*}{$\begin{array}{r}2011 \\
\text { Proj. }\end{array}$} \\
\hline & & CR No.9/49 & Act. & CR No.9/49 & Proj. & & \\
\hline Total revenue & 33.06 & 37.20 & 38.27 & 44.98 & 41.09 & 46.13 & 52.95 \\
\hline Tax revenue & 29.08 & 32.57 & 34.02 & 39.06 & 35.39 & 40.52 & 45.82 \\
\hline Nontax revenue & 3.98 & 4.64 & 4.25 & 5.93 & 5.71 & 5.61 & 7.13 \\
\hline Total expenditure and net lending & 58.45 & 76.04 & 66.85 & 91.61 & 86.46 & 98.95 & 117.01 \\
\hline Current expenditure & 31.84 & 37.72 & 37.63 & 43.59 & 43.59 & 47.85 & 53.60 \\
\hline Compensation to employees & 16.09 & 18.86 & 19.27 & 22.46 & 23.32 & 24.19 & 26.33 \\
\hline Goods and services & 8.34 & 9.93 & 9.71 & 11.56 & 10.71 & 12.10 & 13.51 \\
\hline Interest on public debt & 1.28 & 1.31 & 1.26 & 1.39 & 1.39 & 1.61 & 2.49 \\
\hline Transfer payments & 6.13 & 7.62 & 7.40 & 8.18 & 8.18 & 9.95 & 11.27 \\
\hline Domestic current primary balance & 2.50 & 0.79 & 1.89 & 2.78 & -1.11 & -0.11 & 1.84 \\
\hline Capital expenditure & 24.31 & 35.01 & 27.74 & 43.32 & 40.68 & 48.48 & 60.22 \\
\hline Grant finance projects & 6.64 & 11.84 & 6.08 & 13.89 & 8.07 & 11.86 & 15.49 \\
\hline Projet loans & 3.98 & 5.35 & 3.56 & 7.21 & 7.10 & 4.56 & 4.94 \\
\hline Locally financed & 7.94 & 9.51 & 10.09 & 13.45 & 13.45 & 15.07 & 15.97 \\
\hline Grant-financed special programs & 3.70 & 6.55 & 6.36 & 7.54 & 10.67 & 15.50 & 22.24 \\
\hline Direct financing & 0.85 & 0.85 & 0.80 & 1.24 & 1.39 & 1.51 & 1.58 \\
\hline Net lending & 2.30 & 3.31 & 1.48 & 4.70 & 2.18 & 2.61 & 3.20 \\
\hline Lending to public enterprises & 2.48 & 3.48 & 1.61 & 4.74 & 2.23 & 2.84 & 3.50 \\
\hline Locally financed & -0.18 & -0.17 & -0.13 & -0.05 & -0.05 & -0.23 & -0.31 \\
\hline Domestic primary balance, before grants, above the line ${ }^{1}$ & -5.27 & -8.56 & -8.06 & -10.62 & -14.51 & -14.95 & -13.82 \\
\hline Unallocated revenue $(+) /$ expenditure $(-)^{2}$ & -1.78 & 0.00 & 0.75 & 0.00 & 0.00 & 0.00 & 0.00 \\
\hline Overall balance, before grants (below the line) & -27.16 & -38.84 & -27.84 & -46.63 & -45.36 & -52.82 & -64.07 \\
\hline Grants received & 19.27 & 28.16 & 22.64 & 32.21 & 30.84 & 40.41 & 51.61 \\
\hline Project & 11.19 & 19.23 & 13.80 & 22.66 & 20.13 & 28.86 & 39.31 \\
\hline Investment projects & 6.64 & 11.84 & 6.16 & 13.89 & 8.07 & 11.86 & 15.49 \\
\hline Special programs & 3.70 & 6.55 & 6.84 & 7.54 & 10.67 & 15.50 & 22.24 \\
\hline Direct financing & 0.85 & 0.85 & 0.80 & 1.24 & 1.39 & 1.51 & 1.58 \\
\hline Nonproject & 8.08 & 8.92 & 8.84 & 9.55 & 10.71 & 11.55 & 12.30 \\
\hline Overall balance, after grants & -7.90 & -10.69 & -5.20 & -14.42 & -14.52 & -12.41 & -12.46 \\
\hline Net external financing & 7.47 & 13.26 & 9.65 & 11.55 & 8.76 & 10.06 & 13.44 \\
\hline Disbursements & 8.29 & 13.23 & 9.56 & 12.74 & 10.19 & 11.67 & 14.17 \\
\hline Project & 3.98 & 5.35 & 3.31 & 7.21 & 7.10 & 4.56 & 4.94 \\
\hline Nonproject & 4.31 & 7.88 & 6.25 & 5.53 & 3.08 & 7.12 & 9.23 \\
\hline Budget support & 2.59 & 4.41 & 4.34 & 0.78 & 0.85 & 4.28 & 5.73 \\
\hline Loan to public enterprises & 1.72 & 3.48 & 1.92 & 4.74 & 2.23 & 2.84 & 3.50 \\
\hline Cash amortization & -0.82 & -0.90 & -0.75 & -1.19 & -1.42 & -1.61 & -0.73 \\
\hline Net domestic financing ${ }^{3}$ & 0.38 & -2.69 & -4.45 & 1.82 & 4.72 & 2.35 & -0.98 \\
\hline Adjusted for World Bank advanced budget support ${ }^{5}$ & 0.38 & -0.73 & -2.41 & -0.14 & 7.04 & 2.35 & -0.98 \\
\hline Privatization & 0.00 & 0.12 & 0.00 & 1.04 & 1.04 & 0.00 & 0.00 \\
\hline \multicolumn{8}{|l|}{ Memorandum items: } \\
\hline Project support & 15.17 & 24.58 & 17.11 & 29.87 & 27.24 & 33.42 & 44.25 \\
\hline Budget support & 10.67 & 13.33 & 13.17 & 10.33 & 11.56 & 15.82 & 18.02 \\
\hline Foreing financed net lending & 1.72 & 3.48 & 1.92 & 4.74 & 2.23 & 2.84 & 3.50 \\
\hline
\end{tabular}

Sources: Mozambican authorities; and IMF staff estimates and projections.

${ }^{1}$ Revenue minus noninterest current expenditure minus locally financed capital expenditure and locally financed net lending. Unallocated revenue and expenditure are included in the primary balance.

${ }^{2}$ Residual discrepancy between identified sources and use of funds.

${ }^{3}$ Excludes recapitilization bonds issued to the Bank of Mozambique. 
Table 2b. Mozambique: Government Finances, 2007-11 (cont.)

Percent of GDP

\begin{tabular}{|c|c|c|c|c|c|c|c|}
\hline & \multirow{2}{*}{$\begin{array}{r}2007 \\
\text { Act. }\end{array}$} & \multicolumn{2}{|c|}{2008} & \multicolumn{2}{|c|}{2009} & \multirow{2}{*}{$\begin{array}{r}2010 \\
\text { Proj. }\end{array}$} & \multirow{2}{*}{$\begin{array}{r}2011 \\
\text { Proj. }\end{array}$} \\
\hline & & CR No.9/49 & Act. & CR No.9/49 & Proj. & & \\
\hline Total revenue & 15.9 & 15.8 & 16.0 & 16.9 & 15.7 & 16.0 & 16.5 \\
\hline Tax revenue & 14.0 & 13.9 & 14.2 & 14.7 & 13.6 & 14.1 & 14.3 \\
\hline Taxes on income and profits & 4.5 & 4.7 & 4.9 & 4.5 & 3.7 & 5.1 & 5.0 \\
\hline Taxes on goods and services & 7.5 & 7.4 & 7.5 & 8.5 & 8.2 & 7.7 & 8.0 \\
\hline Of which: on petroleum products & 1.1 & 1.1 & 1.0 & 1.3 & 1.2 & 0.0 & 0.0 \\
\hline Taxes on international trade & 1.8 & 1.5 & 1.5 & 1.3 & 1.3 & 1.1 & 1.0 \\
\hline Other taxes & 0.2 & 0.3 & 0.3 & 0.3 & 0.3 & 0.3 & 0.3 \\
\hline Nontax revenue & 1.9 & 2.0 & 1.8 & 2.2 & 2.2 & 1.9 & 2.2 \\
\hline Total expenditure and net lending & 28.1 & 32.4 & 27.9 & 34.4 & 33.1 & 34.4 & 36.5 \\
\hline Current expenditure & 15.3 & 16.1 & 15.7 & 16.4 & 16.7 & 16.6 & 16.7 \\
\hline Compensation to employees & 7.7 & 8.0 & 8.1 & 8.4 & 8.9 & 8.4 & 8.2 \\
\hline Goods and services & 4.0 & 4.2 & 4.1 & 4.3 & 4.1 & 4.2 & 4.2 \\
\hline Interest on public debt & 0.6 & 0.6 & 0.5 & 0.5 & 0.5 & 0.6 & 0.8 \\
\hline Transfer payments & 2.9 & 3.2 & 3.1 & 3.1 & 3.1 & 3.5 & 3.5 \\
\hline \multirow[t]{2}{*}{ Domestic current primary balance } & 1.2 & 0.3 & 0.8 & 1.0 & -0.4 & 0.0 & 0.6 \\
\hline & & -3.1 & -3.1 & & & & \\
\hline Capital expenditure & 11.7 & 14.9 & 11.6 & 16.3 & 15.6 & 16.8 & 18.8 \\
\hline Grant finance projects & 3.2 & 5.0 & 2.5 & 5.2 & 3.1 & 4.1 & 4.8 \\
\hline Projet loans & 1.9 & 2.3 & 1.5 & 2.7 & 2.7 & 1.6 & 1.5 \\
\hline Locally financed & 3.8 & 4.0 & 4.2 & 5.0 & 5.2 & 5.2 & 5.0 \\
\hline Net lending & 1.1 & 1.4 & 0.6 & 1.8 & 0.8 & 0.9 & 1.0 \\
\hline Lending to public enterprises & 1.2 & 1.5 & 0.7 & 1.8 & 0.9 & 1.0 & 1.1 \\
\hline Locally financed & -0.1 & -0.1 & -0.1 & 0.0 & 0.0 & -0.1 & -0.1 \\
\hline Domestic primary balance, before grants, above the line ${ }^{1}$ & -2.5 & -3.6 & -3.4 & -4.0 & -5.6 & -5.2 & -4.3 \\
\hline Unallocated revenue $(+) /$ expenditure $(-)^{2}$ & -0.9 & 0.0 & 0.3 & 0.0 & 0.0 & 0.0 & 0.0 \\
\hline Overall balance, before grants (below the line) & -13.1 & -16.5 & -11.6 & -17.5 & -17.4 & -18.4 & -20.0 \\
\hline Grants received & 9.3 & 12.0 & 9.5 & 12.1 & 11.8 & 14.0 & 16.1 \\
\hline Project & 5.4 & 8.2 & 5.8 & 8.5 & 7.7 & 10.0 & 12.3 \\
\hline Nonproject & 3.9 & 3.8 & 3.7 & 3.6 & 4.1 & 4.0 & 3.8 \\
\hline Overall balance, after grants & -3.8 & -4.5 & -2.2 & -5.4 & -5.6 & -4.3 & -3.9 \\
\hline Net external financing & 3.6 & 5.6 & 4.0 & 4.3 & 3.4 & 3.5 & 4.2 \\
\hline Disbursements & 4.0 & 5.6 & 4.0 & 4.8 & 3.9 & 4.1 & 4.4 \\
\hline Project & 1.9 & 2.3 & 1.4 & 2.7 & 2.7 & 1.6 & 1.5 \\
\hline Nonproject & 2.1 & 3.4 & 2.6 & 2.1 & 1.2 & 2.5 & 2.9 \\
\hline Cash amortization & -0.4 & -0.4 & -0.3 & -0.4 & -0.5 & -0.6 & -0.2 \\
\hline Net domestic financing ${ }^{3}$ & 0.2 & -1.1 & -1.9 & 0.7 & 1.8 & 0.8 & -0.3 \\
\hline Privatization & 0.0 & 0.1 & 0.0 & 0.4 & 0.4 & 0.0 & 0.0 \\
\hline \multicolumn{8}{|l|}{ Memorandum items: } \\
\hline Project support & 7.3 & 10.5 & 7.2 & 11.2 & 10.4 & 11.6 & 13.8 \\
\hline Budget support & 5.1 & 5.7 & 5.5 & 3.9 & 4.4 & 5.5 & 5.6 \\
\hline Foreing financed net lending & 0.8 & 1.5 & 0.8 & 1.8 & 0.9 & 1.0 & 1.1 \\
\hline
\end{tabular}

Sources: Mozambican authorities; and IMF staff estimates and projections.

${ }^{1}$ Revenue minus noninterest current expenditure minus locally financed capital expenditure and locally financed net lending. Unallocated revenue and expenditure are included in the primary balance.

${ }^{2}$ Residual discrepancy between identified sources and use of funds.

${ }^{3}$ Excludes recapitilization bonds issued to the Bank of Mozambique. 
Table 2c. Mozambique: Government Finances, Quarterly, 2008, 2009 (cont.)

\begin{tabular}{|c|c|c|c|c|c|c|}
\hline & \multirow{2}{*}{\multicolumn{2}{|c|}{$\begin{array}{c}2008 \\
\text { Annual } \\
\end{array}$}} & \multicolumn{4}{|c|}{2009} \\
\hline & & & \multirow[t]{2}{*}{$\mathrm{H} 1$} & Q3 & \multirow[t]{2}{*}{ Q4 } & \multirow{2}{*}{$\begin{array}{c}\text { Annual } \\
\text { Prog. }\end{array}$} \\
\hline & Prog. & Act. & & Prog. & & \\
\hline Tax revenue & 32,566 & 34,018 & 17,408 & 9,488 & 8,490 & 35,385 \\
\hline Nontax revenue & 4,635 & 4,251 & 2,453 & 1,275 & 1,981 & 5,710 \\
\hline Compensation to employees & 18,859 & 19,273 & 10,809 & 6,358 & 6,153 & 23,320 \\
\hline Goods and services & 9,929 & 9,705 & 5,582 & 3,790 & 1,333 & 10,705 \\
\hline Interest on public debt & 1,306 & 1,257 & 713 & 197 & 482 & 1,392 \\
\hline Transfer payments & 7,623 & 7,396 & 4,286 & 2,110 & 1,783 & 8,178 \\
\hline Domestic current primary balance & 791 & 1,893 & -815 & $-1,495$ & 1,202 & $-1,108$ \\
\hline Locally financed & 9,514 & 10,091 & 4,826 & 3,183 & 5,438 & 13,447 \\
\hline Net lending & 3,311 & 1,478 & 836 & 499 & 845 & 2,180 \\
\hline Lending to public enterprises & 3,477 & 1,613 & 886 & 484 & 858 & 2,229 \\
\hline Locally financed & -166 & -135 & -51 & 15 & -13 & -49 \\
\hline Unallocated revenue $(+) /$ expenditure $(-)^{1}$ & 689 & 616 & 0 & 0 & 0 & 0 \\
\hline Grants received & 28,155 & 22,635 & 13,463 & 8,644 & 8,732 & 30,839 \\
\hline Project & 19,232 & 13,798 & 7,186 & 6,231 & 6,714 & 20,131 \\
\hline Nonproject & 8,923 & 8,837 & 6,277 & 2,412 & 2,018 & 10,708 \\
\hline Overall balance, after grants (incl. central bank recapitalization) & $-2,041$ & 8,656 & $-2,104$ & $-3,917$ & $-8,502$ & $-14,523$ \\
\hline \multicolumn{7}{|l|}{ Memorandum items: } \\
\hline Project support & 24,578 & 17,111 & 8,712 & 7,187 & 11,337 & 27,235 \\
\hline Budget support & 13,330 & 13,172 & 6,277 & 2,852 & 2,433 & 11,562 \\
\hline Foreing financed net lending & 3,477 & 1,916 & 886 & 484 & 858 & 2,229 \\
\hline
\end{tabular}

Sources: Mozambican authorities; and staff estimates and projections.

${ }^{1}$ Residual discrepancy between identified sources and use of funds.

${ }^{2}$ Excludes recapitilization bonds issued to the Bank of Mozambique. 
Table 3a. Mozambique: Monetary Survey, 2007-09 ${ }^{1}$

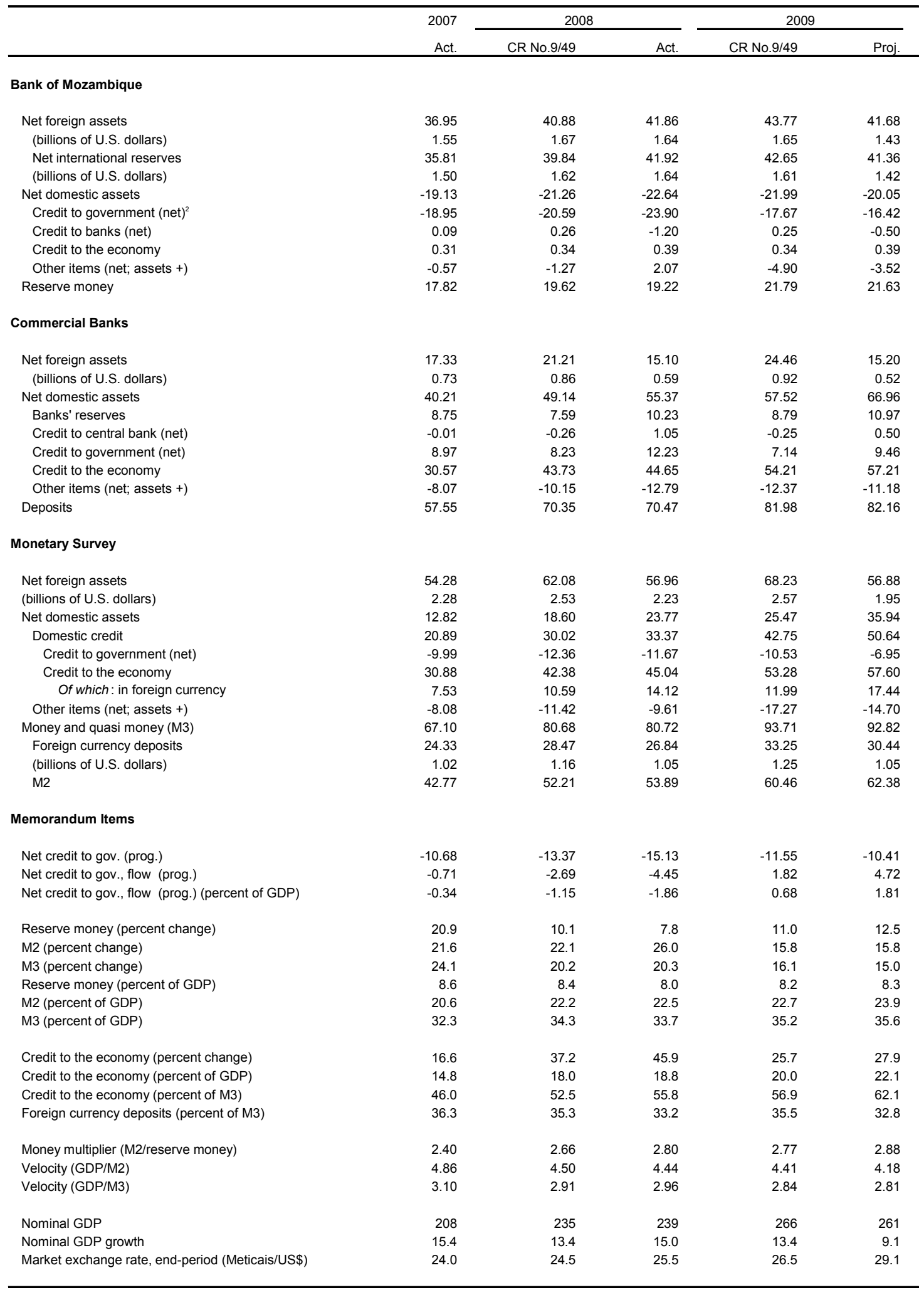

Sources: Bank of Mozambique; and IMF staff estimates and projections.

${ }^{1}$ Following the adoption of a Chart of Accounts in line with the IFRS, the monetary survey was revised back to January 1997 to incorporate more detailed information provided by banks regarding the residency and use of monetary instruments.

${ }^{2}$ Includes MDRI assistance which was transferred to a government blocked deposit account at the BM. The balance is being drawn down by 0.5 percent of per annum in line with an agreed increase in MDRI-financed government expenditures. 
Table 3b. Mozambique: Monetary Survey, Quarterly, 2008-09

\begin{tabular}{|c|c|c|c|c|c|c|c|c|}
\hline & \multicolumn{4}{|c|}{2008} & \multicolumn{4}{|c|}{2009} \\
\hline & $\begin{array}{r}\text { Q1 } \\
\text { Act. }\end{array}$ & $\begin{array}{r}\text { Q2 } \\
\text { Act. }\end{array}$ & $\begin{array}{r}\text { Q3 } \\
\text { Act. }\end{array}$ & $\begin{array}{r}\text { Q4 } \\
\text { Act. }\end{array}$ & $\begin{array}{r}\text { Q1 } \\
\text { Proj. }\end{array}$ & $\begin{array}{r}\text { Q2 } \\
\text { Proj. }\end{array}$ & $\begin{array}{r}\text { Q3 } \\
\text { Proj. }\end{array}$ & $\begin{array}{r}\text { Q4 } \\
\text { Proj. }\end{array}$ \\
\hline & \multicolumn{8}{|c|}{ (Billions of MT, unless otherwise stated) } \\
\hline \multicolumn{9}{|l|}{ Bank of Mozambique } \\
\hline $\begin{array}{l}\text { Net foreign assets } \\
\text { (billions of U.S. dollars) } \\
\text { Net international reserves } \\
\text { (billions of U.S. dollars) } \\
\text { Net domestic assets } \\
\text { Credit to government (net) } \\
\text { Credit to banks (net) } \\
\text { Credit to the economy } \\
\text { Other items (net; assets +) } \\
\text { of which: Valuation Adjustment } \\
\text { Reserve money } \\
\text { Currency in circulation } \\
\text { Bank deposits in BM }\end{array}$ & $\begin{array}{r}42.39 \\
1.74 \\
40.52 \\
1.67 \\
-26.59 \\
-25.08 \\
-0.18 \\
0.31 \\
-1.64 \\
-0.74 \\
15.80 \\
9.05 \\
6.75\end{array}$ & $\begin{array}{r}42.48 \\
1.77 \\
41.17 \\
1.71 \\
-25.45 \\
-25.04 \\
-0.19 \\
0.34 \\
-0.57 \\
-0.80 \\
17.03 \\
10.75 \\
6.28\end{array}$ & $\begin{array}{r}40.70 \\
1.68 \\
39.51 \\
1.64 \\
-23.20 \\
-24.81 \\
-0.48 \\
0.40 \\
1.70 \\
-0.89 \\
17.50 \\
10.76 \\
6.74\end{array}$ & $\begin{array}{r}41.86 \\
1.64 \\
41.92 \\
1.64 \\
-22.64 \\
-23.90 \\
-1.20 \\
0.39 \\
2.07 \\
-2.24 \\
19.22 \\
12.00 \\
7.22\end{array}$ & $\begin{array}{r}42.94 \\
1.55 \\
42.63 \\
1.54 \\
-25.04 \\
-25.88 \\
-0.29 \\
0.39 \\
0.74 \\
-2.40 \\
17.90 \\
10.59 \\
7.31\end{array}$ & $\begin{array}{r}43.64 \\
1.51 \\
43.32 \\
1.50 \\
-24.34 \\
-22.42 \\
-0.50 \\
0.39 \\
-1.80 \\
-4.22 \\
19.30 \\
11.68 \\
7.62\end{array}$ & $\begin{array}{r}43.36 \\
1.50 \\
43.04 \\
1.49 \\
-23.59 \\
-20.92 \\
-0.50 \\
0.39 \\
-2.56 \\
-4.25 \\
19.76 \\
11.86 \\
7.91\end{array}$ & $\begin{array}{r}41.68 \\
1.43 \\
41.36 \\
1.42 \\
-20.05 \\
-16.42 \\
-0.50 \\
0.39 \\
-3.52 \\
-2.48 \\
21.63 \\
12.76 \\
8.87\end{array}$ \\
\hline \multicolumn{9}{|l|}{ Monetary Survey } \\
\hline $\begin{array}{l}\text { Net foreign assets } \\
\text { Net domestic assets } \\
\text { Domestic credit } \\
\text { Credit to government (net) } \\
\text { Credit to the economy } \\
\text { Other items (net; assets +) } \\
\text { Money and quasi money (M3) } \\
\text { Foreign currency deposits } \\
\text { (millions of U.S. dollars) } \\
\text { M2 }\end{array}$ & $\begin{array}{r}60.46 \\
5.75 \\
16.53 \\
-15.33 \\
31.85 \\
-10.78 \\
66.21 \\
23.87 \\
0.98 \\
42.34\end{array}$ & $\begin{array}{r}60.37 \\
10.52 \\
20.96 \\
-13.80 \\
34.75 \\
-10.44 \\
70.89 \\
25.02 \\
1.04 \\
45.87\end{array}$ & $\begin{array}{r}57.39 \\
16.51 \\
26.00 \\
-12.53 \\
38.53 \\
-9.49 \\
73.90 \\
25.42 \\
1.05 \\
48.48\end{array}$ & $\begin{array}{r}56.96 \\
23.77 \\
33.37 \\
-11.67 \\
45.04 \\
-9.61 \\
80.72 \\
26.84 \\
1.05 \\
53.89\end{array}$ & $\begin{array}{r}56.91 \\
24.59 \\
35.85 \\
-13.02 \\
48.87 \\
-11.27 \\
81.50 \\
28.50 \\
1.03 \\
53.00\end{array}$ & $\begin{array}{r}57.37 \\
25.44 \\
39.34 \\
-12.15 \\
51.49 \\
-13.89 \\
82.81 \\
28.87 \\
1.00 \\
53.93\end{array}$ & $\begin{array}{r}57.39 \\
28.53 \\
42.69 \\
-9.91 \\
52.61 \\
-14.16 \\
85.92 \\
29.19 \\
1.01 \\
56.73\end{array}$ & $\begin{array}{r}56.88 \\
35.94 \\
50.64 \\
-6.95 \\
57.60 \\
-14.70 \\
92.82 \\
30.44 \\
1.05 \\
62.38\end{array}$ \\
\hline \multicolumn{9}{|l|}{ Memorandum Items } \\
\hline $\begin{array}{l}\text { Net credit to gov. (prog.) } \\
\text { Net credit to gov., flow (prog.) }\end{array}$ & $\begin{array}{r}-15.97 \\
-5.29\end{array}$ & $\begin{array}{r}-17.42 \\
-6.74\end{array}$ & $\begin{array}{r}-14.82 \\
-4.14\end{array}$ & $\begin{array}{r}-15.13 \\
-4.45\end{array}$ & $\begin{array}{r}-15.23 \\
-0.10\end{array}$ & $\begin{array}{r}-15.05 \\
0.08\end{array}$ & $\begin{array}{r}-12.98 \\
2.15\end{array}$ & $\begin{array}{r}-10.41 \\
4.72\end{array}$ \\
\hline $\begin{array}{l}\text { Reserve money (12-month percent change) } \\
\text { M2 (12-month percent change) } \\
\text { M3 (12-month percent change) } \\
\text { Credit to the economy (12-month percent change) }\end{array}$ & $\begin{array}{l}18.2 \\
24.2 \\
21.4 \\
22.0\end{array}$ & $\begin{array}{l}16.3 \\
29.0 \\
23.9 \\
27.5\end{array}$ & $\begin{array}{l}13.1 \\
25.3 \\
21.0 \\
34.1\end{array}$ & $\begin{array}{r}7.8 \\
26.0 \\
20.3 \\
45.9\end{array}$ & $\begin{array}{l}13.3 \\
25.2 \\
23.1 \\
53.4\end{array}$ & $\begin{array}{l}13.4 \\
17.6 \\
16.8 \\
48.2\end{array}$ & $\begin{array}{l}13.0 \\
17.0 \\
16.3 \\
36.5\end{array}$ & $\begin{array}{l}12.5 \\
15.8 \\
15.0 \\
27.9\end{array}$ \\
\hline $\begin{array}{l}\text { Money multiplier (M2/reserve money) } \\
\text { Money multiplier (M3/reserve money) } \\
\text { Velocity (GDP/M2) } \\
\text { Velocity (GDP/M3) }\end{array}$ & $\begin{array}{l}2.7 \\
4.2 \\
5.7 \\
3.6\end{array}$ & $\begin{array}{l}2.7 \\
4.2 \\
5.2 \\
3.4\end{array}$ & $\begin{array}{l}2.8 \\
4.2 \\
4.9 \\
3.2\end{array}$ & $\begin{array}{l}2.8 \\
4.2 \\
4.4 \\
3.0\end{array}$ & $\begin{array}{l}3.0 \\
4.6 \\
4.9 \\
3.2\end{array}$ & $\begin{array}{l}2.8 \\
4.3 \\
5.3 \\
3.5\end{array}$ & $\begin{array}{l}2.9 \\
4.3 \\
5.6 \\
3.7\end{array}$ & $\begin{array}{l}2.9 \\
4.3 \\
4.2 \\
2.8\end{array}$ \\
\hline
\end{tabular}

Sources: Bank of Mozambique; and IMF staff estimates and projections. 
Table 4. Mozambique: Balance of Payments, 2007-11

(Millions of U.S. dollars, unless otherwise specified)

\begin{tabular}{|c|c|c|c|c|c|c|c|}
\hline & \multirow{2}{*}{$\begin{array}{r}2007 \\
\text { Act. }\end{array}$} & \multicolumn{2}{|c|}{2008} & \multicolumn{2}{|c|}{2009} & \multirow{2}{*}{$\begin{array}{l}2010 \\
\text { Proj. }\end{array}$} & \multirow{2}{*}{$\begin{array}{r}2011 \\
\text { Proj. }\end{array}$} \\
\hline & & CR No.9/49 & Act. & CR No.9/49 & Proj. & & \\
\hline Trade balance & -680 & -1122 & -1150 & -1483 & -1796 & -1248 & -1321 \\
\hline Exports, f.o.b. & 2412 & 2511 & 2653 & 2190 & 1876 & 2358 & 2661 \\
\hline Megaprojects & 1843 & 1876 & 1851 & 1515 & 1192 & 1632 & 1883 \\
\hline Traditional exports & 569 & 635 & 802 & 674 & 684 & 727 & 778 \\
\hline Imports, c.i.f. & -3092 & -3633 & -3804 & -3673 & -3672 & -3606 & -3982 \\
\hline Megaprojects & -689 & -738 & -754 & -731 & -976 & -531 & -640 \\
\hline Other imports & -2403 & -2895 & -3050 & -2942 & -2696 & -3075 & -3342 \\
\hline Services and income balance & -896 & -936 & -871 & -624 & -339 & -981 & -1087 \\
\hline Receipts & 652 & 706 & 722 & 724 & 680 & 701 & 754 \\
\hline Expenditures $^{1}$ & -1548 & -1642 & -1593 & -1349 & -1019 & -1682 & -1841 \\
\hline Of which: Interest on public debt & -85 & -19 & -28 & -21 & -31 & -42 & -52 \\
\hline Unrequited official transfers & 790 & 1237 & 1046 & 1306 & 1116 & 1352 & 1665 \\
\hline Current account balance $^{2}$ & -785 & -821 & -975 & -802 & -1019 & -877 & -743 \\
\hline Capital and financial account balance & 862 & 805 & 1175 & 785 & 796 & 730 & 802 \\
\hline Trade credit (net) & -29 & 0 & -13 & 0 & 0 & 0 & 0 \\
\hline Foreign borrowing & 1440 & 914 & 640 & 847 & 552 & 472 & 540 \\
\hline Public (including IMF) & 325 & 539 & 384 & 508 & 333 & 247 & 272 \\
\hline Private & 1115 & 375 & 256 & 340 & 219 & 226 & 267 \\
\hline Amortization & -1026 & -427 & -396 & -476 & -476 & -485 & -592 \\
\hline Public & -32 & -37 & -37 & -47 & -47 & -19 & -25 \\
\hline Private $^{3}$ & -994 & -390 & -359 & -429 & -429 & -465 & -567 \\
\hline Foreign direct investment ${ }^{4}$ & 428 & 455 & 587 & 471 & 520 & 536 & 613 \\
\hline Short-term capital (net) & 49 & -137 & 357 & -57 & 200 & 206 & 242 \\
\hline Errors and omissions & 60 & 137 & -40 & 0 & 0 & 0 & 0 \\
\hline Overall balance & 136 & 121 & 159 & -17 & -223 & -146 & 60 \\
\hline Financing & -136 & -121 & -159 & 17 & 223 & 146 & -60 \\
\hline Of which: Change in gross reserve assets (increase -) & -136 & -121 & -159 & 17 & 223 & 146 & -60 \\
\hline \multicolumn{8}{|l|}{ Memorandum items: } \\
\hline \multicolumn{8}{|l|}{ Current account deficit (percent of GDP) } \\
\hline Before grants & 19.4 & 21.2 & 20.4 & 19.8 & 22.9 & 23.2 & 23.3 \\
\hline After grants & 9.7 & 8.4 & 9.9 & 7.5 & 10.9 & 9.1 & 7.2 \\
\hline Net international reserves ${ }^{5}$ & 1508 & 1625 & 1644 & 1608 & 1421 & 1275 & 1336 \\
\hline Gross international reserves & 1520 & 1641 & 1660 & 1625 & 1440 & 1294 & 1355 \\
\hline In months of imports of GNFS & 4.7 & 4.6 & 4.4 & 4.5 & 3.9 & 3.6 & 3.4 \\
\hline Gross aid (percent of GDP) & 13.2 & 17.5 & 13.3 & 17.6 & 15.7 & 18.1 & 20.5 \\
\hline Of which: Gross program aid (percent of GDP) & 5.1 & 5.7 & 5.6 & 4.7 & 4.4 & 5.5 & 5.6 \\
\hline
\end{tabular}

Sources: Mozambican authorities; and IMF staff estimates and projections.

${ }^{1}$ Includes income outflows related to megaprojects.

${ }^{2}$ Since this presentation follows the fourth balance of payments manual, MDRI and HIPC grants from the IMF are included in the current account.

${ }^{3}$ Includes HCB amortization in 2007.

${ }^{4}$ Projections are underestimated due to lack of information on new megaproject investments.

${ }^{5}$ Based on the new monetary survey from 2008. 
Table 4b. Mozambique: Balance of Payments, 2007-10

(Millions of U.S. dollars, unless otherwise specified)

\begin{tabular}{|c|c|c|c|c|c|c|c|}
\hline & \multirow[b]{2}{*}{2007} & \multirow[b]{2}{*}{2008} & \multirow[b]{2}{*}{2009} & \multirow[b]{2}{*}{2010} & \multicolumn{3}{|c|}{$\begin{array}{c}\text { Change Relative to Previous } \text { Year }^{1} \\
2009\end{array}$} \\
\hline & & & & & $\Delta$ in & $\Delta$ in & $\Delta$ in \\
\hline & Act. & Act. & Proj. & Proj. & value & price & volume \\
\hline Trade balance & -680 & -1150 & -1796 & -1248 & -646 & $\ldots$ & ... \\
\hline Exports, f.o.b. & 2412 & 2653 & 1876 & 2358 & -777 & $\ldots$ & ... \\
\hline Megaprojects & 1843 & 1851 & 1192 & 1632 & -659 & $\ldots$ & ... \\
\hline Of which: Aluminum & 1480 & 1452 & 676 & 1014 & -776 & -776 & 0 \\
\hline Traditional exports & 569 & 802 & 684 & 727 & -118 & $\ldots$ & ... \\
\hline Imports, c.i.f. & -3092 & -3804 & -3672 & -3606 & 131 & $\ldots$ & ... \\
\hline Megaprojects & -689 & -754 & -976 & -531 & -223 & $\ldots$ & ... \\
\hline Other imports & -2403 & -3050 & -2696 & -3075 & 354 & $\ldots$ & ... \\
\hline Of which: Oil & -266 & -467 & -261 & -330 & 206 & 226 & -20 \\
\hline Services and income balance & -896 & -871 & -339 & -981 & 532 & $\ldots$ & ... \\
\hline Receipts & 652 & 722 & 680 & 701 & -42 & $\ldots$ & ... \\
\hline Expenditures $^{2}$ & -1548 & -1593 & -1019 & -1682 & 575 & $\ldots$ & ... \\
\hline Current account balance, before grants & -1576 & -2021 & -2135 & -2229 & -113 & $\ldots$ & ... \\
\hline Unrequited official transfers & 790 & 1046 & 1116 & 1352 & 70 & $\ldots$ & .. \\
\hline Current account balance ${ }^{3}$ & -785 & -975 & -1019 & -877 & -44 & $\ldots$ & ... \\
\hline Capital and financial account balance & 862 & 1175 & 796 & 730 & -378 & $\ldots$ & ... \\
\hline Trade credit (net) & -29 & -13 & 0 & 0 & 13 & $\ldots$ & ... \\
\hline Foreign borrowing & 1440 & 640 & 552 & 472 & -88 & $\ldots$ & ... \\
\hline Public (including IMF) & 325 & 384 & 333 & 247 & -50 & $\ldots$ & ... \\
\hline Private $^{3}$ & 1115 & 256 & 219 & 226 & -37 & $\ldots$ & ... \\
\hline Amortization & -1026 & -396 & -476 & -485 & -80 & $\ldots$ & ... \\
\hline Public & -32 & -37 & -47 & -19 & -10 & $\ldots$ & ... \\
\hline Private $^{4}$ & -994 & -359 & -429 & -465 & -70 & $\ldots$ & ... \\
\hline Foreign direct investment ${ }^{5}$ & 428 & 587 & 520 & 536 & -67 & $\ldots$ & .. \\
\hline Short-term capital (net) & 49 & 357 & 200 & 206 & -157 & $\cdots$ & .. \\
\hline Errors and omissions & 60 & -40 & 0 & 0 & 40 & $\ldots$ & ... \\
\hline Overall balance & 136 & 159 & -223 & -146 & -382 & $\ldots$ & .. \\
\hline Financing & -136 & -159 & 223 & 146 & 382 & $\ldots$ & .. \\
\hline Financing gap & 0 & 0 & 0 & 0 & 0 & & \\
\hline \multicolumn{8}{|l|}{ Memorandum items: } \\
\hline \multicolumn{8}{|l|}{ Current account deficit (percent of GDP) } \\
\hline Before grants & 19.4 & 20.4 & 22.9 & 23.2 & 2.5 & $\ldots$ & ... \\
\hline After grants & 9.7 & 9.9 & 10.9 & 9.1 & 1.1 & $\ldots$ & ... \\
\hline Net international reserves ${ }^{6}$ & 1508 & 1644 & 1421 & 1275 & -223 & $\ldots$ & ... \\
\hline Gross international reserves & 1520 & 1660 & 1440 & 1294 & -221 & $\ldots$ & ... \\
\hline In months of imports of GNFS & 4.7 & 4.4 & 3.9 & 3.6 & -0.5 & $\ldots$ & ... \\
\hline Gross aid (percent of GDP) & 13.2 & 13.3 & 15.7 & 18.1 & 2.4 & $\ldots$ & ... \\
\hline Of which: Gross program aid (percent of GDP) & 5.1 & 5.6 & 4.4 & 5.5 & -1.1 & $\ldots$ & ... \\
\hline
\end{tabular}

Sources: Mozambican authorities; and IMF staff estimates and projections.

' The change in value, $P_{t} Q_{t}-P_{t-1} Q_{t-1}$, is decomposed into two parts: the change in price, $\left(P_{t}-P_{t-1}\right) Q_{t}$ and the change in volume, $P_{t-1}\left(Q_{t}-Q_{t-1}\right)$.

${ }^{2}$ Includes income outflows related to megaprojects.

${ }^{3}$ Since this presentation follows the fourth balance of payments manual, MDRI and HIPC grants from the IMF are included in the current account.

${ }^{4}$ Includes HCB amortization in 2007.

${ }^{5}$ Projections are underestimated due to lack of information on new megaproject investments.

${ }^{6}$ Based on the new monetary survey from 2008. 
Table 5. Mozambique: Indicators of Capacity to Repay the Fund ${ }^{1}$

\begin{tabular}{|c|c|c|c|c|c|c|c|c|c|c|c|c|c|c|}
\hline & \multicolumn{14}{|c|}{$\begin{array}{l}\text { Projection } \\
\end{array}$} \\
\hline & 2008 & 2009 & 2010 & 2011 & 2012 & 2013 & 2014 & 2015 & 2016 & 2017 & 2018 & 2019 & 2020 & Total \\
\hline \multicolumn{15}{|l|}{$\begin{array}{l}\text { Fund obligations based on existing credit } \\
\text { (millions of SDRs) }\end{array}$} \\
\hline Principal & - & - & 0.2 & 1.0 & 1.5 & 2.0 & 2.0 & 1.8 & 1.0 & 0.5 & - & - & - & 9.8 \\
\hline Charges and interest & 0.1 & 0.1 & 0.1 & 0.1 & 0.0 & 0.0 & 0.0 & 0.0 & - & - & - & - & - & 0.3 \\
\hline \multicolumn{15}{|c|}{$\begin{array}{l}\text { Fund obligations based on existing and prospective credit }{ }^{1} \\
\text { (millions of SDRs) }\end{array}$} \\
\hline Principal & - & - & 0.2 & 1.0 & 1.5 & 2.0 & 2.0 & 20.3 & 23.7 & 23.2 & 22.7 & 22.7 & 4.3 & 123.4 \\
\hline Charges and interest & - & 0.3 & 0.6 & 0.6 & 0.6 & 0.6 & 0.6 & 0.5 & 0.4 & 0.3 & 0.2 & 0.1 & - & 4.7 \\
\hline \multicolumn{15}{|l|}{ Total obligations based on existing and prospective credit ${ }^{1}$} \\
\hline In millions of SDRs & - & 0.3 & 0.7 & 1.6 & 2.1 & 2.6 & 2.5 & 20.8 & 24.1 & 23.5 & 22.9 & 22.8 & 4.3 & \\
\hline In millions of U.S. dollars & - & 0.4 & 1.1 & 2.3 & 3.0 & 3.8 & 3.8 & 30.7 & 35.6 & 34.7 & 33.9 & 33.7 & 6.3 & \\
\hline In percent of exports of goods and services & - & 0.0 & 0.0 & 0.1 & 0.1 & 0.1 & 0.1 & 0.7 & 0.8 & 0.7 & 0.6 & 0.6 & 0.1 & \\
\hline In percent of debt service ${ }^{2}$ & - & 0.6 & 1.9 & 3.0 & 3.1 & 3.5 & 2.9 & 19.8 & 20.5 & 18.9 & 17.1 & 15.3 & 2.8 & \\
\hline In percent of GDP & - & 0.0 & 0.0 & 0.0 & 0.0 & 0.0 & 0.0 & 0.2 & 0.2 & 0.2 & 0.2 & 0.2 & 0.0 & \\
\hline In percent of gross international reserves & - & 0.0 & 0.1 & 0.2 & 0.2 & 0.2 & 0.2 & 1.2 & 1.2 & 1.0 & 0.8 & 0.7 & 0.1 & \\
\hline In percent of quota & - & 0.2 & 0.7 & 1.4 & 1.8 & 2.2 & 2.2 & 18.3 & 21.2 & 20.7 & 20.1 & 20.0 & 3.8 & \\
\hline \multicolumn{15}{|l|}{ Outstanding Fund credit } \\
\hline In millions of SDRs & 9.7 & 94.9 & 123.2 & 122.2 & 120.8 & 118.8 & 116.9 & 96.6 & 72.9 & 49.7 & 27.0 & 4.3 & - & \\
\hline In millions of U.S. dollars & 15.4 & 140.9 & 181.0 & 179.4 & 177.6 & 175.3 & 172.8 & 142.9 & 107.8 & 73.5 & 39.9 & 6.3 & - & \\
\hline In percent of exports of goods and services & 0.5 & 5.7 & 6.1 & 5.4 & 5.0 & 4.6 & 4.4 & 3.3 & 2.3 & 1.5 & 0.7 & 0.1 & - & \\
\hline In percent of debt service ${ }^{2}$ & 30.7 & 223.4 & 309.9 & 231.6 & 181.8 & 163.1 & 134.2 & 92.0 & 62.0 & 40.1 & 20.1 & 2.9 & - & \\
\hline In percent of GDP & 0.2 & 1.5 & 1.9 & 1.7 & 1.6 & 1.4 & 1.3 & 1.0 & 0.7 & 0.5 & 0.2 & 0.0 & - & \\
\hline In percent of gross international reserves & 0.9 & 9.8 & 14.0 & 13.2 & 10.2 & 8.9 & 8.5 & 5.7 & 3.5 & 2.0 & 0.9 & 0.1 & - & \\
\hline In percent of quota & 8.7 & 83.6 & 108.4 & 107.6 & 106.3 & 104.6 & 102.9 & 85.0 & 64.2 & 43.8 & 23.8 & 3.8 & - & \\
\hline Net use of Fund credit (millions of SDRs) & 84.5 & 85.2 & 28.2 & $(1.0)$ & (1.5) & (2.0) & (2.0) & $(20.3)$ & (23.7) & (23.2) & (22.7) & (22.7) & (4.3) & (9.8) \\
\hline Disbursements $^{1}$ & 84.5 & 85.2 & 28.4 & - & - & - & - & - & - & - & - & - & - & 113.6 \\
\hline Repayments & - & - & 0.2 & 1.0 & 1.5 & 2.0 & 2.0 & 20.3 & 23.7 & 23.2 & 22.7 & 22.7 & 4.3 & 123.4 \\
\hline \multicolumn{15}{|l|}{ Memorandum items: } \\
\hline Exports of goods and services (millions of U.S. dollars) & 3,297 & 2,483 & 2,984 & 3,334 & 3,573 & 3,774 & 3,965 & 4,275 & 4,603 & 4,956 & 5,335 & 5,744 & 6,183 & \\
\hline Debt service (millions of U.S. dollars) ${ }^{2}$ & 50 & 63 & 58 & 77 & 98 & 107 & 129 & 155 & 174 & 183 & 198 & 221 & 225 & \\
\hline Nominal GDP (millions of U.S. dollars) & 9,897 & 9,316 & 9,608 & 10,332 & 11,158 & 12,106 & 12,859 & 13,798 & 14,690 & 15,632 & 16,630 & 17,688 & 18,809 & \\
\hline Gross international reserves (millions of U.S. dollars) & 1,660 & 1,440 & 1,294 & 1,355 & 1,737 & 1,960 & 2,037 & 2,515 & 3,078 & 3,656 & 4,327 & 5,043 & 5,772 & \\
\hline Quota (millions of SDRs) & 112.6 & 113.6 & 113.6 & 113.6 & 113.6 & 113.6 & 113.6 & 113.6 & 113.6 & 113.6 & 113.6 & 113.6 & 113.6 & \\
\hline SDR exchange rate (SDR/USD) & 0.63 & 0.67 & 0.68 & 0.68 & 0.68 & 0.68 & 0.68 & 0.68 & 0.68 & 0.68 & 0.68 & 0.68 & 0.68 & \\
\hline
\end{tabular}

Sources: Mozambican authorities; and IMF staff estimates and projections.

${ }^{1}$ Assumes an ESF-HAC arrangement of SDR 113.6 million (100 percent of quota) to be disbursed in three tranches: SDR 85.2 million (75 percent of quota) upon completion of the fourth PSI review;

SDR 14.2 million (12.5 percent of quota) upon completion of the fifth PSI review; and SDR 14.2 million (12.5 percent of quota) upon completion of the sixth PSI review.

${ }^{2}$ Total debt service includes IMF repayments. 
Table 6. Mozambique: Schedule of Disbursements under the 12-Month ESF Arrangement (millions of SDR)

\begin{tabular}{clc}
\hline Amount & Conditions for disbursement & Date available \\
85.2 & On approval of arrangement & July 10, 2009 \\
14.2 & Completeion of the first ESF review & Dec 7, 2009 \\
14.2 & Completeion of the second ESF review & June 7, 2010 \\
& & \\
\hline 113.6 & & \\
\hline
\end{tabular}

Sources: IMF staff projections. 
Table 7. Mozambique: Indicators of External Vulnerability, 2002-08

(percent of GDP, unless otherwise indicated)

\begin{tabular}{|c|c|c|c|c|c|c|c|}
\hline & $\begin{array}{r}2002 \\
\text { Act. }\end{array}$ & $\begin{array}{r}2003 \\
\text { Act. }\end{array}$ & $\begin{array}{r}2004 \\
\text { Act. }\end{array}$ & $\begin{array}{r}2005 \\
\text { Act. }\end{array}$ & $\begin{array}{r}2006 \\
\text { Act. }\end{array}$ & $\begin{array}{r}2007 \\
\text { Act. }\end{array}$ & $\begin{array}{r}2008 \\
\text { Proj. }\end{array}$ \\
\hline \multicolumn{8}{|l|}{ Financial indicators } \\
\hline Net present value of public sector debt $1 / 2 /$ & 21.3 & 25.8 & 26.9 & 26.3 & 9.8 & 16.8 & 17.6 \\
\hline Broad money (percent change, 12-month basis) & 21.5 & 18.7 & 5.9 & 27.1 & 23.4 & 25.2 & 20.3 \\
\hline Private sector credit (percent change, 12-month basis) & 4.2 & -1.4 & -5.6 & 57.0 & 29.2 & 12.6 & 45.9 \\
\hline \multicolumn{8}{|l|}{31 day $T$-bill yield $3 /$} \\
\hline \multicolumn{8}{|l|}{31 day $T$-bill yield (real) $4 /$} \\
\hline \multicolumn{8}{|l|}{ External indicators } \\
\hline Exports of goods (percent change, 12 -month basis in U.S. dollars) & 15.2 & 28.9 & 44.1 & 16.0 & 36.5 & 1.3 & 10.0 \\
\hline Imports of goods c.i.f. (percent change, 12-month basis in U.S. dollars) & 45.1 & 12.8 & 16.9 & 21.2 & 19.7 & 4.8 & 23.0 \\
\hline Terms of trade (percent change, 12-month basis) & -3.7 & -1.3 & 9.8 & 5.4 & 27.4 & 4.2 & -15.5 \\
\hline Current account balance (after grants) & -9.7 & -9.4 & -5.4 & -9.8 & 18.5 & -9.7 & -9.9 \\
\hline Capital and financial account balance & 20.7 & 9.3 & 5.9 & 5.3 & -22.2 & 10.6 & 11.9 \\
\hline Of which: foreign direct investment (net) & 8.3 & 7.2 & 4.3 & 1.6 & 2.1 & 5.3 & 5.9 \\
\hline Gross international reserves (in millions of U.S. dollars) & 825 & 988 & 1,160 & 1,103 & 1,241 & 1,520 & 1,660 \\
\hline (In months of imports of goods and services) & 5.3 & 5.3 & 5.5 & 4.6 & 4.4 & 4.7 & 4.4 \\
\hline (In months of imports of goods and services, excluding megaprojects) & 7.4 & 6.2 & 6.3 & 5.7 & 6.7 & 6.0 & 5.5 \\
\hline Net international reserves (in millions of U.S. dollars) & 625 & 779 & 961 & 943 & 1,231 & 1,504 & 1,644 \\
\hline Net international reserves (in percent of foreign currency deposits) & 129 & 145 & 158 & 144 & 170 & 147 & 156 \\
\hline Net foreign assets of commercial banks (in millions of U.S. dollars) & 342 & 306 & 264 & 342 & 524 & 728 & 592 \\
\hline Total short-term external debt to reserves (in percent) & 8.4 & 7.6 & 4.9 & 7.3 & 3.1 & 2.6 & 2.6 \\
\hline Net present value of total external debt $1 / 2 /$ & 21.3 & 25.8 & 26.9 & 26.3 & 9.8 & 16.8 & 17.6 \\
\hline Of which: net present value of public sector external debt $2 /$ & 22 & 25 & 8 & 18 & 18 & 19 & 19 \\
\hline Net present value of public external debt-to-exports of goods and services (in percent) $2 /$ & 91 & 102 & 99 & 98 & 63 & 54 & 52 \\
\hline Public external debt-service payments to exports of goods and services (in percent) $3 /$ & 5.2 & 5.1 & 3.2 & 3.0 & 0.8 & 3.4 & 1.5 \\
\hline Exchange rate (MT per U.S. dollar; end of period) $4 /$ & 23.9 & 23.9 & 18.9 & 24.2 & 26.0 & 23.8 & 25.5 \\
\hline REER depreciation (-) (12 month basis) & -14.3 & -8.5 & 17.5 & -6.3 & 1.8 & 0.0 & 0.0 \\
\hline
\end{tabular}

Sources: Mozambican authorities; and IMF staff estimates and projections.

1/ NPV of total public external debt only

2/ Historical NPV estimates are based on previous debt sustainability analyses.

3/ Cash interest and amortization payments, after HIPC assistance.

4/ Official exchange rate. 
Table 8. Mozambique: Financial Soundness Indicators for Banking Sector, 2001-08 (Percent unless otherwise indicated)

\begin{tabular}{|c|c|c|c|c|c|c|c|c|}
\hline & 2001 & 2002 & 2003 & 2004 & 2005 & 2006 & 2007 & 2008 \\
\hline \multicolumn{9}{|l|}{ Capital adequacy } \\
\hline Regulatory capital to risk-weighted assets & 5.49 & 14.01 & 17.03 & 18.65 & 16.00 & 12.53 & 14.21 & 13.9 \\
\hline Regulatory Tier I capital to risk-weighted assets & 6.03 & 11.97 & 14.67 & 15.97 & 13.57 & 10.72 & 12.32 & 12.41 \\
\hline Capital (net worth) to assets & 8.22 & 9.42 & 9.01 & 9.51 & 8.04 & 6.06 & 6.35 & 6.65 \\
\hline \multicolumn{9}{|l|}{ Asset composition and quality } \\
\hline \multicolumn{9}{|l|}{ Sectoral distribution of loans to total loans } \\
\hline Agriculture & 18.00 & 15.00 & 12.73 & 9.45 & 8.49 & 6.39 & 9.42 & 8.05 \\
\hline Industry & 25.00 & 22.00 & 16.94 & 11.94 & 16.77 & 22.15 & 19.51 & 16.72 \\
\hline Construction & 4.00 & 4.00 & 5.20 & 3.35 & 4.13 & 5.64 & 5.74 & 4.24 \\
\hline Commerce & 20.00 & 17.00 & 18.13 & 21.29 & 27.36 & 27.40 & 24.44 & 25.62 \\
\hline Transportation and communication & 7.00 & 5.00 & 7.08 & 7.19 & 5.83 & 6.15 & 12.18 & 11.15 \\
\hline Other & 27.00 & 36.00 & 37.14 & 36.18 & 35.05 & 32.27 & 28.7 & 34.22 \\
\hline of which: Private $1 /$ & & & 7.63 & 12.12 & 13.03 & 13.54 & 14.38 & 19.94 \\
\hline Housing & & & 7.10 & 8.18 & 4.24 & 4.07 & 3.69 & 3.67 \\
\hline Diverse $2 /$ & & & 22.41 & 15.87 & 17.78 & 14.66 & 10.63 & 10.63 \\
\hline Foreign exchange loans to total loans & 64.69 & 69.87 & 70.77 & 67.33 & 63.91 & 45.04 & 31.46 & 40.1 \\
\hline Nonperforming loans to gross loans $3 /$ & 23.43 & 21.98 & 14.43 & 6.42 & 3.82 & 3.26 & 0.90 & 1.95 \\
\hline Nonperforming loans net of provisions to capital $3 /$ & 11.00 & 9.39 & 7.91 & 1.70 & 0.89 & 2.17 & 15.46 & 12.16 \\
\hline \multicolumn{9}{|l|}{ Earnings and profitability } \\
\hline Return on assets & 0.14 & 1.59 & 1.24 & 1.43 & 1.81 & 3.51 & 1.92 & 3.5 \\
\hline Return on equity & 3.51 & 22.10 & 16.29 & 18.73 & 27.42 & 55.39 & 25.13 & 40.7 \\
\hline Interest margin to gross income & 10.22 & 61.39 & 56.83 & 60.15 & 58.01 & 63.07 & 56.15 & 57.08 \\
\hline Noninterest expenses to gross income & 16.92 & 67.03 & 72.18 & 71.24 & 65.85 & 54.10 & 58.34 & 61.11 \\
\hline Personnel expenses to noninterest expenses & 51.71 & 44.67 & 45.68 & 45.21 & 45.35 & 44.35 & 44.43 & $\ldots$ \\
\hline Trading and fee income to gross income & 33.09 & 39.68 & 45.49 & 41.87 & 43.78 & 32.15 & 43.37 & 43.3 \\
\hline Spread between reference loan and deposit rates (90 days, local currency) & 14.00 & 19.00 & 17.39 & 14.67 & 11.48 & 14.57 & 11.17 & 10.7 \\
\hline \multicolumn{9}{|l|}{ Funding and liquidity } \\
\hline Liquid assets to total assets 4/ & 34.64 & 53.93 & 57.74 & 58.12 & 55.52 & 51.87 & 53.25 & 49.27 \\
\hline Customer deposits to total (non-interbank) loans & 217.00 & 240.00 & 227.97 & 283.07 & 191.90 & 179.34 & 198.21 & 170.62 \\
\hline Foreign exchange liabilities to total liabilities & 63.30 & 61.26 & 46.39 & 41.38 & 45.26 & 42.75 & 35.52 & 32.18 \\
\hline
\end{tabular}

$1 /$ Includes credit cards and consumer credit lines for vehicle and durable goods.

2/ Includes credit to all other sectors not discriminated above or yet to be identified.

3/ Nonperforming loans are defined according to Mozambican accounting standards (they include only part of the past-due loans).

4/ Includes deposits at parent banks. 
Table 9. Mozambique: Expenditure in PARPA Priority Sectors, 2007-2009 1/

(Millions of meticais, unless otherwise indicated)

\begin{tabular}{|c|c|c|c|c|c|c|c|c|}
\hline & 2002 & 2003 & 2004 & 2005 & 2006 & 2007 & 2008 & 2009 \\
\hline Total revenues & 12,057 & 14,579 & 16,838 & 21,418 & 26,997 & 33,058 & 38,268 & 41,095 \\
\hline Total expenditure, excluding bank restructuring costs and net lending & 25,643 & 27,345 & 32,728 & 34,063 & 46,810 & 56,152 & 65,375 & 84,277 \\
\hline Total expenditure (excl.bank restruct. costs, net lending, and interest payments) & 24,369 & 26,027 & 31,407 & 32,815 & 45,430 & 54,875 & 64,118 & 82,885 \\
\hline Interest payments on public debt & 1,274 & 1,318 & 1,321 & 1,248 & 1,380 & 1,277 & 1,257 & 1,392 \\
\hline Total expenditure in PARPA priority sectors & 15,323 & 16,631 & 19,775 & 21,756 & 29,575 & 34,187 & 47,352 & 58,767 \\
\hline in percent of GDP & 15.4 & 15.0 & 15.4 & 14.3 & 17.0 & 16.4 & 19.8 & 22.5 \\
\hline in percent of total expenditure (excl. bank restruct costs, net lending, and intere & 62.9 & 63.9 & 63.0 & 66.3 & 65.1 & 62.3 & 73.9 & 70.9 \\
\hline Education & 4,217 & 5,257 & 6,563 & 6,542 & 9,109 & 11,949 & 10,847 & 12,989 \\
\hline Primary & 3,608 & 4,425 & 5,532 & 5,572 & 7,556 & 10,682 & 0 & 14,935 \\
\hline Postsecondary & 610 & 833 & 1,031 & 970 & 1,602 & 1,463 & 0 & 3,473 \\
\hline Health & 2,953 & 2,759 & 3,308 & 4,159 & 6,628 & 6,912 & 7,000 & 9,854 \\
\hline HIVIAIDS & 188 & 78 & 128 & 218 & 665 & 390 & 0 & 695 \\
\hline Infrastructure development & 3,861 & 3,592 & 4,137 & 6,131 & 7,435 & 7,827 & 12,475 & 11,673 \\
\hline Roads & 1,860 & 2,863 & 3,234 & 4,641 & 4,501 & 5,142 & 0 & 5,557 \\
\hline Sanitation and public works & 2,001 & 729 & 904 & 1,490 & 2,742 & 1,762 & 0 & 6,426 \\
\hline Agriculture and rural development & 1,243 & 1,275 & 1,373 & 1,290 & 1,522 & 2,067 & 2,302 & 4,803 \\
\hline Governance and judicial system & 1,900 & 2,447 & 3,050 & 2,913 & 5,671 & 4,433 & 4,746 & 5,111 \\
\hline Security and public order & 1,267 & 495 & 1,821 & 1,453 & 1,943 & 1,799 & 0 & 4,255 \\
\hline Governance & 235 & 1,458 & 502 & 601 & 2,719 & 1,056 & 0 & 2,258 \\
\hline Judicial system & 399 & 495 & 727 & 859 & 1,009 & 1,042 & 0 & 2,084 \\
\hline Other priority areas & 962 & 1,041 & 1,215 & 727 & 510 & 507 & 677 & 626 \\
\hline Social actions & 211 & 182 & 208 & 210 & 329 & 299 & 513 & 663 \\
\hline Labor and employment & 117 & 78 & 128 & 108 & 181 & 138 & 256 & 332 \\
\hline Mineral resources and energy & 633 & 781 & 879 & 409 & 0 & 634 & 769 & 995 \\
\hline \multicolumn{9}{|l|}{ Total PARPA expenditures in percent of GDP } \\
\hline Education & 4.2 & 4.7 & 5.1 & 4.3 & 5.2 & 5.7 & 4.5 & 5.0 \\
\hline Primary & 3.6 & 4.0 & 4.3 & 3.7 & 4.4 & 5.1 & 0.0 & 5.7 \\
\hline Postsecondary & 0.6 & 0.8 & 0.8 & 0.6 & 0.9 & 0.7 & 0.0 & 1.3 \\
\hline Health & 3.0 & 2.5 & 2.6 & 2.7 & 3.8 & 3.3 & 2.9 & 3.8 \\
\hline HIVIAIDS & 0.2 & 0.1 & 0.1 & 0.1 & 0.4 & 0.2 & 0.0 & 0.3 \\
\hline Infrastructure development & 3.9 & 3.2 & 3.2 & 4.0 & 4.3 & 3.8 & 5.2 & 4.5 \\
\hline Roads & 1.9 & 2.6 & 2.5 & 3.1 & 2.6 & 2.5 & 0.0 & 2.1 \\
\hline Sanitation and public works & 2.0 & 0.7 & 0.7 & 1.0 & 1.6 & 0.8 & 0.0 & 2.5 \\
\hline Agriculture and rural development & 1.2 & 1.1 & 1.1 & 0.9 & 0.9 & 1.0 & 1.0 & 1.8 \\
\hline Governance and judicial system & 1.9 & 2.2 & 2.4 & 1.9 & 3.3 & 2.1 & 2.0 & 2.0 \\
\hline Security and public order & 1.3 & 0.4 & 1.4 & 1.0 & 1.1 & 0.9 & 0.0 & 1.6 \\
\hline Governance & 0.2 & 1.3 & 0.4 & 0.4 & 1.6 & 0.5 & 0.0 & 0.9 \\
\hline Judicial system & 0.4 & 0.4 & 0.6 & 0.6 & 0.6 & 0.5 & 0.0 & 0.8 \\
\hline Other priority areas & 1.0 & 0.9 & 0.9 & 0.5 & 0.3 & 0.2 & 0.3 & 0.2 \\
\hline Social actions & 0.2 & 0.2 & 0.2 & 0.1 & 0.2 & 0.1 & 0.2 & 0.3 \\
\hline Labor and employment & 0.1 & 0.1 & 0.1 & 0.1 & 0.1 & 0.1 & 0.1 & 0.1 \\
\hline Mineral resources and energy & 0.6 & 0.7 & 0.7 & 0.3 & 0.0 & 0.3 & 0.3 & 0.4 \\
\hline \multicolumn{9}{|l|}{ Total PARPA expenditures in percent of total expenditures } \\
\hline Education & 17.3 & 20.2 & 20.9 & 19.9 & 20.1 & 21.8 & 16.9 & 15.7 \\
\hline Primary & 14.8 & 17.0 & 17.6 & 17.0 & 16.6 & 19.5 & 0.0 & 18.0 \\
\hline Postsecondary & 2.5 & 3.2 & 3.3 & 3.0 & 3.5 & 2.7 & 0.0 & 4.2 \\
\hline Health & 12.1 & 10.6 & 10.5 & 12.7 & 14.6 & 12.6 & 10.9 & 11.9 \\
\hline HIVIAIDS & 0.8 & 0.3 & 0.4 & 0.7 & 1.5 & 0.7 & 0.0 & 0.8 \\
\hline Infrastructure development & 15.8 & 13.8 & 13.2 & 18.7 & 16.4 & 14.3 & 19.5 & 14.1 \\
\hline Roads & 7.6 & 11.0 & 10.3 & 14.1 & 9.9 & 9.4 & 0.0 & 6.7 \\
\hline Sanitation and public works & 8.2 & 2.8 & 2.9 & 4.5 & 6.0 & 3.2 & 0.0 & 7.8 \\
\hline Agriculture and rural development & 5.1 & 4.9 & 4.4 & 3.9 & 3.4 & 3.8 & 3.6 & 5.8 \\
\hline Governance and judicial system & 7.8 & 9.4 & 9.7 & 8.9 & 12.5 & 8.1 & 7.4 & 6.2 \\
\hline Security and public order & 5.2 & 1.9 & 5.8 & 4.4 & 4.3 & 3.3 & 0.0 & 5.1 \\
\hline Governance & 1.0 & 5.6 & 1.6 & 1.8 & 6.0 & 1.9 & 0.0 & 2.7 \\
\hline Judicial system & 1.6 & 1.9 & 2.3 & 2.6 & 2.2 & 1.9 & 0.0 & 2.5 \\
\hline Other priority areas & 3.9 & 4.0 & 3.9 & 2.2 & 1.1 & 0.9 & 1.1 & 0.8 \\
\hline Social actions & 0.9 & 0.7 & 0.7 & & & 0.5 & 0.8 & 0.8 \\
\hline Labor and employment & 0.5 & 0.3 & 0.4 & & & 0.3 & 0.4 & 0.4 \\
\hline Mineral resources and energy & 2.6 & 3.0 & 2.8 & & & 1.2 & 1.2 & 1.2 \\
\hline \multicolumn{9}{|l|}{ Memorandum items: } \\
\hline Exchange rate (average) & 23,666 & 23,782 & 22,580 & 23,061 & 24,982 & 25,671 & 24,174 & 28,017 \\
\hline Nominal GDP & 99,479 & 110,973 & 128,668 & 151,707 & 180,242 & 207,986 & 239,249 & 261,015 \\
\hline \multicolumn{9}{|l|}{ HIPC debt relief accruing to the budget } \\
\hline In millions of U.S. dollars & 23 & 10 & 21 & 4 & 7 & 10 & 5 & 5 \\
\hline In billions of meticais & 44 & 237 & 484 & 72 & 135 & 242 & 146 & 150 \\
\hline In percent of GDP & 0 & 0 & 0 & 0 & 0 & 0 & 0 & 0 \\
\hline PARPA Priority Spending in million US dollars & 647 & 699 & 876 & 943 & 1,184 & 1,332 & 1,959 & 2,098 \\
\hline
\end{tabular}

Source: Mozambican authorities (Ministry of Finance); and staff estimates and projections.

1/ PARPA stands for National Action Plan for the Reduction of Absolute Poverty, which is the Portuguese acronym. 
Table 10. Mozambique: Millennium Development Goals, 1990-2007

\begin{tabular}{|c|c|c|c|c|}
\hline & 1990 & 1995 & 2000 & 2007 \\
\hline \multicolumn{5}{|l|}{ Goal 1: Eradicate extreme poverty and hunger } \\
\hline Employment to population ratio, $15+$, total (\%) & 63 & 62 & 62 & 61 \\
\hline Employment to population ratio, ages $15-24$, total $(\%)$ & 53 & 51 & 47 & 45 \\
\hline GDP per person employed (annual \% growth) & 0 & 2 & 3 & 3 \\
\hline Malnutrition prevalence, weight for age ( $\%$ of children under 5 ) & .. & .. & .. & 23.2 \\
\hline Prevalence of undernourishment (\% of population) & 17 & 15 & .. & 14 \\
\hline \multicolumn{5}{|l|}{ Goal 2: Achieve universal primary education } \\
\hline Literacy rate, youth female (\% of females ages $15-24$ ) & 79 & .. & 84 & 87 \\
\hline Literacy rate, youth male (\% of males ages $15-24$ ) & 88 & .. & 90 & 91 \\
\hline Primary completion rate, total (\% of relevant age group) & 79 & .. & 83 & 86 \\
\hline Total enrollment, primary (\% net) & .. & .. & 85 & 89 \\
\hline \multicolumn{5}{|l|}{ Goal 3: Promote gender equality and empower women } \\
\hline Proportion of seats held by women in national parliaments (\%) & 13 & 12 & 14 & 18 \\
\hline Ratio of female to male enrollments in tertiary education & .. & .. & 96 & 106 \\
\hline Ratio of female to male primary enrollment & 89 & .. & 92 & 95 \\
\hline Ratio of female to male secondary enrollment & 83 & .. & 93 & 95 \\
\hline Share of women employed in the nonagricultural sector (\% of total nonagricultural employment) & 34.6 & 37 & 36.3 & .. \\
\hline \multicolumn{5}{|l|}{ Goal 4: Reduce child mortality } \\
\hline Immunization, measles ( $\%$ of children ages $12-23$ months) & 73 & 74 & 73 & 82 \\
\hline Mortality rate, infant (per 1,000 live births) & 63 & 60 & 55 & 47 \\
\hline Mortality rate, under-5 (per 1,000) & 93 & 88 & 83 & 68 \\
\hline \multicolumn{5}{|l|}{ Goal 5: Improve maternal health } \\
\hline Adolescent fertility rate (births per 1,000 women ages $15-19$ ) & .. & 65 & 60 & 52 \\
\hline Births attended by skilled health staff ( $\%$ of total) & 50 & .. & .. & 65 \\
\hline Maternal mortality ratio (modeled estimate, per 100,000 live births) & 430 & .. & .. & 400 \\
\hline \multicolumn{5}{|l|}{ Goal 6: Combat HIVIAIDS, malaria, and other diseases } \\
\hline Incidence of tuberculosis (per 100,000 people) & 125 & 128 & 136 & 139 \\
\hline Prevalence of HIV, female (\% ages $15-24)$ & .. & .. & .. & 0.7 \\
\hline Prevalence of HIV, male (\% ages $15-24)$ & .. & .. & .. & 0 \\
\hline Prevalence of HIV, total (\% of population ages $15-49$ ) & 0.3 & 0.7 & 0.9 & 0.8 \\
\hline Tuberculosis cases detected under DOTS (\%) & .. & 14 & 30 & 63 \\
\hline \multicolumn{5}{|l|}{ Goal 7: Ensure environmental sustainability } \\
\hline CO2 emissions (metric tons per capita) & 4.3 & 4.1 & 4.1 & 4.5 \\
\hline Forest area (\% of land area) & 31 & 31 & 31 & 30 \\
\hline Improved sanitation facilities (\% of population with access) & 51 & 54 & 57 & 60 \\
\hline Improved water source (\% of population with access) & 76 & 79 & 82 & 86 \\
\hline \multicolumn{5}{|l|}{ Goal 8: Develop a global partnership for development } \\
\hline Aid per capita (current US\$) & 11 & 12 & 10 & 16 \\
\hline Internet users (per 100 people) & 0.1 & 0.8 & 6.7 & 21.8 \\
\hline Mobile cellular subscriptions (per 100 people) & 0 & 2 & 12 & 51 \\
\hline Telephone lines (per 100 people) & 10 & 12 & 16 & 20 \\
\hline
\end{tabular}

Source: World Development Indicators database 


\section{APPENDIX I. \\ REPUBLIC OF MOZAMBIQUE: LETTER OF INTENT}

May 13, 2009

Mr. Dominique Strauss-Kahn

Managing Director

International Monetary Fund

Washington, D.C. 20431

U.S.A.

Dear Mr. Strauss-Kahn:

1. The Government of Mozambique is implementing an economic and financial program consistent with its medium-term Plano de Acção para a Redução da Pobreza Absoluta II (PARPA II) and supported by the Fund under the Policy Support Instrument (PSI). Program implementation has progressed well, contributing to high and sustainable economic growth. The Government seeks to consolidate these achievements and explore initiatives to further promote growth and reduce poverty.

2. Implementation of the PSI-supported program in the second half of 2008 has been satisfactory. All quantitative assessment criteria (ACs) to end-December 2008 were met. Implementation of the two structural benchmarks for end-March 2009- the approval of a PFM vision paper and the issuance of a decree streamlining the business inspection process - was delayed and both are now expected to be implemented by end-July 2009. On this basis, the Government requests completion of the fourth review under the PSI-supported program.

3. Macroeconomic performance during 2008 was good despite pressures from high fuel and food prices in mid-2008 and the onset of the global economic crisis in late 2008. As in its response to the surge in international prices, the Government intends to respond to the current global economic crisis in a timely and appropriate manner, in consultation with Fund staff.

4. To mitigate the adverse impact of the global economic crisis, the Government will ease fiscal and monetary policies during 2009. However, the scope for supportive policies is constrained by a large projected drop in external reserves resulting directly from the sudden deterioration in the external environment. Indeed, export receipts had started to decline by late 2008 and both public and private capital inflows are now projected to fall in 2009.

5. Against this background, the Government of Mozambique requests access to the Fund's Exogenous Shock Facility (ESF). The Government requests access of 100 percent of quota, or SDR 113.6 million, to be disbursed in thee tranches following completion of the fourth, fifth and sixth reviews under the PSI. In accordance with Fund's policies, the Government is committed to a safeguards assessment to be completed by the time of the first review under the ESF arrangement. 
6. The attached Memorandum of Economic and Financial Policies reviews program implementation during the second half of 2008 and outlines the Government's policies for the remainder of 2009.

7. The Government of Mozambique is confident that the policies set forth in the attached MEFP are adequate to consolidate macroeconomic stability and sustain strong broad-based growth through a second wave of reforms towards the achievement of the Millennium Development Goals. The Government will regularly update the Fund on developments in its economic and financial policies, and provide the data needed for the monitoring of the program. In addition, the Government will consult regularly with the Fund on any relevant developments at the initiative of the Government or the Fund, including for the adoption of new measures, and revisions to the policies described in the MEFP, and provide the Fund with the information it requests for monitoring progress during program implementation.

8. Under the program, the Government has agreed with IMF staff on quantitative ACs and benchmarks for 2009. The Government anticipates that the fifth review, on the basis of program performance to end-June 2009, will be conducted by end-December 2009 and that the sixth review, on the basis of program performance to end-December 2009, will be conducted by end-June 2010. The Government's policies under the program for 2009 are summarized in the attached Memorandum of Economic and Financial Policies (MEFP). The technical memorandum of understanding (TMU) defines the terms and conditions of the policies. The end-June and end-December 2009 ACs, as well as the continuous ACs, will also be performance criteria under the ESF arrangement. Reviews under the ESF arrangement will take place at semi-annual intervals on the occasion of the fifth and sixth PSI reviews.

Sincerely yours,

\author{
/ s / \\ Manuel Chang \\ Minister of Finance
}

\author{
/ s / \\ Ernesto Gouveia Gove \\ Governor \\ Bank of Mozambique
}

Attachments: $\quad$ Memorandum of Economic and Financial Policies

Technical Memorandum of Understanding 


\section{ATtAChMENT I \\ Republic of Mozambique: Memorandum of Economic and Financial Policies of THE GOVERnMENT OF MOZAMBIQUe FOR THE FOURTH REVIEW UNDER THE PSI, AND THE REQUEST FOR ACCESS UNDER THE EXOGENOUS SHOCK FACILITY}

May 13, 2009

1. This MEFP reviews performance under the PSI-supported program through end-2008 and outlines the Government's policies for 2009. The Government of Mozambique is committed to its medium-term macroeconomic strategy of prudent macroeconomic policies and second-generation structural reforms to sustain economic growth and poverty reduction. In the near term, the Government is ready to ease macroeconomic policies to help mitigate the impact of the global economic crisis.

\section{Program Implementation In 2008}

\section{A. Background}

2. Mozambique's macroeconomic performance through 2008 remained strong, despite challenges from the mid-year surge in international fuel and food prices, disruptions to electricity transmission, and the onset of the global economic crisis at the end of the year. The economy expanded by 6.8 percent in 2008, somewhat more than anticipated because of stronger output in transport and communication, mining, and agriculture. Inflation fell faster than expected, from 13 percent in February 2008 to 6.2 percent in December 2008 and 4.4 percent in April 2009, mainly because of the sharp drop in international import prices at the end of the year. As expected, the current account balance in 2008 was adversely affected by higher import expenditures on account of higher international prices. However, this was more than offset by high private capital inflows so that gross external reserves increased about US\$140 million during the year and the real effective exchange rate increased 11 percent.

\section{B. Fiscal Sector and Public Financial Management}

3. The Government's fiscal strategy in 2008 was in line with its medium-term strategy to limit domestic financing to make room for private sector credit. Budget implementation during the year encountered challenges arising from the sharp increase in fuel and food prices.

4. The Government's fiscal plans were revised in July 2008 to incorporate measures to mitigate the social impact of higher fuel and food prices. The Government had already introduced an urban transportation subsidy earlier in the year. In the second half of the year, the Government also suspended fuel-related taxes until 2009 and launched its Food Production Action Plan, together costing 1.3 percent of GDP. These additional measures were largely financed by reductions in nonpriority spending and additional World Bank budget support.

5. Budget implementation in 2008 was strong. Domestic budget financing for the whole year was 0.8 percent of GDP lower than envisaged under the revised fiscal framework. This 
was because of higher domestic revenues ( 0.2 percent of GDP), mainly personal income tax collections; lower recurrent spending mostly ( 0.3 percent of GDP), on goods and services; and other tax revenues ( 0.3 percent). Spending execution was also strong and priority expenditures were 64 percent of total expenditures. However, there was a substantial shortfall in reported project aid of 4.0 percent of GDP.

6. The Revenue Authority (Autoridade Tributária-AT) continued to improve tax administration. It identified strategic reform areas for the year and approved the 2009-2010 Strategic Plan and the 2009 Tactical Plan. To facilitate planning, execution and control of its operations, the AT also started to implement a performance indicator framework. Preparations are under way to allow taxes to be paid through commercial banks by the end of 2009. The AT registered more than 190,000 new tax payers in 2008, some 90 percent more than targeted and contributing substantially to the strong revenue performance.

7. The audit of the stock of post-2003 commercial VAT arrears, completed in July 2008 and covering 80 percent of total invoices, had identified MT 1.3 billion in arrears. The Government is committed to clearing these arrears and the remaining unidentified arrears by end of 2012, starting with a repayment of MT200 million in 2009. The process of reimbursing VAT arrears has improved significantly for current claims and over 90 percent of these claims are now addressed within the time-frame established by regulations.

8. In the area of tax policy, Parliament approved the new Simplified Tax for the Small Tax Payers (ISPC) and the revised Tax Benefits Code in December 2008. Regulations to implement the ISPC were approved in March 2009. Parliament also approved the review of the excise taxes on alcoholic beverages and tobacco (Imposto de Consumo Específico) in January 2009, and these will become effective in January 2010.

9. By March 2009, the e-Sistafe system had been rolled out to 320 state institutions, including 26 autonomous institutions and 50 of the 128 districts. Preparations for the pilot of the electronic revenue collection (e-Tax) were delayed to March 2009, because of financial constraints, and will be completed by the end of 2010. The multi-currency treasury account (CUT) became operational in mid-April for disbursing donor funds in US dollars, euros and the South African rand. Salary payments are carried out through direct budget execution of eSistafe in 21 ministries and other 12 entities at the central level, amounting to 4 percent of all salaries. The 2008 program budgeting pilot, covering five programs in five ministries, was expanded to the entire 2009 budget and covers all ministries and government institutions. Due to the breadth of the consultation process, the approval of the PFM vision paper by the Ministry of Finance was delayed until July (structural benchmark for March 2009). The Government will disseminate the 2007 annual report on public debt on the internet by June. The 2008 public debt report is expected to be finalized and published by July 2009.

10. In May 2009, the Government started implementing the first phase of the medium-term civil service pay policy that was approved by the Council of Ministers in September 2008. The cost is included in the budget and focus on decompressing salary bands. In October 2008, the 
Government published the first statistical yearbook on civil servants, based on the census taken in early 2008.

\section{Monetary and Financial Sector}

11. Monetary policy in 2008 was geared towards price stability in the context of a floating exchange rate regime. The BM met its end-year targets for reserve money and net international reserves. The target for reserve money had been revised to accommodate the projected increase in inflation because of the higher import prices. In the outturn, the BM was able to contain reserve money growth in 2008 below 8 percent, partly because of the strong fiscal performance on domestic financing. In addition, money demand conditions were weaker than expected as import prices and inflation at the end of the year were lower than expected. Money demand conditions were also weaker than anticipated because the BM and banks improved their management of the seasonal surge in currency demand over the festive season.

12. Net international reserves were in line with revised projections, meeting the program target by an ample margin. As anticipated, the World Bank disbursed its budget support for 2009 in late 2008. The BM increased its reserves sales in the second half of 2008 to meet strong import demand pressures, especially for fuel imports. However, import pressures in late 2008 exceeded expectations, putting pressure on banks' own external reserves and also on the exchange rate. After an extended period of stability during much of the year, the exchange rate depreciated 4 percent versus the US dollar in the fourth quarter of 2008 and a further 6 percent in the first quarter of 2009.

13. The monetary program in 2008 was successful in supporting the Government's objective of deepening financial intermediation. Broad money expanded by 20 percent in line with expectations, thereby raising the share of broad money to GDP by one percentage point to about 34 percent of GDP. The stronger-than expected fiscal performance as well as the largerthan-expected decline in the banking sector's net foreign assets made room for a substantial expansion in credit to the economy. This increased 46 percent during 2008 to almost 19 percent of GDP.

14. Lower inflation allowed the BM in February 2009 to reduce the interest rates on its standing lending and deposit facilities. This subsequently led to a decline in the 3-month T-bill rate by about 2 percentage points. The BM in February also reduced the commercial banks' legal reserve requirement from 9 to 8.5 percent.

15. The BM has continued to strengthen its monetary and exchange rate policy and operations. To strengthen policy formulation, the BM has developed its core inflation indicators (structural benchmark for December 2009) and is stepping up its monitoring, analyzing and forecasting of macroeconomic and monetary indicators. To strengthen operations, the BM introduced repos and reverse repos in 2007 and has increasingly used them for market operations. Meticalnet, the money market interbank system, now accommodates outright and repo operations among commercial banks. Strengthening liquidity forecasting and 
management is central to improving monetary operations. It will become particularly important with the passage of donor project aid through the Government's multi-currency treasury account (CUT) at the BM. To support liquidity management, the MF is now providing cash flow projections to the BM on weekly basis and has also started providing projections with a one-year horizon as these are updated. In other areas, the BM has revised the regulations for commercial bank participation in the interbank money market and the guidelines for conduct in the market.

16. An IMF/WB Financial Sector Assessment Program (FSAP) mission assessed the financial sector in February 2009 and found that Mozambique has made significant improvements following the 2003 FSAP. Mozambique had implemented a comprehensive financial sector reform program that has resulted in notable improvements in regulation and supervision. There has been a marked improvement in financial stability and soundness, particularly capital adequacy and asset quality. Nonetheless, due to economy-wide structural impediments to financial intermediation (poor physical access and infrastructure, weaknesses in the legal framework, weak financial reporting, and problems with the credit and property information infrastructure, etc), progress in deepening intermediation has been limited. The global financial crisis has not affected the financial sector directly, but real sector linkages increase credit risk going forward. The system as a whole, however, appears resilient due to strong capital base, high liquidity, constraints on exposure to foreign currency risk by unhedged borrowers, and capital controls. Mozambique has also made commendable progress in improving its national payments system.

\section{Other Structural Policies}

17. To strengthen the management and transparency of mineral resources and to maximize their benefits for Mozambique, all new agreements in mining and petroleum and gas concessions are following the fiscal regulations and agreements that were approved in 2007. Furthermore, the Government submitted its request for Mozambique to become a candidate for adherence to the EITI (structural benchmark for June 2009). The EITI Board approved Mozambique's candidacy on May 18, 2009.

18. Mozambique continues its trade liberalization efforts. The SADC free trade area came into effect in January 2008. In November 2008, Mozambique, together with other SADC members, reached an interim Economic Partnership Agreement (EPA) with the EU to retain quota-and duty-free access to the EU on a reciprocal basis. Agreement on a final EPA is expected by end-2009. Consistent with both this agreement and with the SADC free trade agreement, Parliament approved a reduction of customs tariffs on imports from the EU and SADC countries in December 2008.

19. After extensive consultation, the Ministry of Industry and Commerce submitted in March 2009 a decree to streamline business inspections to the Economic Council. This is expected to be approved by the Council of Ministers by July 2009 (end-March 2009 structural benchmark). In April 2009, the Council of Ministers revoked the bank-deposit and minimum 
capital requirements to start a business. To facilitate the start-up and operations of small and medium enterprises, the Council of Ministers approved the creation of the SME Institute in February 2009. Draft competition and insolvency laws were submitted to Parliament for its approval.

20. The Government has improved its capacity to produce and disseminate macroeconomic statistics, especially at the INE and the MF. The INE has prepared a Statistical Strategic Plan (2008-12). The MF is using e-SISTAFE to generate public finance statistics. However, the public finance statistics are not yet in compliance with the 2001 GFS manual. In order to monitor the impact of the global economic crisis, the Government is compiling macroeconomic data and will start updating its macroeconomic framework on a higher frequency basis. To improve the MF's analytical and forecasting capacity, the MF has started to compile and consolidate data on mega-projects, which will feed into the budget execution reporting.

\section{Program for 2009}

\section{A. Adapting Policies}

21. The Government recognizes that Mozambique's macroeconomic prospects in 2009 have weakened because of the global economic crisis. The Government is nevertheless committed to mitigate the adverse impact of the global crisis, without jeopardizing its medium-term macroeconomic strategy.

22. Against the backdrop of a highly uncertain global economic outlook, economic prospects for 2009 have deteriorated, and GDP growth is now cautiously estimated to 4.3 percent from preliminary 6.8 percent for 2008 . However, the Government intends to make every possible effort to achieve a higher rate of growth. Aluminum and traditional exports receipts are declining due to falling global demand and commodity prices. Project aid disbursements are already being delayed and are expected to fall increasingly short of commitments as fiscal pressures mount in donor countries. Private capital inflows - foreign direct investment and foreign borrowing - which have played an increasingly important role in supporting growth in Mozambique, are contracting as some investors scale back or postpone investment projects in the face of weaker global demand conditions and tighter global credit constraints.

23. The external shock will deteriorate the balance of payments. Net international reserves are projected to fall by over US $\$ 200$ million in 2009 as the trade deficit widens, foreign direct investment contracts, and foreign borrowing by the private sector is curtailed. As a result, the overall balance of payments is projected to deteriorate by nearly US\$400 million between 2008 and 2009. Mitigating factors include a modest projected rise in aid inflows and lower projected dividend payments by megaprojects. Export receipts are expected to fall about US\$800 million, primarily reflecting a halving in the price of aluminum, which accounted for more than half of export receipts in 2008, and lower traditional exports such as cotton and 
cashews. Private capital inflows - foreign direct investment and foreign borrowing - are projected to fall by more than US\$100 million.

24. Notwithstanding this, the impact is expected to be mitigated for Mozambique on account of its sustained period of prudent macroeconomic policies and structural reforms. Moreover, Mozambique will continue to benefit from foreign capital inflows into natural resource areas, especially energy, that remain profitable. In addition, Mozambique's financial sector is largely insulated from and well-positioned to withstand the global financial turmoil. Furthermore, for 2010 the World Cup in South Africa will have favorable impact on the energy and tourism sectors. The more vulnerable segments of the population are expected to benefit from infrastructure investment in agriculture as well from the recent reduction in international fuel and food prices.

25. To mitigate the impact of the global economic slowdown, the Government will ease macroeconomic fiscal and monetary policies during 2009. It will also maintain the momentum of its structural reform agenda for growth, with a particular focus on strengthening the business environment, especially to encourage new investments. Mindful of the risks from the current crisis, the Government will closely monitor international developments and its impact on the Mozambican economy.

26. The Government's scope for supportive macroeconomic policies is curtailed by the large projected decline in external reserves in 2009. The Government estimates that most of these declines are the direct and sudden result of the external shock. On this basis, the Government is seeking access to Fund resources under the Exogenous Shock Facility that will cover a portion of the direct balance of payments impact of the external shock.

\section{B. Fiscal Sector}

27. The Government intends to ease fiscal policy to help the economy weather the global economic slowdown. Mozambique's weaker economic prospects will also deteriorate the fiscal outlook, as revenues are expected to fall well short of previous program targets. The Government will nevertheless maintain domestic spending levels as budgeted. As a result, the Government's domestic financing needs may increase. This is a temporary deviation from the medium-term fiscal strategy as the Government remains committed to avoiding recourse to domestic financing to provide room for private sector credit.

28. Revenues in 2009 are projected to decline by 1.1 percent of GDP compared with the previous program, down to 15.7 percent of GDP. This mainly results from lower corporate income tax receipts from megaprojects and the traditional export sector ( 0.9 percent of GDP), lower fuel taxes ( 0.1 percent of GDP) as global oil prices have declined more sharply than expected, and the delay in increasing excise taxes on tobacco and alcoholic beverages ( 0.1 percent of GDP). The Government will maintain the domestic expenditure envelope in nominal terms broadly as previously envisaged and in line with budgeted levels. Relative to GDP, total domestic spending will rise about $0.3 z$ percent of GDP. As a result, the 
Government may increase domestic financing to about 1.8 percent of GDP, or about 1.1 percent of GDP higher than previously envisaged. Though large, the increase in domestic borrowing does not jeopardize Mozambique's public debt sustainability as the level of public debt is low and increase in domestic financing is expected to be temporary.

29. The Government will be vigilant that the wage bill remains fiscally sustainable, while making room for necessary adjustments to safeguard the social sectors. The wage bill in 2009 will reach 8.9 percent of GDP, the same as previously budgeted and 0.8 percentage points higher than in 2008. Most of the increase (0.4 percent of GDP) will arise as the first pillar of the wage policy reform (the decompression of the salary bands) is implemented. The wage bill also includes additional hiring of 17,500, mostly in the social sectors. Additional reform of the wage policy will be phased in gradually in following years. The MF, in coordination with the Ministry of Civil Service, will prepare an estimate of the new pay policy for the fiscal years 2010 to 2012 by end-August 2009 (structural benchmark).

30. The Government is committed to underpinning sound fiscal policies by strengthening tax administration and public financial management. To modernize the tax administration the AT will further improve its strategic management framework. It will monitor key performance indicators and identify efficiency gains. It will also strengthen the audit and collection enforcement focusing on the mega-projects and strengthening the operational capacity of the Large Tax Payer Units. With the support of IMF and World Bank, the Government intends to make an assessment of its overall tax policy.

31. The Ministry of Finance intends to approve the PFM vision paper for the coming ten years by July (delayed structural benchmark). By September, the Government also intends to approve the Action Plan and Budget for phase III of the PFM reform program which is to be co-funded by donors and Government. The Government will finalize by end-2009 the customization of the e-Tax (e-Tributação) software and complete its pilot implementation by end-2010 (delayed structural benchmark). The Government will issue a decree requiring sectors to receive prior authorization from the MF for any procurement of new accounting systems to ensure that they are complementary and compatible to e-SISTAFE. Finally, the Government will revise its 'Guidelines for External Financing' by end June with the aim of encouraging donors to use the single and multi-currency treasury accounts (CUT) at the BM for disbursing project aid.

32. To strengthen monitoring of net lending to public enterprises, a draft public enterprises law will be submitted to the Council of Ministers by end-September 2009 (structural benchmark). It will include the requirement for quarterly financial reporting to the MF and the ring-fencing of commercial activities.

\section{Monetary Policy and the Financial Sector}

33. The BM is committed to pursuing a prudent monetary policy within the context of a flexible exchange rate regime. Monetary policy will remain geared towards price stability. 
In 2009 , the BM will ease monetary policy modestly to accommodate the expansion in domestic budget financing to limit the adverse impact on credit to the economy. The 12-month inflation at the end of 2009 is expected to remain broadly the same as at the end of 2008 on account of a modest increase in international fuel and food prices and because of the recent depreciation of the exchange rate. Consistent with these objectives, the BM aims to increase reserve money, its operational target, by 12.5 percent during 2009. The weak external environment will have a large adverse impact on Mozambique's external balances. As a result, the BM's gross external reserves are projected to fall US\$221 million during 2009 to US\$1440 at the end of 2009 and equivalent to 3.9 months of imports. The monetary framework makes room for broad money to expand by about 15 percent, allowing for continued financial deepening, including private sector credit growth of 28 percent.

34. The BM will continue reinforcing its monitoring, analysis and forecasting of macroeconomic and monetary indicators. In particular, the BM will continue to focus on strengthening liquidity management, paying particular attention to improving coordination with the MF on its cash flow projections and with banks on their liquidity requirements. Accurate liquidity forecasting will become more challenging as donors shift their project disbursements through the Government's single treasury account (CUT) at the BM. To help strengthen the interbank market and liquidity management, the BM will broaden its use of repos, and build a flexible and more realistic intervention corridor that reflects market conditions. To further strengthen the interbank market, the BM will also stimulate the market's use of repos by improving confidence between the market players as result of the increase of volume and frequency of transactions.

35. The recent FSAP Update found considerable overall progress in the financial sector since the 2003 FSAP. The Update made recommendations in the areas of access to finance, banking supervision, money and debt markets and liquidity management, the nonbank financial sector (pension and insurance), and the payments system. The authorities will finalize an action plan to implement the recommendations by December 2009 and will also form a task force to monitor implementation by the same date. Meanwhile, the BM has already decided to issue new regulations to redefine the loan classification rules to make them consistent with international best practices by September 2009 (structural benchmark). In addition, the BM is preparing improvements in its remedial action framework for banks in difficulty and is strengthening its crisis preparedness and contingency planning with technical assistance from the World Bank and the IMF.

36. The BM will implement measures to further buttress its internal audit. In accordance with the audit charter, the internal audit office will report periodically to the BM's Board on the implementation of audit recommendations and on financial developments at the BM. A new central bank law for Mozambique will be drafted once the SADC central bank model law has been approved (expected in 2010). It will also publish its audited financial statements for 2008 in compliance with IFRS by end-June 2009. 
37. Under the strategic plan (2008-2010), the BM continues to strengthen its banking supervision framework by adopting a risk-based approach to supervise financial institutions. The Risk-Based Supervision (RBS) Framework is expected to be finalized by end-2009. A consultant has been appointed under FSTAP to assist BM on the designing of the road map for the implementation of Basel II in Mozambique. The consultant will assess the impact of Basel II on financial sector and prepare a transition strategy, procedure manuals, and circulars on procedures.

38. The new foreign exchange law which fully liberalizes current account transactions was approved by Parliament in October 2008 and became effective in March 2009. The Government has notified the IMF of the approval of the law and the drafting of its regulations is underway. In the course of 2009, the Government expects to officially inform the Fund of its acceptance of the obligations under Article VIII sections 2, 3, and 4 of the Fund's Articles of Agreement.

\section{Other Structural Policies}

39. Following the recent adoption of a new law on social protection, the Government intends to strengthen the social security and supplementary pension system. Pending the commissioning of a new actuarial study with the World Bank's support, the National Social Security Institute is strengthening the information technology system for its data collection and management systems and will draft and approve by September 2009 an explicit investment strategy. After the census of the civil servants the Government has also finalized the census of pensioners of the civil service pension system and has commissioned an actuarial study with the World Bank's support. Efforts are also underway to strengthen the insurance sector's regulatory and supervisory framework and the institutional capacity of the Inspecção Geral de Seguros.

40. To manage the budgetary and macroeconomic implications of megaprojects, the Government will improve the coordination between MF, MPD, BM, the Institute for National Statistics (INE), and line ministries with the aim to collect and compile data on megaprojects. The Government will strengthen the analytical and forecasting capabilities at the MF, including by strengthening coordination with other Ministries and the BM. The Government will strictly implement the mining and petroleum fiscal regime, related regulations, and model contracts for all new projects. It will also continue to adopt nonrecourse financing clauses in future megaprojects.

41. Following the Government's formal request to become a member of the EITI (structural benchmark for June 2009), the Government, the private sector and civil society will undertake a sequence of actions required for the validation of Mozambique's adherence to the EITI. This process is expected to take at least three years.

42. The Government is intensifying its reforms to improve the business environment. This will help stimulate new investment and mitigate the impact of the global economic slowdown. 
The Government has identified five priority areas. It will: (i) consolidate licensing at the OneStop Shops; (ii) improve the legal framework by implementing the bankruptcy legislation once approved by Parliament, establishing a system for micro-enterprise registration, creating a unified inspection process, and by amending legislation on property registration; (iii) facilitate tax and trade administration by further reducing VAT refund delays, simplifying trade procedures through a Single Window by end-2010, and implementing the e-Tax pilot; (iv) strengthen support for small and medium-sized businesses, by establishing the SME institute and implementing its mandated strategy; and (v) reduce labor market rigidities by disseminating the new labor law and further defining its regulations.

43. The Government places particular emphasis on implementing the second phase of the public sector reform program (2006-11). This consists of four pillars: improving service delivery; strengthening the capacity of local government; creating a more professional public sector; and strengthening good governance systems and the fight against corruption. By the end of 2009, the Government will draft a Public-Private Partnership Law, aimed primarily to prevent and manage fiscal and quasi-fiscal risks, which could also be applicable to megaprojects.

44. The Government took further measures to fight corruption and to improve the efficiency of the public sector. To improve the monitoring, the health sector revised its anticorruption action plan. The ministries of interior, education and culture, finance and justice will finalize theirs by July 2009. The Government has initiated the harmonization and modernization of the national anti-corruption legislation. In 2008, 27 cases were sentenced by the courts for corruption.

45. The Government will prepare a national decentralization strategy by end-December 2009 with the Ministry of State Administration as the coordinator of the process involving other key ministries with the help of the World Bank and other partners. The strategy will include a legal, regulatory, and institutional framework for revenue collection and spending for sub-national units. The strategy will take into account their administrative capacity and need to maintain fiscal control of planning, finance and execution. In the process of reforming the OIIL district revolving funds, the Government has issued in April 2009 instructions to unify the criteria for project selection, lending, and reimbursement rules in the districts.

\section{Program Monitoring}

46. The revised quantitative assessment criteria for end-June 2009 and the new assessment/performance criteria and indicative targets for the second half of 2009 are shown in Table 1. Table 2 lists the structural benchmarks for 2009. 
Table 1. Mozambique: Quantitative Assessment Criteria and Indicative Targets, 2008-09'

(Millions of MT, unless otherwise specified)

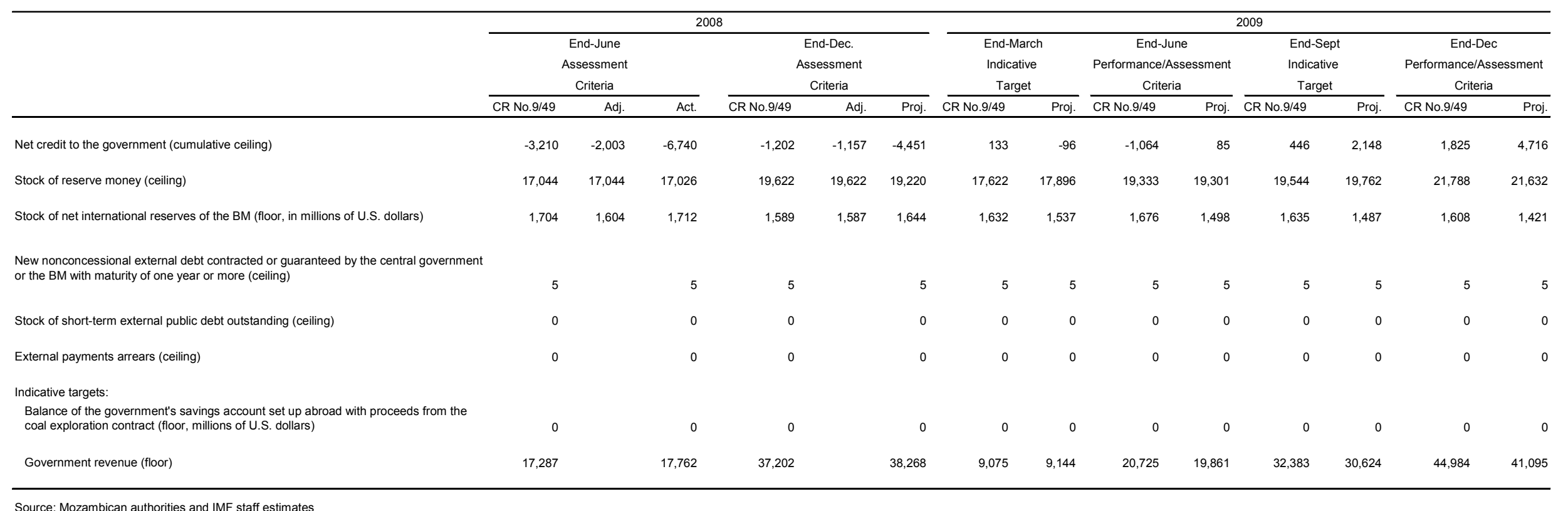

Source: Mozambican authorities and IMF staff estimates

${ }^{1}$ For definition and adjustors see the attached Program Monitoring Section of Memorandum of Economic and Financial Polices and the Technical Memorandum of Understanding. 
Table 2. Mozambique: Structural Benchmarks under the 2009 Policy Supported Instrument (December 2008-December 2009)

\begin{tabular}{|c|c|c|}
\hline Actions & $\begin{array}{l}\text { Expected Date of } \\
\text { Implementation }\end{array}$ & Comments \\
\hline $\begin{array}{l}\text { The Ministry of Finance will approve a PFM vision paper, } \\
\text { as the basis for the Sistafe Action Plan and Budget for } \\
\text { 2009-2011. }\end{array}$ & end-March & $\begin{array}{l}\text { Delayed to } \\
\text { end-July } \\
2009\end{array}$ \\
\hline $\begin{array}{l}\text { The Ministry of Industry and Trade will issue a decree } \\
\text { streamlining the business inspection process. }\end{array}$ & end-March 2009 & $\begin{array}{l}\text { Delayed to } \\
\text { end-July } \\
2009\end{array}$ \\
\hline $\begin{array}{l}\text { The Government will formally submit a request to start the } \\
\text { process to become a member of EITI }\end{array}$ & end-June 2009 & met \\
\hline $\begin{array}{l}\text { The Ministry of Finance in coordination with the Ministry of Public } \\
\text { Service will prepare an estimate of the new pay policy for the fiscal } \\
\text { years 2010-2012. }\end{array}$ & end-August 2009 & $\ldots$ \\
\hline $\begin{array}{l}\text { A draft public enterprises law will be submitted to the } \\
\text { Council of Ministers. }\end{array}$ & $\begin{array}{l}\text { end-September } \\
2009\end{array}$ & $\ldots$ \\
\hline $\begin{array}{l}\text { The central revenue authority and UTRAFE, will } \\
\text { implement the pilot project on corporate income tax for the } \\
\text { revenue collection module (e-Tributação) of the e-Sistafe. }\end{array}$ & $\begin{array}{l}\text { end-September } \\
\qquad 2009\end{array}$ & $\ldots$ \\
\hline $\begin{array}{l}\text { The BM with technical assistance support of the Fund will } \\
\text { design a plan to strengthen the Monetary Policy framework } \\
\text { comprising all intermediate steps, including agreement on a } \\
\text { core inflation indicator. }\end{array}$ & $\begin{array}{l}\text { end-December } \\
2009\end{array}$ & met \\
\hline $\begin{array}{l}\text { The BM will issue new regulations that redefine the loan } \\
\text { classification rules to make them consistent with } \\
\text { international best practices. }\end{array}$ & $\begin{array}{l}\text { end-September } \\
2009\end{array}$ & $\cdots$ \\
\hline
\end{tabular}




\section{ATTACHMENT II \\ RePublic OF Mozambique: Technical Memorandum of Understanding on SELECTED Concepts, Definitions, and DAta Reporting Under MozambiQue's Policy Support INSTRUMENT PROGRAM}

May 13, 2009

1. The purpose of this technical memorandum of understanding (TMU) is to describe the concepts and definitions that will be used in monitoring the Policy Support Instrument (PSI) program, including the following:

- $\quad$ net credit to the central government by the banking system;

- $\quad$ government revenue;

- $\quad$ net international reserves, and reserve money of the Bank of Mozambique (BM);

- new nonconcessional external debt contracted or guaranteed by the central government or the BM with a maturity of more than one year;

- $\quad$ short-term external public debt outstanding;

- $\quad$ external payments arrears; and

- $\quad$ foreign program assistance and external debt service.

This memorandum also describes the adjusters that will be applied to certain quantitative assessment criteria of the program.

\section{Definitions}

\section{Net credit to the central government}

2. Net credit to the central government (NCG) by the banking system is defined as the difference between the outstanding amount of bank credits to the central government and the central government's deposits with the banking system, excluding deposits in project accounts with the banking system, recapitalization bonds issued to the BM, and proceeds from the signing fee for coal exploration. Credits comprise bank loans and advances to the government and holdings of government securities and promissory notes. NCG will be calculated based on data from balance sheets of the monetary authority and commercial banks as per the monetary survey. The limits on the change in net credit to the central government by the banking system will be cumulative from end-December of the previous year.

3. The government encompasses all institutions whose revenue and expenditure are included in the state budget (Orçamento do Estado): central government ministries, agencies, and the administration of 11 provinces. Although local governments (33 municipalities or autarquias) are not included because they are independent, the bulk of their revenue is registered in the state budget as transfers to local governments. 


\section{Government revenue and financing}

4. Revenue is defined to include all receipts of General Directorate of Tax (Direcção Geral dos Impostos or DGI), the General Directorate of Customs (Direcção Geral das Alfândegas, DGA), and nontax revenue, including certain own-generated revenues of districts and some line ministries as defined in the budget. Net receipts from privatization received by the National Directorate of State Assets (Direcção Nacional do Património do Estado) and unrealized profits transferred by the central bank to the treasury will not be considered as revenue (above the line) and will be accounted for as other domestic financing (below the line).

5. For the purpose of program monitoring, revenue is considered as collected at the time when it is received by the DGI from private agents or other government collecting agencies, in cash or checks, or through transfers into a DGI bank account.

\section{Reserve money}

6. Reserve money is defined as the sum of currency issued by BM and the commercial banks' holding at the BM. The reserve money stock will be monitored by the monetary authority and provided to the IMF by the BM.

\section{Net international reserves}

7. Net international reserves of the BM are defined as reserve assets minus reserve liabilities. The BM's reserve assets include (a) monetary gold; (b) holdings of SDRs; (c) reserve position at the IMF; (d) holdings of foreign exchange; and (e) claims on nonresidents, such as deposits abroad (excluding the government's savings account related to the Moatize coal mine concession). Reserve assets exclude assets pledged or otherwise encumbered, including but not limited to assets used as collateral or guarantee for a third-party external liability (assets not readily available.) The BM's reserve liabilities include (a) all shortterm foreign exchange liabilities to nonresidents with original maturity of up to and including one year; and (b) all liabilities to the IMF.

8. For the purposes of program monitoring and in order to exclude valuation changes, net international reserves will be measured at the official exchange rates prevailing at end-2007 and used by the BM.

9. The BM will publish the exchange rates quoted by commercial banks on average as the market rates.

\section{New nonconcessional external debt contracted or guaranteed by the central government or the BM with maturity of more than one year}

10. The term 'debt' will have the meaning set forth in Point 9 of the Guidelines on Performance Criteria with Respect to Foreign Debt adopted on August 24, 2000. Government debt is outstanding debt owed or guaranteed by the central government or the BM (but does not 
include debt of any political subdivision or government-owned entity with a separate legal personality that is not otherwise owed or guaranteed by the central government).

11. The central government will limit contracting or guaranteeing external debt up to US\$5 million each year with original maturity of one year or more with a grant element of less than 35 percent, calculated using currency-specific discount rates based on the Organization for Economic Cooperation and Development (OECD) commercial interest reference rates in accordance with the annual Budget Law. This assessment criterion applies not only to debt as defined in point 9 of the Guidelines on Performance Criteria with Respect to Foreign Debt adopted on August 24, 2000, but also to commitments contracted or guaranteed for which value has not been received. This assessment criterion will be assessed on a continuous basis.

\section{Stock of short-term external public debt outstanding}

12. The central government will not contract or guarantee external debt with original maturity of less than one year. This assessment criterion applies not only to debt as defined in point 9 of the Guidelines on Performance Criteria with Respect to Foreign Debt adopted on August 24, 2000, but also to commitments contracted or guaranteed for which value has not been received. Excluded from this assessment criterion are short-term, import-related trade credits. This assessment criterion will be assessed on a continuous basis.

\section{External payments arrears}

13. The government undertakes not to incur payments arrears on external debt owed or guaranteed by the central government, with the exception of external payments arrears arising from government debt that is being renegotiated with creditors, including Paris Club creditors. This assessment criterion will be assessed on a continuous basis.

\section{Foreign program assistance}

14. Foreign program assistance is defined as grants and loans received by the Ministry of Finance through BM accounts excluding those related to projects (Table 1).

\section{Actual external debt-service payments}

15. Actual external debt-service payments are defined as cash payments on external debtservice obligations of the government and central bank, including obligations to Paris Club and other bilateral creditors rescheduled under enhanced HIPC Initiative completion point terms, multilateral creditors, and private creditors, but excluding obligations to the IMF (Table 1). 


\section{Adjusters}

\section{Net international reserves}

16. The quantitative targets (floors) for the central bank's net international reserves will not be adjusted for any excess in disbursements of foreign program assistance (and any shortfall in external debt service), compared to the program baseline. These targets will be adjusted downward by 100 percent of any shortfall in external program aid (up to a maximum of US\$100 million) and any excess in external debt service payments, compared to the program baseline (Table 1).

17. The quantitative targets (floors) for the central bank's net international reserves will be adjusted downward/upward for any revision made to the end-year figures corresponding to the previous year.

18. The quantitative targets (floors) for the central bank's net international reserves will be adjusted upward for the full amount of any external privatization proceeds in excess of those envisaged in the program, unless these proceeds are deposited in the government's savings account abroad. If they are deposited abroad, the indicative targets on the balance in this account will be adjusted upward for the full amount of the higher than envisaged proceeds, net of any costs related to the privatizations, including severance payments.

19. The quantitative targets (floors) for the central bank's net international reserves will be adjusted downward up to a maximum of US\$50 million per annum by the amount that the actual fuel import bill is higher due to a rise in the average fuel import price. This adjustment will be equal to the difference between the realized and the programmed average fuel import price, multiplied by the volume of total fuel imports programmed for each quarter (Table 1).

20. The quantitative target (floor) on net international reserves will be adjusted downward to accommodate the possible need for higher external outlays to deal with natural disasters, up to a total limit of US\$20 million.

21. In case that Mozambique participates in any SDR allocation(s) between June 30, 2009 and the test date, and provided that SDR allocation(s) are not included as part of reserve liabilities for the purpose of calculating NIR, then the quantitative target (floor) for NIR will be adjusted upwards by the full equivalent of the amount of the cumulative additional SDR allocation(s) up to the test date, measured at program exchange rates.

\section{Net credit to government}

22. The quantitative targets (ceilings) for net credit on the central government (NCG) will not be adjusted for any excess in disbursements of foreign program assistance (and any shortfall in external debt service), compared to the program baseline. These targets will be adjusted upward by 100 percent of any shortfall in external program aid (up to a maximum of US $\$ 100$ million) and any excess in external debt service payments, compared to the program baseline (Table 1). 
23. The quantitative targets (ceilings) for net credit on the central government (NCG) will be adjusted downward for the full amount of any privatization proceeds in excess of those envisaged in the program, unless these proceeds are deposited in the government's savings account abroad.

24. The quantitative targets (ceilings) for net credit on the central government (NCG) will be adjusted downward (upward) for any increase (decrease) in domestic financing from the nonfinancial private sector. The government expenditures contingent on higher disbursements of foreign program assistance (and lower external debt service) will be used to finance greater 'priority' spending identified in the budget.

25. The quantitative target (ceiling) for net credit to the central government (NCG) will be adjusted upward to accommodate the possible need for higher locally financed government outlays to deal with natural disasters, up to a total limit of MT 500 million.

\section{Reserve money}

26. The quantitative target (ceiling) for reserve money will be adjusted upward up to a maximum of MT 750 million to the extent that the sum of the outstanding stock of currency issued by the BM exceed those projected in Table 1. The maximum at end-December will be raised to MT 1000 million because of the seasonal increase in the demand for currency during the end-year festive season.

\section{Data Reporting}

27. In addition to providing the monthly and quarterly data needed to monitor program implementation in relation to the programs' quantitative targets and broader economic developments, the authorities will provide weekly updates of the daily data set out in Table 1 as well as the weekly data set out in Table 4 of the TMU dated May 26, 2005. Monthly updates will also be provided of the foreign exchange cash flow of the BM.

28. The government will continue to provide Fund staff with the data corresponding to monthly government revenues (in detail according to the fiscal table), with a lag not exceeding one month. In addition, the government will continue to publish and provide Fund staff with the quarterly budget execution reports with a time lag not exceeding 45 days.

29. In addition, the government will provide monthly information on the balance of its savings account abroad and will start developing and providing information on domestic arrears on a quarterly basis.

30. From December 2005 onwards, the monetary survey made available by the BM will clearly identify donor-financed project deposits (with a breakdown between foreign and domestic currency) included in net credit to the government in both the central bank's and commercial banks' balance sheets. 
Table 1. Mozambique: Technical Memorandum of Understanding, 2008-09

\begin{tabular}{|c|c|c|c|c|c|c|c|c|c|c|c|c|}
\hline & \multirow{2}{*}{\multicolumn{2}{|c|}{$\begin{array}{l}2008 \\
\text { Year }\end{array}$}} & \multicolumn{10}{|c|}{2009} \\
\hline & & & \multicolumn{2}{|l|}{ Q1 } & \multicolumn{2}{|c|}{ Q2 } & \multicolumn{2}{|c|}{ Q3 } & \multicolumn{2}{|c|}{ Q4 } & \multicolumn{2}{|c|}{ Year } \\
\hline & CR No.9/49 & Act. & CR No.9/49 & Proj. & CR No.9/49 & Rev. Prog. & CR No.9/49 & Rev. Prog. & CR No.9/49 & Rev. Prog. & CR No.9/49 & Rev. Prog. \\
\hline Net foreign program assistance (US\$ mn) & 501 & 502 & 153 & 105 & 105 & 105 & 42 & 78 & 55 & 68 & 356 & 356 \\
\hline Gross foreign program assistance & 552 & 550 & 164 & 116 & 117 & 117 & 63 & 98 & 69 & 82 & 413 & 413 \\
\hline Program grants & 371 & 371 & 164 & 116 & 117 & 117 & 63 & 83 & 39 & 67 & 382 & 382 \\
\hline Program loans & 182 & 178 & 0 & 0 & 0 & 0 & 0 & 15 & 31 & 15 & 31 & 30 \\
\hline External debt service & 52 & 48 & 11 & 11 & 11 & 11 & 21 & 21 & 14 & 14 & 57 & 57 \\
\hline Net foreign program assistance (MT $\mathrm{mn}$ ) & 11,950 & 11,934 & $3,776.16$ & 2,735 & 2,338 & 2,643 & 1,203 & 2,434 & 1,258 & 1,740 & 8,575 & 9,552 \\
\hline Gross foreign program assistance & 13,330 & 13,284 & $4,054.71$ & 3,003 & 2,905 & 3,293 & 1,574 & 2,852 & 1,771 & 2,366 & 10,305 & 11,514 \\
\hline Program grants & 8,923 & 8,949 & $4,054.71$ & 3,003 & 2,905 & 3,293 & 1,574 & 2,412 & 990 & 1,922 & 9,523 & 10,632 \\
\hline Program loans & 4,407 & 4,335 & 0.00 & 0 & 0 & 0 & 0 & 439 & 782 & 443 & 782 & 883 \\
\hline External debt service & 1,380 & 1,351 & 278.55 & 268 & 567 & 650 & 371 & 418 & 513 & 626 & 1,730 & 1,962 \\
\hline Stock of outstanding currency (MT bm) & 12,036 & 11,996 & 10,075 & 10,587 & 11,323 & 11,677 & 11,383 & 11,857 & 13,000 & 12,763 & 13,000 & 12,763 \\
\hline Average global oil price, APSP, US $\$ /$ barrel & 107 & 97 & 72 & 44 & 74 & 52 & 76 & 55 & 78 & 58 & 75 & 52 \\
\hline Average fuel import price, US $\$ /$ barrel (10 barrels per mt) & 102 & 115 & 68 & 131 & 67 & 117 & 55 & 100 & 70 & 81 & 65 & 109 \\
\hline Volume of fuel imports (1000s mt) & 620 & 549 & 154 & 188 & 133 & 228 & 202 & 165 & 280 & 154 & 770 & 736 \\
\hline Total fuel import bill (US\$m) & 635 & 632 & 105 & 247 & 89 & 266 & 110 & 165 & 196 & 125 & 500 & 803 \\
\hline Cumulative fuel import bill (US\$m) & 635 & 632 & 105 & 247 & 194 & 513 & 304 & 678 & 500 & 803 & 500 & 803 \\
\hline
\end{tabular}

Source: Mozambican authorities and IMF staff estimates 


\section{APPENDIX II \\ REPUblic OF MoZaMbiQUe: DebT SUSTAINABILITY-UPDATE}

\section{In view of Mozambique's request for access to Fund resources under the Exogenous} Shocks Facility, Fund staff have updated the assessment of Mozambique's debt sustainability.

The previous assessment found that Mozambique's debt indicators were expected to remain well below their thresholds and that the risk of debt distress was low. This assessment builds on the joint Fund/World Bank assessment presented to the respective Executive Boards at the time of the $3^{\text {rd }}$ PSI review. It incorporates the previous projections of debt service on the stock of outstanding external debt and incorporates debt service projections on new domestic and external debt obligations, including the proposed use of Fund resources. The macroeconomic assumptions underlying the assessment have also been revised.

2. In staff's view, Mozambique continues to face a low of risk of debt distress, though its public debt sustainability ratios have somewhat deteriorated. This is largely because of the deterioration in the sustainability ratios for external debt, that constitute the bulk of Mozambique's public debt. Mozambique's external debt and debt service are projected to rise relative to GDP, exports and revenues during 2009 and beyond, mostly because of lower nominal GDP, export receipts, and revenues measured in US dollars. The use of Fund resources will have only a modest impact on the ratios. With the proposed use of Fund resource, the debt indicators continue to remain below their thresholds. 
Table 1.: External Debt Sustainability Framework, Baseline Scenario, 2006-2029 1

(In percent of GDP, unless otherwise indicated)

\begin{tabular}{|c|c|c|c|c|c|c|c|c|c|c|c|c|c|c|c|}
\hline & \multicolumn{3}{|c|}{ Actual } & \multirow{2}{*}{$\begin{array}{cc}\text { Historical } & 0 \\
\text { Average } & 0\end{array}$} & \multirow{2}{*}{$\begin{array}{c}\text { Standard } \\
\text { Deviation }\end{array}$} & \multicolumn{6}{|c|}{ Projections } & \multirow[b]{2}{*}{$2009-2014$} & & & \multirow{2}{*}{$\begin{array}{r}2015-202 \\
\text { Average } \\
\end{array}$} \\
\hline & 2006 & 2007 & 2008 & & & 2009 & 2010 & 2011 & 2012 & 2013 & 2014 & & 2019 & 2029 & \\
\hline External debt (nominal) 1/ & 66.6 & 55.8 & 36.8 & & & 44.7 & 48.3 & 50.4 & 51.4 & 52.2 & 53.0 & & 51.1 & 45.0 & \\
\hline $\mathrm{o} / \mathrm{w}$ public and publicly guaranteed $(\mathrm{PPG})$ & 47.1 & 38.1 & 22.5 & & & 30.4 & 35.1 & 38.0 & 39.7 & 40.8 & 41.5 & & 39.1 & 29.8 & \\
\hline Identified net debt-creating flows & -30.6 & -2.8 & -6.3 & & & 3.8 & 1.4 & -1.4 & -4.1 & -2.5 & -1.4 & & -1.5 & 1.2 & \\
\hline Non-interest current account deficit & -22.4 & 7.9 & 7.3 & 3.1 & 9.7 & 8.3 & 6.8 & 5.3 & 3.1 & 4.9 & 6.1 & & 5.8 & 8.8 & 6.4 \\
\hline Deficit in balance of goods and services & 7.4 & 10.7 & 12.4 & & & 20.6 & 13.6 & 13.5 & 13.9 & 14.3 & 14.5 & & 13.4 & 13.3 & \\
\hline Exports & 39.7 & 36.9 & 33.3 & & & 26.6 & 31.1 & 32.3 & 32.0 & 31.2 & 30.8 & & 32.5 & 42.8 & \\
\hline Imports & 47.1 & 47.6 & 45.7 & & & 47.3 & 44.6 & 45.7 & 45.9 & 45.5 & 45.4 & & 45.9 & 56.1 & \\
\hline Net current transfers (negative $=$ inflow) & -35.6 & -9.8 & -10.6 & -12.6 & 8.3 & -12.0 & -14.1 & -16.1 & -18.7 & -16.9 & -15.6 & & -14.7 & -14.0 & -15.1 \\
\hline $\mathrm{o} / \mathrm{w}$ official & -35.6 & -9.8 & -10.6 & & & -12.0 & -14.1 & -16.1 & -18.7 & -16.9 & -15.6 & & -14.7 & -14.0 & \\
\hline Other current account flows (negative $=$ net inflow) & 5.9 & 7.0 & 5.5 & & & -0.3 & 7.3 & 7.9 & 7.9 & 7.5 & 7.1 & & 7.0 & 9.4 & \\
\hline Net FDI (negative $=$ inflow) & -2.1 & -5.3 & -5.9 & -5.3 & 2.4 & -5.6 & -5.6 & -5.9 & -5.9 & -5.9 & -5.9 & & -5.9 & -5.9 & -5.9 \\
\hline Endogenous debt dynamics $2 /$ & -6.1 & -5.5 & -7.7 & & & 1.0 & 0.2 & -0.8 & -1.2 & -1.5 & -1.6 & & -1.4 & -1.7 & \\
\hline Contribution from nominal interest rate & 2.3 & 1.8 & 2.4 & & & 2.7 & 2.4 & 1.9 & 1.7 & 1.7 & 1.7 & & 1.8 & 1.2 & \\
\hline Contribution from real GDP growth & -7.5 & -4.4 & -3.1 & & & -1.7 & -2.2 & -2.7 & -2.9 & -3.2 & -3.3 & & -3.2 & -2.9 & \\
\hline Contribution from price and exchange rate changes & -0.9 & -2.9 & -7.0 & & & $\ldots$ & & & $\ldots$ & 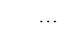 & $\ldots$ & & & & \\
\hline Residual (3-4) 3/ & 2.3 & -8.0 & -12.6 & & & 4.1 & 2.2 & 3.5 & 5.1 & 3.3 & 2.2 & & 0.8 & -3.0 & \\
\hline $\mathrm{o} / \mathrm{w}$ exceptional financing & 0.0 & 0.0 & 0.0 & & & 0.0 & 0.0 & 0.0 & 0.0 & 0.0 & 0.0 & & 0.0 & 0.0 & \\
\hline PV of external debt $4 /$ & $\ldots$ & $\ldots$ & 24.6 & & & 29.3 & 31.0 & 32.1 & 32.6 & 33.2 & 33.9 & & 32.9 & 31.8 & \\
\hline In percent of exports & & $\ldots$ & 73.9 & & & 109.8 & 99.9 & 99.5 & 101.8 & 106.4 & 110.0 & & 101.5 & 74.4 & \\
\hline PV of PPG external debt & $\ldots$ & $\ldots$ & 10.3 & & & 15.0 & 17.9 & 19.7 & 20.9 & 21.7 & 22.4 & & 21.0 & 16.7 & \\
\hline In percent of government revenues & $\ldots$ & $\ldots$ & 64.1 & & & 95.0 & 111.4 & 119.3 & 124.7 & 126.6 & 128.9 & & 115.4 & 65.4 & \\
\hline Debt service-to-exports ratio (in percent) & 14.1 & 42.9 & 52.0 & & & 27.8 & 23.3 & 21.4 & 20.6 & 19.5 & 19.9 & & 18.6 & 14.8 & \\
\hline PPG debt service-to-exports ratio (in percent) & 2.6 & 1.6 & 33.2 & & & 1.6 & 1.5 & 1.7 & 2.1 & 2.2 & 2.5 & & 4.2 & 3.9 & \\
\hline PPG debt service-to-revenue ratio (in percent) & 6.8 & 3.7 & 69.1 & & & 2.8 & 3.0 & 3.4 & 4.1 & 4.0 & 4.4 & & 7.5 & 6.5 & \\
\hline Total gross financing need (Billions of U.S. dollars) & -1.4 & 1.5 & 1.8 & & & 0.9 & 0.8 & 0.6 & 0.4 & 0.6 & 0.8 & & 1.0 & 2.5 & \\
\hline Non-interest current account deficit that stabilizes debt ratio & 5.9 & 18.7 & 26.2 & & & 0.5 & 3.2 & 3.2 & 2.1 & 4.2 & 5.3 & & 6.5 & 10.6 & \\
\hline \multicolumn{16}{|l|}{ Key macroeconomic assumptions } \\
\hline Real GDP growth (in percent) & 8.7 & 7.4 & 6.8 & 7.7 & 2.4 & 4.3 & 5.0 & 6.0 & 6.2 & 6.7 & 6.7 & 5.8 & 6.6 & 6.5 & 6.5 \\
\hline GDP deflator in US dollar terms (change in percent) & 0.9 & 4.6 & 14.4 & 1.3 & 9.2 & -9.7 & -1.8 & 1.5 & 1.7 & 1.6 & -0.5 & -1.2 & -0.2 & -2.2 & -1.2 \\
\hline Effective interest rate (percent) 5/ & 2.6 & 3.1 & 5.3 & 3.2 & 0.9 & 6.9 & 5.5 & 4.2 & 3.6 & 3.5 & 3.5 & 4.5 & 3.8 & 2.8 & 3.5 \\
\hline Growth of exports of G\&S (US dollar terms, in percent) & 34.0 & 4.3 & 10.2 & 20.6 & 12.0 & -24.7 & 20.2 & 11.7 & 7.2 & 5.6 & 5.1 & 4.2 & 7.7 & 7.4 & 7.5 \\
\hline Growth of imports of G\&S (US dollar terms, in percent) & 18.4 & 13.6 & 17.1 & 14.0 & 10.0 & -2.5 & -2.6 & 10.2 & 8.3 & 7.6 & 5.9 & 4.5 & 6.8 & 6.6 & 6.6 \\
\hline Grant element of new public sector borrowing (in percent) & $\ldots$ & $\ldots$ & $\ldots$ & $\ldots$ & $\ldots$ & 42.5 & 47.4 & 48.9 & 50.2 & 50.4 & 50.4 & 48.3 & 50.2 & 49.1 & 49.8 \\
\hline Government revenues (excluding grants, in percent of GDP) & 15.0 & 15.9 & 16.0 & & & 15.7 & 16.0 & 16.5 & 16.7 & 17.2 & 17.4 & & 18.2 & 25.6 & 20.4 \\
\hline Aid flows (in Billions of US dollars) $7 /$ & 0.8 & 1.0 & 1.2 & & & 1.4 & 1.8 & 2.1 & 1.8 & 1.9 & 1.8 & & 1.9 & 1.9 & \\
\hline $\mathrm{o} / \mathrm{w}$ Grants & 0.6 & 0.8 & 0.9 & & & 1.1 & 1.3 & 1.7 & 1.4 & 1.5 & 1.4 & & 1.4 & 1.4 & \\
\hline $\mathrm{o} / \mathrm{w}$ Concessional loans & 0.2 & 0.2 & 0.2 & & & 0.3 & 0.4 & 0.4 & 0.4 & 0.4 & 0.4 & & 0.4 & 0.4 & \\
\hline Grant-equivalent financing (in percent of GDP) 8 / & $\ldots$ & $\ldots$ & $\ldots$ & & & 14.7 & 17.1 & 18.8 & 14.9 & 14.2 & 13.2 & & 9.7 & 6.3 & 8.7 \\
\hline Grant-equivalent financing (in percent of external financing) $8 /$ & $\ldots$ & $\ldots$ & $\ldots$ & & & 79.0 & 83.5 & 87.1 & 86.2 & 86.8 & 86.4 & & 86.0 & 85.8 & 85.9 \\
\hline \multicolumn{16}{|l|}{ Memorandum items: } \\
\hline Nominal GDP (Billions of US dollars) & 7.2 & 8.1 & 9.9 & & & 9.3 & 9.6 & 10.3 & 11.2 & 12.1 & 12.9 & & 17.7 & 27.3 & \\
\hline Nominal dollar GDP growth & 9.7 & 12.3 & 22.2 & & & -5.9 & 3.1 & 7.5 & 8.0 & 8.5 & 6.2 & 4.6 & 6.4 & 4.2 & 5.2 \\
\hline
\end{tabular}

\section{Sources: Country authorities; and staff estimates and projections.}

$1 /$ Includes both public and private sector external debt.

2/ Derived as $[\mathrm{r}-\mathrm{g}-\rho(1+\mathrm{g})](1+\mathrm{g}+\rho+\mathrm{g} \rho)$ times previous period debt ratio, with $\mathrm{r}=$ nominal interest rate; $\mathrm{g}=$ real GDP growth rate, and $\rho=$ growth rate of GDP deflator in U.S. dollar terms.

3/ Includes exceptional financing (i.e., changes in arrears and debt relief); changes in gross foreign assets; and valuation adjustments. For projections also includes contribution from price and exchange rate changes.

5/ Current-year interest payments divided by previous period debt stock.

6/ Historical averages and standard deviations are generally derived over the past 10 years, subject to data availability.
f

7/ Defined as grants, concessional loans, and debt relief.

8 / Grant-equivalent financing includes grants provided directly to the government and through new borrowing (difference between the face value and the PV of new debt). 
Table 2.Mozambique: Public Sector Debt Sustainability Framework, Baseline Scenario, 2006-2029

(In percent of GDP, unless otherwise indicated)

\begin{tabular}{|c|c|c|c|c|c|c|c|c|c|c|c|c|c|c|c|}
\hline & \multicolumn{3}{|c|}{ Actual } & \multirow[b]{2}{*}{ Average } & \multirow[b]{2}{*}{$\begin{array}{c}\text { Standard } \\
\text { Deviation } \\
\end{array}$} & \multicolumn{5}{|l|}{ Estimate } & \multicolumn{5}{|c|}{ Projections } \\
\hline & 2006 & 2007 & 2008 & & & 2009 & 2010 & 2011 & 2012 & 2013 & 2014 & $\begin{array}{l}2009-14 \\
\text { Average } \\
\end{array}$ & 2019 & 2029 & $\begin{array}{l}2015-29 \\
\text { Average } \\
\end{array}$ \\
\hline Public sector debt 1 / & 53.4 & 41.9 & 24.7 & & & 33.1 & 37.6 & 40.1 & 41.6 & 42.4 & 42.9 & & 39.9 & 30.2 & \\
\hline $\mathrm{o} / \mathrm{w}$ foreign-currency denominated & 47.1 & 38.1 & 22.5 & & & 30.4 & 35.1 & 38.0 & 39.7 & 40.8 & 41.5 & & 39.1 & 29.8 & \\
\hline Change in public sector debt & -27.5 & -11.5 & -17.2 & & & 8.4 & 4.4 & 2.6 & 1.4 & 0.8 & 0.6 & & -1.0 & -1.2 & \\
\hline Identified debt-creating flows & -5.2 & -7.9 & -0.8 & & & 5.9 & 2.5 & 1.2 & 2.7 & 1.9 & 0.9 & & 2.1 & 1.1 & \\
\hline Primary deficit & 3.2 & 2.3 & 1.9 & 1.8 & 2.6 & 4.9 & 3.7 & 3.3 & 5.1 & 4.5 & 3.6 & 4.2 & 4.5 & 2.6 & 4.4 \\
\hline Revenue and grants & 22.9 & 25.2 & 25.5 & & & 27.6 & 30.1 & 32.6 & 29.3 & 29.2 & 28.5 & & 26.3 & 30.8 & \\
\hline of which: grants & 7.9 & 9.3 & 9.5 & & & 11.8 & 14.0 & 16.1 & 12.5 & 12.0 & 11.1 & & 8.1 & 5.3 & \\
\hline Primary (noninterest) expenditure & 26.2 & 27.5 & 27.4 & & & 32.5 & 33.8 & 35.9 & 34.4 & 33.7 & 32.1 & & 30.8 & 33.4 & \\
\hline Automatic debt dynamics & -7.4 & -9.9 & -2.6 & & & 1.5 & -1.2 & -2.0 & -2.5 & -2.6 & -2.7 & & -2.5 & -1.5 & \\
\hline Contribution from interest rate/growth differential & -6.8 & -5.4 & -6.9 & & & 1.8 & -0.6 & -2.1 & -2.4 & -2.7 & -1.9 & & -1.8 & -0.9 & \\
\hline of which: contribution from average real interest rate & -0.3 & -1.7 & -4.2 & & & 2.9 & 1.0 & 0.0 & 0.0 & 0.0 & 0.7 & & 0.8 & 1.0 & \\
\hline of which: contribution from real GDP growth & -6.5 & -3.7 & -2.7 & & & -1.0 & -1.6 & -2.1 & -2.3 & -2.6 & -2.7 & & -2.5 & -1.9 & \\
\hline Contribution from real exchange rate depreciation & -0.6 & -4.5 & 4.3 & & & -0.4 & -0.7 & 0.1 & -0.1 & 0.1 & -0.7 & & & & \\
\hline Other identified debt-creating flows & -1.1 & -0.3 & -0.1 & & & -0.5 & 0.0 & 0.0 & 0.0 & 0.0 & 0.0 & & 0.0 & 0.0 & \\
\hline Privatization receipts (negative) & 0.0 & 0.0 & 0.0 & & & -0.4 & 0.0 & 0.0 & 0.0 & 0.0 & 0.0 & & 0.0 & 0.0 & \\
\hline Recognition of implicit or contingent liabilities & 0.0 & 0.0 & 0.0 & & & 0.0 & 0.0 & 0.0 & 0.0 & 0.0 & 0.0 & & 0.0 & 0.0 & \\
\hline Debt relief (HIPC and other) & -1.1 & -0.3 & -0.1 & & & -0.1 & 0.0 & 0.0 & 0.0 & 0.0 & 0.0 & & 0.0 & 0.0 & \\
\hline Other (specify, e.g. bank recapitalization) & 0.0 & 0.0 & 0.0 & & & 0.0 & 0.0 & 0.0 & 0.0 & 0.0 & 0.0 & & 0.0 & 0.0 & \\
\hline Residual, including asset changes & -22.3 & -3.6 & -16.4 & & & 2.5 & 1.9 & 1.3 & -1.3 & -1.1 & -0.3 & & -3.0 & -2.4 & \\
\hline \multicolumn{16}{|l|}{ Other Sustainability Indicators } \\
\hline PV of public sector debt & 6.3 & 3.9 & 12.5 & & & 17.7 & 20.3 & 21.8 & 22.7 & 23.3 & 23.8 & & 21.8 & 17.0 & \\
\hline $\mathrm{o} / \mathrm{w}$ foreign-currency denominated & 0.0 & 0.0 & 10.3 & & & 15.0 & 17.9 & 19.7 & 20.9 & 21.7 & 22.4 & & 21.0 & 16.7 & \\
\hline $\mathrm{o} / \mathrm{w}$ external & $\ldots$ & $\ldots$ & 10.3 & & & 15.0 & 17.9 & 19.7 & 20.9 & 21.7 & 22.4 & & 21.0 & 16.7 & \\
\hline $\mathrm{PV}$ of contingent liabilities (not included in public sector debt) & & $\ldots$ & $\ldots$ & & & $\ldots$ & $\ldots$ & $\ldots$ & & $\ldots$ & $\ldots$ & & $\ldots$ & $\ldots$ & \\
\hline Gross financing need $2 /$ & 7.6 & 5.7 & 13.6 & & & 5.8 & 4.6 & 4.2 & 6.2 & 5.5 & 4.6 & & 6.1 & 4.4 & \\
\hline $\mathrm{PV}$ of public sector debt-to-revenue and grants ratio (in percent) & 27.6 & 15.4 & 49.1 & & & 64.3 & 67.4 & 66.9 & 77.7 & 79.9 & 83.6 & & 83.0 & 55.2 & \\
\hline $\mathrm{PV}$ of public sector debt-to-revenue ratio (in percent) & 42.2 & 24.3 & 78.1 & & & 112.5 & 126.4 & 132.1 & 135.9 & 136.0 & 137.1 & & 119.8 & 66.6 & \\
\hline $\mathrm{o} / \mathrm{w}$ external $3 /$ & $\ldots$ & $\ldots$ & 64.1 & & & 95.0 & 111.4 & 119.3 & 124.7 & 126.6 & 128.9 & & 115.4 & 65.4 & \\
\hline Debt service-to-revenue and grants ratio (in percent) 4/ & 7.6 & 4.9 & 45.6 & & & 2.9 & 3.1 & 3.0 & 3.6 & 3.5 & 3.7 & & 5.9 & 5.8 & \\
\hline Debt service-to-revenue ratio (in percent) 4/ & 11.6 & 7.8 & 72.6 & & & 5.1 & 5.8 & 5.9 & 6.3 & 6.0 & 6.0 & & 8.6 & 7.0 & \\
\hline Primary deficit that stabilizes the debt-to-GDP ratio & 30.8 & 13.9 & 19.1 & & & -3.5 & -0.7 & 0.7 & 3.7 & 3.6 & 3.0 & & 5.5 & 3.9 & \\
\hline \multicolumn{16}{|l|}{ Key macroeconomic and fiscal assumptions } \\
\hline Real GDP growth (in percent) & 8.7 & 7.4 & 6.8 & 7.7 & 2.4 & 4.3 & 5.0 & 6.0 & 6.2 & 6.7 & 6.7 & 5.8 & 6.6 & 6.5 & 6.5 \\
\hline Average nominal interest rate on forex debt (in percent) & 0.4 & 0.5 & 0.7 & 1.2 & 1.1 & 1.1 & 0.9 & 1.0 & 1.0 & 1.1 & 1.1 & 1.0 & 1.6 & 1.1 & 1.3 \\
\hline Average real interest rate on domestic debt (in percent) & 0.4 & 0.1 & 2.3 & 11.7 & 13.1 & 12.6 & 8.7 & 9.0 & 9.5 & 9.6 & 9.1 & 9.8 & 8.8 & 11.6 & 10.0 \\
\hline Real exchange rate depreciation (in percent,+ indicates depreciation) & -0.9 & -10.7 & 13.7 & 0.4 & 13.8 & -1.5 & $\ldots$ & $\ldots$ & $\ldots$ & $\ldots$ & $\ldots$ & $\ldots$ & $\ldots$ & $\ldots$ & $\ldots$ \\
\hline Inflation rate (GDP deflator, in percent) & 9.3 & 7.5 & 7.7 & 8.4 & 2.9 & 4.6 & 5.0 & 5.0 & 5.0 & 4.8 & 4.8 & 4.9 & 5.0 & 3.0 & 4.0 \\
\hline Growth of real primary spending (deflated by GDP deflator, in percent) & 0.3 & 0.1 & 0.1 & 0.1 & 0.2 & 0.2 & 0.1 & 0.1 & 0.0 & 0.0 & 0.0 & 0.1 & 0.1 & 0.1 & 0.1 \\
\hline Grant element of new external borrowing (in percent) & $\ldots$ & $\ldots$ & $\ldots$ & ... & ... & 42.5 & 47.4 & 48.9 & 50.2 & 50.4 & 50.4 & 48.3 & 50.2 & 49.1 & ... \\
\hline
\end{tabular}

Sources: Country authorities; and staffe estimates and projections.

1/ [Indicate coverage of public sector, e.g., general government or nonfinancial public sector. Also whether net or gross debt is used.]

2/ Gross financing need is defined as the primary deficit plus debt service plus the stock of short-term debt at the end of the last period

$3 /$ Revenues excluding grants.

4/ Debt service is defined as the sum of interest and amortization of medium and long-term debt.

$5 /$ Historical averages and standard deviations are generally derived over the past 10 years, subject to data availability 
Figure 1. Mozambique: Indicators of Public and Publicly Guaranteed External Debt under Alternatives Scenarios, 2009-2029 1/
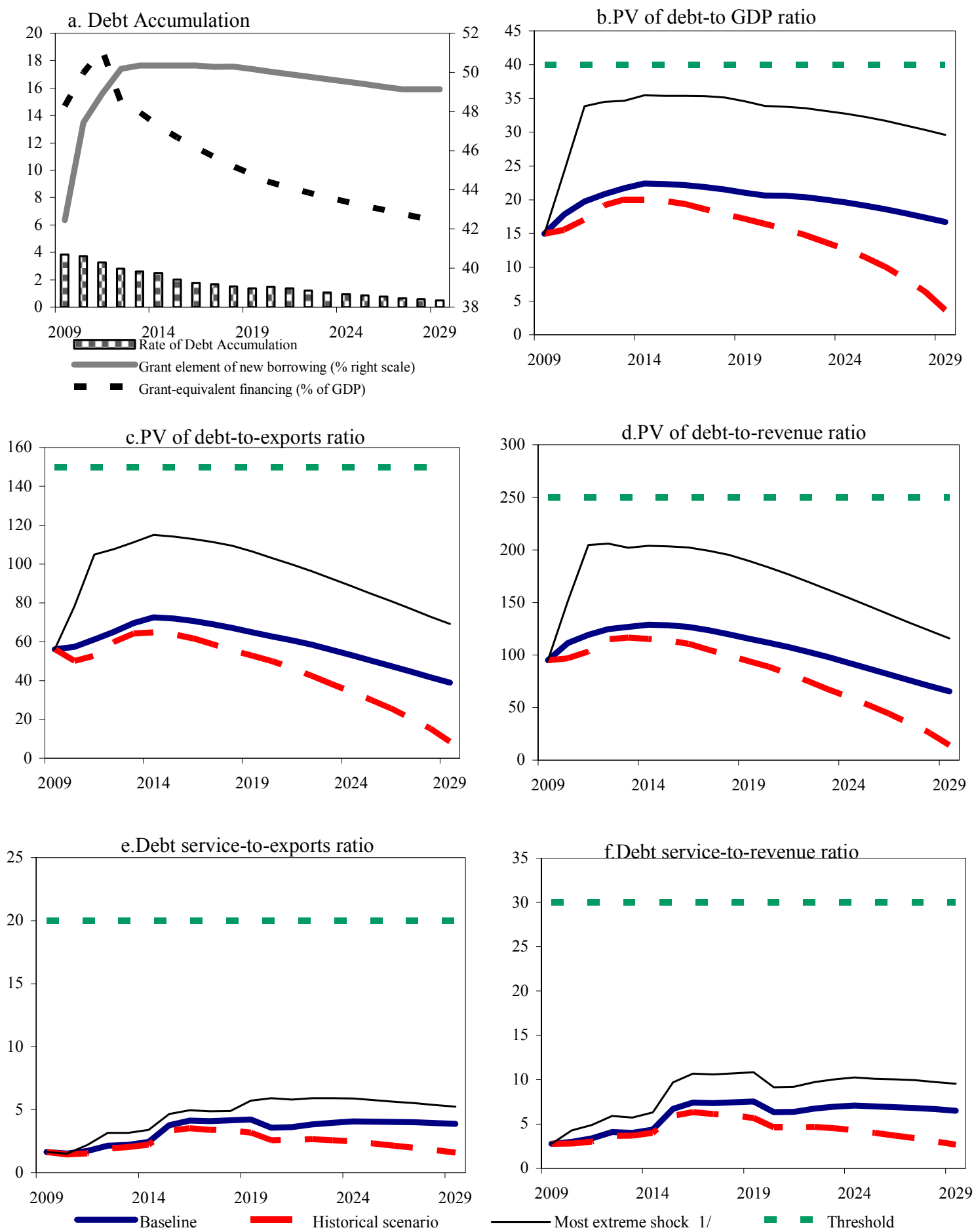

Sources: Country authorities; and staff estimates and projections.

1 / The most extreme stress test is the test that yields the highest ratio in 2019. In figure b. it corresponds to a Non-debt flows shock; in c. to a Non-debt flows shock; in d. to a Non-debt flows shock; in e. to a Non-debt flows shock and in figure f. to a One-time depreciation shock 


\section{AnNeX I. Relations With the FUnd}

(as of May 31, 2009)

I. Membership Status: Joined: September 24, 1984;

Article XIV

II. General Resources Account:

SDR Million

113.60

113.60

0.01

Reserve Position

Holdings Exchange Rate

III. SDR Department:

Holdings

IV. Outstanding Purchases and Loans:

PRGF Arrangements

$\begin{array}{rr}\text { SDR Million } & \text { \%Quota } \\ 113.60 & 100.00 \\ 113.60 & 100.00 \\ 0.01 & 0.01 \\ & \\ \text { SDR Million } & \text { N/A } \\ 0.07 & \text { \%Qllocation } \\ & 8.57\end{array}$

V. Latest Financial Arrangements:

\begin{tabular}{|c|c|c|c|c|}
\hline Type & $\begin{array}{c}\text { Date of } \\
\text { Arrangement }\end{array}$ & $\begin{array}{c}\text { Expiration } \\
\text { Date }\end{array}$ & $\begin{array}{c}\text { Amount Approved } \\
\text { (SDR Million) }\end{array}$ & $\begin{array}{c}\text { Amount Drawn } \\
\text { (SDR Million) }\end{array}$ \\
\hline PRGF & Jul 06, 2004 & Jul 05, $\overline{2007}$ & 11.36 & 11.36 \\
\hline PRGF & Jun 28, 1999 & Jun 28, 2003 & 87.20 & 78.80 \\
\hline PRGF & Jun 21, 1996 & Jun 27, 1999 & 75.60 & 75.60 \\
\hline
\end{tabular}

VI. Projected Payments to Fund ${ }^{1}$

(SDR Million; based on existing use of resources and present holdings of SDRs):

\begin{tabular}{|c|c|c|c|c|c|}
\hline & & & oming & & \\
\hline & 2009 & 2010 & 2011 & $\underline{2012}$ & 2013 \\
\hline Principal & & 0.16 & 0.97 & 1.46 & 1.95 \\
\hline Charges/Interest & 0.05 & 0.05 & 0.05 & 0.04 & 0.03 \\
\hline Total & 0.05 & 0.21 & 1.02 & 1.50 & 1.98 \\
\hline
\end{tabular}

1 When a member has overdue financial obligations outstanding for more than three months, the amount of such arrears will be shown in this section. 
VII. Implementation of HIPC Initiative:

I. Commitment of HIPC assistance

Decision point date

Assistance committed

by all creditors (US $\$$ Million) ${ }^{1}$

Of which: IMF assistance (US\$ million)

(SDR equivalent in millions)

\begin{tabular}{|c|c|}
\hline Original & Enhanced \\
\hline amework & Framewo \\
\hline Apr 1998 & Apr 20 \\
\hline $1,716.00$ & 306. \\
\hline 124.60 & \\
\hline 93.17 & \\
\hline Jun 199 & Sep \\
\hline
\end{tabular}

Completion point date

Sep 2001

II. Disbursement of IMF assistance (SDR Million)

Assistance disbursed to the member

93.17

13.73

106.90

Interim assistance

2.31

Completion point balance

93.17

11.42

104.58

Additional disbursement of interest income ${ }^{2}$

1.10

1.10

Total disbursements

93.17

14.83

108.00

${ }^{1}$ Assistance committed under the original framework is expressed in net present value (NPV) terms at the completion point, and assistance committed under the enhanced framework is expressed in NPV terms at the decision point. Hence these two amounts can not be added.

${ }^{2}$ Under the enhanced framework, an additional disbursement is made at the completion point corresponding to interest income earned on the amount committed at the decision point but not disbursed during the interim period.

VIII. Implementation of Multilateral Debt Relief Initiative (MDRI):

I. MDRI-eligible debt (SDR Million) ${ }^{1}$

106.56

Financed by: MDRI Trust

Remaining HIPC resources

II. Debt Relief by Facility (SDR Million)

Eligible Debt

$\begin{array}{lrrr}\frac{\text { Delivery Date }}{\text { January } 2006} & \frac{\text { GRA }}{\text { N/A }} & \frac{\text { TRGF }}{106.56} & \frac{\text { Total }}{106.56}\end{array}$

1 The MDRI provides 100 percent debt relief to eligible member countries that qualified for the assistance. Grant assistance from the MDRI Trust and HIPC resources provide debt relief to cover the full stock of debt owed to the Fund as of end-2004 that remains outstanding at the time the member qualifies for such debt relief. 


\section{Safeguards assessment}

At the request of the Bank of Mozambique, FIN conducted a voluntary safeguards assessment which was concluded in June 2008. This assessment updated the findings of an earlier assessment completed in August 2004. The 2008 assessment noted progress in implementing the recommendations of the earlier assessment. However weaknesses remain in the external and internal audit functions, financial reporting, and control systems.

\section{Exchange arrangements}

Mozambique has a floating exchange system. Commercial banks may buy and sell foreign exchange to individual customers on a fully negotiable basis. The Bank of Mozambique introduced a foreign exchange auction system in January 2005. Foreign exchange transactions are conducted mainly bilaterally and there is no pre-established frequency for auctions.

Mozambique still avails itself of the transitional arrangements under Article XIV of the Fund Articles of Agreement, but has eliminated all Article XIV restrictions. It maintains, however, restrictions on the making of payments and transfers for current international transactions subject to Fund approval under Article VIII, as evidenced by (i) the discretionary prior approval for remittances of family living expenses; (ii) the authorization for the purchase of foreign exchange in excess of US\$5,000 for certain transactions; (iii) the prohibition for the conversion of balances of nonresidents' domestic currency accounts into foreign currency or transfer abroad; (iv) the prohibition on advance payments for a service; and (v) the prohibition on advance payments for the import of goods. On June 18, 2007, the Fund granted approval for the retention of these five exchange restrictions until May 31, 2008. At the authorities' request, an Article VIII mission was conducted by LEG and PDR in March 2004. A new foreign exchange law was submitted to the Assembly in May 2007. Following enactment of the new law and issuance of related regulations, the authorities intend to accept their obligations under Article VIII sections 2, 3, and 4 of the Fund's Articles of Agreement.

\section{Article IV consultation}

In accordance with Decision No 12794-(02/76), as amended by Decision No 12854-(02/96), Mozambique is on a 24-month Article IV cycle due to the approval of a PSI in June 2007. The 2007 Article IV consultation was completed by the Executive Board on June 18, 2007 (Country Report No. 07/262).

In concluding the 2007 Article IV consultation, Executive Directors commended the Mozambican authorities on the impressive macroeconomic performance and overall satisfactory program performance since the last Article IV consultation. At the same time, they noted there are policy challenges related to sustaining Mozambique's growth takeoff and to achieving the MDGs. In this regard, they agreed that the strategy to consolidate macroeconomic stability in the context of a continued scaling-up of foreign aid and the acceleration of a second wave of reforms outlined in the authorities' poverty reduction plan for 2006-09 (PARPA II) would be essential to overcome such challenges. In particular, they looked forward to the timely implementation of reforms to 
(i) increasing tax revenues; (ii) strengthening public sector operations; (iii) reducing the costs of doing business; and (iv) improving the management of natural resources.

\section{Ex-post assessment of performance under Fund-supported programs}

An ex post assessment (EPA) of Mozambique's performance under Fund-Supported Programs since 1987 was undertaken in December 2003 under the guidelines on assessments of countries with a longer-term program engagement. The EPA was circulated to the Executive Board on November 21, 2003 (Country Report No. 04/53).

Directors commended the authorities for their pursuit of sound macroeconomic policies and wide ranging structural reforms over the past fifteen years which contributed to strong growth of the economy, and a steady decline in poverty rates. Noting that despite considerable progress Mozambique remains a very poor and vulnerable country, Directors urged the authorities to persevere in their efforts to consolidate macroeconomic stability and accelerate and deepen structural reforms with a view to sustaining economic growth, encouraging employment creation, and further reducing poverty.

\section{FSAP participation and ROSCs}

A Financial Sector Assessment Program (FSAP) for Mozambique was undertaken during the first quarter of 2003. The related Financial Sector Stability Assessment was circulated to the Executive Board on November 19, 2003 (Country Report No. 04/52). An update to the FSAP took place in February 2009, and the follow-up mission to discuss a timetable for implementing the FSAP recommendations took place in May 2009. A ROSC on fiscal transparency was issued on February 22, 2001. This ROSC was updated in the context of the 2002 Article IV consultation (Country Report No. 02/140) and the 2003 Article IV consultation (Country Report No. 04/50). The ROSC on fiscal transparency was updated in May 2008. A Report on the Observance of Standards and Codes (ROSC) data module was prepared in June 2002 and issued on March 5, 2003. This data module was updated in August 2005.

\section{Management's visit}

At the invitation of the authorities, Mr. Kato, Deputy Managing Director, visited Maputo, Mozambique in July 2005.The Managing Director visited Mozambique on August 2007 for a meeting with the African Consultative Group on Quotas, Voice, and Representation. 


\begin{tabular}{|c|c|c|c|c|}
\hline \multicolumn{5}{|c|}{$\begin{array}{l}\text { IMF Technical Assistance Provided to Mozambique } \\
\qquad(2006-09)\end{array}$} \\
\hline Departments & Timing & Form & Purpose & Counterparts \\
\hline Finance & February 2008 & Mission & Update of safeguard assessment & Bank of Mozambique \\
\hline \multirow[t]{11}{*}{ Fiscal Affairs } & May 2009 & Mission & Tax Administration Reform & Ministry of Finance \\
\hline & August 2008 & Mission & $\begin{array}{l}\text { Public Financial Management } \\
\text { Reforms }\end{array}$ & Ministry of Finance \\
\hline & April-May 2008 & Mission & Revenue Administration reform & Ministry of Finance \\
\hline & Oct-Nov 2007 & Mission & Tax Administration inspection & Ministry of Finance \\
\hline & September 2007 & Mission & $\begin{array}{l}\text { Public Private Partnerships and } \\
\text { Fiscal Risks }\end{array}$ & Ministry of Finance \\
\hline & August 2007 & Peripatetic expert & Tax Policy and Administration & Ministry of Finance \\
\hline & August 2007 & Mission & $\begin{array}{l}\text { Fiscal ROSC update and Public } \\
\text { Financial Management inspection }\end{array}$ & Ministry of Finance \\
\hline & April-May 2007 & Mission & Tax Administration inspection & Ministry of Finance \\
\hline & April-May 2007 & Mission & Petroleum Fiscal Regime & $\begin{array}{l}\text { Ministry of Finance } \\
\text { Ministry of Mineral } \\
\text { Resources }\end{array}$ \\
\hline & December 2006 & Mission & Tax Administration & Ministry of Finance \\
\hline & March 2006 & Mission & Tax Policy review & Ministry of Finance \\
\hline \multirow{10}{*}{$\begin{array}{l}\text { Monetary and } \\
\text { Capital Markets }\end{array}$} & May 2009 & Mission & 2009 FSAP follow-up mission & Bank of Mozambique \\
\hline & February 2009 & Mission & $\begin{array}{l}\text { TA on Central Banking and } \\
\text { Monetary Policy Formulation }\end{array}$ & Bank of Mozambique \\
\hline & May 2008 & Mission & $\begin{array}{l}\text { Strengthening Monetary Policy } \\
\text { Formulation and Implementation }\end{array}$ & Bank of Mozambique \\
\hline & December 2007 & Mission & $\begin{array}{l}\text { Implementation of IFRS for Central } \\
\text { Bank accounts. }\end{array}$ & Bank of Mozambique \\
\hline & August 2007 & Mission & $\begin{array}{l}\text { Post-FSAP TA assessment } \\
\text { banking supervision, monetary } \\
\text { Policy and operations }\end{array}$ & Bank of Mozambique \\
\hline & April 2007 & Short-term consultant & Banking supervision & Bank of Mozambique \\
\hline & October 2006 & Mission & $\begin{array}{l}\text { Post- FSAP TA assessment } \\
\text { banking supervision, monetary } \\
\text { Policy and operations }\end{array}$ & Bank of Mozambique \\
\hline & Feb-March 2006 & Mission & AML/CFT legislation & Bank of Mozambique \\
\hline & Jan-Feb 2006 & Short-term consultant & Monetary policy framework & Bank of Mozambique \\
\hline & Jan-Feb 2006 & Short-term consultant & Monetary operations & Bank of Mozambique \\
\hline
\end{tabular}




\begin{tabular}{|l|l|l|l|l|}
\hline \multicolumn{2}{|c|}{ IMF Technical Assistance Provided to Mozambique } \\
(2006-09)
\end{tabular}

Resident Representative: Mr. Felix Fischer has been the IMF's resident representative to Mozambique since March 14, 2006. 
Annex II. World Bank-IMF Joint Management ACtion Plan

\begin{tabular}{|c|c|c|c|}
\hline \multicolumn{4}{|c|}{ Mozambique: World Bank-IMF Joint Management Action Plan } \\
\hline Title & Products & Timing of Missions & Expected delivery date \\
\hline \multicolumn{4}{|c|}{$\begin{array}{l}\text { A. Mutual information on relevant work program in } \\
\text { next } 12 \text { months }\end{array}$} \\
\hline \multirow[t]{4}{*}{ Bank work program } & CEM on export competitiveness and regional integration & Feb-May 2008 & June 2009 \\
\hline & $\begin{array}{l}\text { PRSC6 supporting government reforms program in PFM, PSR and economic } \\
\text { development reforms }\end{array}$ & & November 2009 \\
\hline & Updated poverty analysis using the $2008 / 09$ household survey & & February 2010 \\
\hline & FSTAP project support to financial sector reforms & & ongoing \\
\hline \multirow[t]{4}{*}{ Fund work program } & Fourth Review of the PSI & May-09 & Jul-09 \\
\hline & Follow up mission on Tax Administration & May-09 & Jul-09 \\
\hline & Mission on PFM reforms & Aug-08 & Sep-08 \\
\hline & Follow up mission on the February 2009 FSAP update & May-09 & Jul-09 \\
\hline \multicolumn{4}{|c|}{$\begin{array}{l}\text { B. Requests for work program inputs with summary } \\
\text { justification }\end{array}$} \\
\hline \multirow[t]{2}{*}{ Bank request to the Fund } & Updated Macro Framework data and PSI review Staff Report & PSI reviews & October 2009 - April 2010 \\
\hline & $\begin{array}{l}\text { Board Chairman Summing up from PSI review discussion (or Assessment } \\
\text { letter) not older than six-months }\end{array}$ & & September 2009 \\
\hline Fund request to the Bank & $\begin{array}{l}\text { Updates on advancing structural reforms: procurement, decentralization, } \\
\text { business environment, financial sector }\end{array}$ & & September and October 2009 \\
\hline \multicolumn{4}{|c|}{ C. Agreements on joint products and missions } \\
\hline \multirow[t]{7}{*}{ Joint products } & DSA & End-2009 & Early 2010 \\
\hline & FSAP & February 2009 & May 2009 (delivered) \\
\hline & Support to EITI membership, and scoping report on EITI++ & & October 2008 (delivered) \\
\hline & $\begin{array}{l}\text { PFM: update work plan for 2009-11 in light of findings from PEFA and } \\
\text { ROSC }\end{array}$ & & October 2010 \\
\hline & PFM: continue to monitor consolidation and expansion of e-SISTAFE. & ongoing & ongoing \\
\hline & Policy advice on Public Sector Salary Reforms (incl. pensions) & ongoing & ongoing \\
\hline & Review of tax policy & tbd & tbd \\
\hline
\end{tabular}




\section{ANNEX III: STATISTICAL ISSUES}

1. Data provision has some shortcomings, but is broadly adequate for surveillance. Despite the increase in budget resources allocated to the compilation of official statistics, continued high reliance on external funding raises concerns about the sustainability of the programs of the National Institute of Statistics (INE). The authorities are making efforts to improve the quality and timeliness of economic and financial data through participation in the Fund's General Data Dissemination System (GDDS) and in the external sector module of the GDDS Project for Anglophone African Countries (funded by the U.K. Department for International Development (DFID)). GDDS metadata have been posted on the Fund's Dissemination Standards Bulletin Board (DSBB) since November 2003.

2. In May 2005, a STA mission updated the data module of the Report on the Observance of Standards and Codes (ROSC) prepared in June 2002. The mission noted that significant effort has been made to address previously identified shortcomings. The improvements in the institutional environment and the increased allocation of resources for the compilation of national accounts, balance of payments, and more recently, government finance statistics should help to address weaknesses in the prerequisites for the quality of the statistics. The methodological soundness, accuracy, and reliability of macroeconomic statistics are starting to show improvements as a result of these actions.

\section{National accounts}

3. The national accounts are prepared by the INE in accordance with the 1993 System of National Accounts (1993 SNA). In August 2007, the INE released for the first time quarterly GDP estimates at constant prices covering 2000-07, along with a revised annual GDP series for the period 1991-2006, having 2003 as the new base year. A (March-April 2008) STA mission advised the authorities on further improvements needed to advance the quarterly national accounts compilation at current prices, that could be released starting in mid-2009. The INE has launched a new household income and expenditure survey and new economic censuses leading to a new business registry. Furthermore, it is compiling more comprehensive and timely foreign trade data based on improved classification systems.

\section{Prices and labor market}

4. A revamped consumer price index for Maputo based on weights derived from the 2002-03 household survey was released in February 2006. A national index obtained by integrating the indices for Maputo, Beira, and Nampula was released in April 2006. A STA mission in June 2006 found important advances regarding the use of the COICOP classification and the strengthening of human resources in the compilation of the CPI. Several issues remain outstanding, however, such as price collection procedures not fully consolidated at the provincial level, imputation methods, and possible inconsistencies in the overlapping period between the new and old series. 
5. There are insufficient sectoral labor market and employment data, and where available, they have limited coverage. A one-year labor market survey of the entire country, undertaken by INE in collaboration with the Labor Ministry, was launched in October 2004.

\section{Monetary statistics}

6. A STA mission in September 2004 prepared a work plan for the implementation of the Monetary and Financial Statistics Manual (MFSM) and the development of an integrated monetary database (IMD) to meet the needs of the Bank of Mozambique (BM), AFR, and STA. The mission recommended improving the classification and valuation of some financial instruments, estimating the full instrument and sectoral breakdown based on available information, and expanding the coverage of the survey on other depository corporations to include credit cooperatives.

7. A follow-up monetary and financial statistics mission in November 2007 assisted the authorities in finalizing the IMD, which allow the derivation of accurate and timely monthly monetary statistics, while reducing BM's reporting burden. Mozambique now reports monthly monetary data to STA using the standardized reporting forms (SRFs). As a result, five-year historical enhanced data are being published in the International Financial Statistics (IFS) Supplement.

\section{External sector statistics}

8. With assistance from STA, provided in the context of the GDDS regional project, the $\mathrm{BM}$ has made significant progress toward compiling and disseminating balance of payments (BOP) and international investment position (IIP) statistics that are fully aligned with the Balance of Payments Manual, fifth edition (BPM5).

9. The BM has an adequate institutional framework for the compilation of BOP and IIP statistics, and has implemented many of the recommendations made by the four technical assistance missions conducted since mid-2003. However, in order for the work on institutional capacity building to be consolidated, the Foreign Exchange Law has to be approved (incorporating the definition of residency in line with the BPM5 methodology), training on BOP statistics has to be sustained, and the project to computerize the balance of payments compilation system has to be completed. Although, major improvements were achieved in the basic data sources since the beginning of the project, remaining concerns include (i) the coverage and quality of the data obtained in the enterprise surveys; (ii) the quality of external trade data, especially with regard to price and volume indices; (iii) the coverage, time of recording, and classification of the data on foreign investment and private loans; (iv) completeness of data for the International Reserves Template and their reconciliation with the reserve component of the balance of payments; and (v) compiling the IIP using the sources that are used for the balance of payments compilation. To improve the collection, timeliness, and quality of merchandise trade data, a panel on foreign trade 
statistics was established, comprising representatives from the Directorate General of Customs, National Statistics Institute, and BM.

10. In 2007, the BM started to compile and report to the STA comprehensive IIP data. Previously, only partial IIP data had been reported and published in the Balance of Payments Statistics Yearbook.

\section{Government finance statistics}

11. A July 2005 STA mission found serious weaknesses in fiscal statistics. The budget accounting system, broadly aligned to the GFSM 1986 analytical framework, had shortcomings relating to: definitions and coverage of institutional units included in the general government sector; budgetary classifications not fully meeting GFS needs; and the omission of certain revenues and expenditures. Mozambique does not report fiscal data for publication in the IFS or the Government Finance Statistic Yearbook (GFSY). 
Mozambique: Table of Common Indicators Required for Surveillance (As of December 4, 2008)

\begin{tabular}{|c|c|c|c|c|c|c|c|}
\hline & \multirow{2}{*}{$\begin{array}{l}\text { Date of latest } \\
\text { observation }\end{array}$} & \multirow[t]{2}{*}{ Date received } & \multirow{2}{*}{$\begin{array}{c}\text { Frequency of } \\
\text { Data }^{7}\end{array}$} & \multirow{2}{*}{$\begin{array}{l}\text { Frequency of } \\
\text { Reporting }^{7}\end{array}$} & \multirow{2}{*}{$\begin{array}{l}\text { Frequency of } \\
\text { publication }^{7}\end{array}$} & \multicolumn{2}{|c|}{ Memo Items: } \\
\hline & & & & & & $\begin{array}{c}\text { Data Quality - } \\
\text { Methodological soundness }^{8}\end{array}$ & $\begin{array}{c}\text { Data Quality Accuracy } \\
\text { and reliability }{ }^{9}\end{array}$ \\
\hline Exchange Rates & Sep. 2008 & Oct. 2008 & $\mathrm{D}$ & W & $\mathrm{D}$ & & \\
\hline \multicolumn{8}{|l|}{$\begin{array}{l}\text { International Reserve Assets and Reserve Liabilities of the } \\
\text { Monetary Authorities }{ }^{1}\end{array}$} \\
\hline Reserve/Base Money & Mar. 2009 & May. 2009 & $\mathrm{M}$ & $\mathrm{M}$ & $\mathrm{M}$ & \multirow[t]{4}{*}{ LO, O, LO, O } & \multirow[t]{4}{*}{ LO, LO, O, O, LO } \\
\hline Broad Money & Mar. 2009 & May. 2009 & $\mathrm{M}$ & $\mathrm{M}$ & $\mathrm{M}$ & & \\
\hline Central Bank Balance Sheet & Mar. 2009 & May. 2009 & $\mathrm{M}$ & $\mathrm{M}$ & $\mathrm{M}$ & & \\
\hline Consolidated Balance Sheet of the Banking System & Mar. 2009 & May. 2009 & & & & & \\
\hline Interest Rates $^{2}$ & Mar. 2009 & May. 2009 & $\mathrm{M}$ & $\mathrm{M}$ & $\mathrm{M}$ & & \\
\hline Consumer Price Index & Mar. 2009 & May. 2009 & $\mathrm{M}$ & $\mathrm{M}$ & $\mathrm{M}$ & $\mathrm{O}, \mathrm{LO}, \mathrm{LNO}, \mathrm{O}$ & LNO, LO, LO, O, O \\
\hline \multicolumn{6}{|l|}{$\begin{array}{l}\text { Revenue, Expenditure, Balance and Composition of Financing }{ }^{3} \\
- \text { General Government }^{4}\end{array}$} & \multirow[t]{2}{*}{ LO, LNO, LO, O } & \multirow[t]{2}{*}{ LO, O, LO, O, LNO } \\
\hline $\begin{array}{l}\text { Revenue, Expenditure, Balance and Composition of } \\
\text { Financing }{ }^{3}-\text { Central Government }\end{array}$ & Mar. 2009 & May. 2009 & Q & $Q$ & Q & & \\
\hline $\begin{array}{l}\text { Stocks of Central Government and Central Government- } \\
\text { Guaranteed Debt }^{5}\end{array}$ & Mar. 2009 & May. 2009 & $\mathrm{Q}$ & $\mathrm{Q}$ & $\mathrm{Q}$ & & \\
\hline External Current Account Balance & Dec. 2007 & Mar. 2008 & Q & I & 1 & \multirow[t]{2}{*}{ LO, LNO, LO, O } & \multirow[t]{2}{*}{$\begin{array}{l}\text { LO, LNO, LO, LO, } \\
\text { LNO }\end{array}$} \\
\hline Exports and Imports of Goods and Services & Dec. 2007 & Mar. 2008 & & & & & \\
\hline GDP/GNP & 2008 & Feb. 2009 & $\bar{A}$ & $\mathrm{I}$ & $\mathrm{I}$ & O, LO, O, LO & LNO, LO, LO, O, LO \\
\hline Gross External Debt & Dec. 2008 & Mar. 2009 & $\mathrm{~A}$ & $\mathrm{I}$ & $\mathrm{I}$ & & \\
\hline International Investment Position ${ }^{6}$ & 2007 & May 2009 & $\mathrm{~A}$ & A & A & & \\
\hline
\end{tabular}

\footnotetext{
${ }^{1}$ Includes reserve assets pledged or otherwise encumbered as well as net derivative positions.

${ }^{2}$ Both market-based and officially determined, including discount rates, money market rates, rates on treasury bills, notes and bonds.

${ }^{3}$ Foreign, domestic bank, and domestic nonbank financing.

${ }^{4}$ The general government consists of the central government (budgetary funds, extrabudgetary funds, and social security funds) and state and local governments.

${ }^{5}$ Including currency and maturity composition.

${ }^{6}$ Includes external gross financial asset and liability positions vis-à-vis nonresidents.

7 Includes external gross financial asset and liability positions vis-à-vis nonresidents.

Daily (D), Weekly (W), Monthly (M), Quarterly (Q), Annually (A), and Not Available (NA)

Reflects the assessment provided in the data ROSC or the Substantive Update for the dataset corresponding to the variable in each row. The assessment indicates whether international standards concerning concepts and definitions, scope, classification/sectorization, and basis for recording are fully observed (O); largely observed (LO); largely not observed (LNO); not observed (NO); and not available (NA)
} 
Public Information Notice (PIN) No. 09/85

FOR IMMEDIATE RELEASE

July 16, 2009

\section{IMF Executive Board Concludes 2009 Article IV Consultation with Mozambique}

On June 30, 2009, the Executive Board of the International Monetary Fund (IMF) concluded the Article IV consultation with Mozambique. ${ }^{1}$

\section{Background}

Mozambique has continued its record of strong macroeconomic performance since the mid1990s and its performance compares well with other countries in the region. Output growth in Mozambique over the past decade averaged almost 8 percent. Growth has been sustained by investment financed by large aid and private capital inflows, mainly into the natural resource sector. It has also been sustained by a strong trade sector-merchandise exports' share of GDP more than tripled to nearly 30 percent of GDP, eliminating a trade deficit of about 30 percent of GDP.

However, even before the current global crisis, growth had been weakening, with growth projections about 1 percentage point lower than in the past decade. In recent years, these flows contributed to an appreciation of currency in real effective terms. Growth has also been unevenly distributed and a recent survey suggests that poverty may have increased in recent years, particularly in rural areas.

Against a backdrop of a highly uncertain global economic environment, Mozambique's macroeconomic prospects remain positive, but have weakened. Mozambique's growth in 2009 could fall several percentage points below trend. In view of WEO projections for a slow global recovery, growth is projected to strengthen in 2010 , but will probably remain below 6 percent until 2011. Similarly, the external overall balance is projected to turn negative in both 2009; turning positive again in 2011 and stabilizing external reserves around 3.4 months of imports.

The scope for supportive macroeconomic policies to offset the impact of the global economic slowdown is curtailed by the large projected decline in external reserves. Most of the deterioration in the external overall balance is the direct result of the external shock; the

\footnotetext{
${ }^{1}$ Under Article IV of the IMF's Articles of Agreement, the IMF holds bilateral discussions with members, usually every year. A staff team visits the country, collects economic and financial information, and discusses with officials the country's economic developments and policies. On return to headquarters, the staff prepares a report, which forms the basis for discussion by the Executive Board. At the conclusion of the discussion, the Managing Director, as Chairman of the Board, summarizes the views of Executive Directors, and this summary is transmitted to the country's authorities.
} 
fiscal expansion is estimated to account for only US $\$ 42$ million of the reserve loss in 2009 through higher import demand.

Mozambique is eligible for a one-year arrangement under the ESF with access of 100 percent of quota (SDR 113.6 million) to be made available in three drawings following completion of the fourth, fifth and sixth reviews under the PSI. Mozambique faces balance of payments difficulties caused primarily by a sudden and exogenous shock. Moreover, the shock is expected to be temporary, as the overall balance is projected to revert to a surplus in 2011. Access to the ESF, would provide timely coverage of about two thirds of the direct balance of payments impact of the external shock. Moreover, the use of Fund resources should catalyze support from donors which are considering stepping up their assistance, most notably the World Bank.

\section{Executive Board Assessment}

Executive Directors welcomed Mozambique's continued strong macroeconomic performance in 2008, despite a challenging external environment. Directors commended the authorities' flexible policy response to higher fuel and food import prices, which helped to mitigate the impact of these shocks. Looking forward, they noted that Mozambique remains vulnerable to the global economic slowdown and encouraged the authorities to monitor international and domestic developments closely.

Directors agreed that Mozambique's strong track record of prudent macroeconomic policy implementation has provided room to ease fiscal and monetary policy in the nearterm, while remaining within its prudent medium-term strategy. In this context, they welcomed the authorities' commitment to expenditure restraint in the approach to elections, and encouraged them to protect the most vulnerable from the consequences of the economic crisis.

In the medium-term, Directors underscored the importance of further strengthening macroeconomic stability, and supported efforts to enhance domestic revenue mobilization and to improve public financial management. Recourse to domestic budget financing should be avoided so as to provide room for credit to the private sector. Monetary policy should aim to keep inflation low. Directors welcomed steps to strengthen monetary policy formulation and implementation. They encouraged the authorities to intensify their efforts to diversify the sources of growth and to accelerate structural reforms to boost Mozambique's competitiveness, promote investment, and improve financial intermediation.

Directors took note of the staff's assessment that Mozambique's currency is overvalued in real effective terms, while recognizing the methodological constraints affecting this evaluation, particularly in the context of an economy undergoing significant structural changes, and emphasized the potentially adverse impact this could have on competitiveness and economic diversification. They encouraged the authorities to fulfill their commitment to exchange rate flexibility.

Directors commended the authorities on Mozambique's continued advances on its structural reform agenda. They welcomed the progress in strengthening fiscal and monetary operations and called for urgent and rigorous reforms to strengthen the private sector and reduce the costs of doing business. Directors were encouraged by the authorities' efforts to 
improve governance including the development of anti-corruption policies and by Mozambique's formal acceptance by the Extractive Industries' Transparency Initiative Board.

Directors welcomed the FSAP Update's finding that Mozambique has made significant progress towards developing and strengthening the financial sector and improving banking operations. They also welcomed the authorities' commitment to move ahead quickly to assess and finalize an action plan to implement the FSAP Update's recommendations, including strengthening risk monitoring, home-host cooperation on banking supervision, and crisis management arrangements.

Directors welcomed the authorities' intention to accept the obligations under Article VIII.

Public Information Notices (PINs) form part of the IMF's efforts to promote transparency of the IMF's views and analysis of economic developments and policies. With the consent of the country (or countries) concerned, PINs are issued after Executive Board discussions of Article IV consultations with member countries, of its surveillance of developments at the regional level, of post-program monitoring, and of ex post assessments of member countries with longer-term program engagements. PINs are also issued after Executive Board discussions of general policy matters, unless otherwise decided by the Executive Board in a particular case. 
Mozambique: Selected Economic and Financial Indicators, 2007-10

\begin{tabular}{|c|c|c|c|c|}
\hline & 2007 & 2008 & 2009 & 2010 \\
\hline & Act. & Est. & Proj. & Proj. \\
\hline & \multicolumn{3}{|c|}{$\begin{array}{l}\text { (Annual percentage change, unless otherwise } \\
\text { indicated) }\end{array}$} & \\
\hline \multicolumn{5}{|l|}{ National income and prices } \\
\hline Nominal GDP (billions of MT) & 208 & 239 & 261 & 288 \\
\hline Nominal GDP growth & 15.4 & 15.0 & 9.1 & 10.2 \\
\hline Real GDP growth & 7.4 & 6.8 & 4.3 & 5.0 \\
\hline GDP per capita (U.S. dollars) & 398 & 477 & 440 & 445 \\
\hline Consumer price index (annual average) & 8.2 & 10.3 & 6.1 & 5.6 \\
\hline Consumer price index (end of period) & 10.3 & 6.2 & 5.8 & 5.7 \\
\hline \multicolumn{5}{|l|}{ External sector } \\
\hline Merchandise exports & 1.3 & 10.0 & -29.3 & 25.7 \\
\hline Merchandise exports, excluding megaprojects & -17.9 & 41.1 & -14.7 & 6.2 \\
\hline Merchandise imports & 4.8 & 23.0 & -3.5 & -1.8 \\
\hline Merchandise imports, excluding megaprojects & 4.7 & 26.9 & -11.6 & 14.1 \\
\hline Terms of trade & 4.2 & -15.5 & -21.2 & 6.4 \\
\hline Nominal effective exchange rate (end of period) ${ }^{1}$ & 2.2 & 22.5 & $\ldots$ & $\ldots$ \\
\hline Real effective exchange rate (end of period) ${ }^{1}$ & 6.5 & 24.3 & $\ldots$ & $\ldots$ \\
\hline & \multicolumn{3}{|c|}{$\begin{array}{l}\text { (Annual changes in percent of beginning-of-period } \\
\text { stock of money-M3, unless otherwise indicated) }\end{array}$} & \\
\hline \multicolumn{5}{|l|}{ Money and credit ${ }^{2}$} \\
\hline Net foreign assets & 11.8 & 4.0 & -0.1 & $\ldots$ \\
\hline Net domestic assets & 13.4 & 16.3 & 15.1 & $\ldots$ \\
\hline Of which: net credit to the government ${ }^{3}$ & 3.0 & -2.5 & 5.8 & $\ldots$ \\
\hline credit to the economy & 6.5 & 21.1 & 15.6 & $\ldots$ \\
\hline Broad money-M3 (12-month percent change) & 25.2 & 20.3 & 15.0 & $\ldots$ \\
\hline M2 (12-month percent change) & 23.0 & 26.0 & 15.8 & $\ldots$ \\
\hline Velocity (GDP/M2) & 4.9 & 4.4 & 4.2 & $\ldots$ \\
\hline \multirow[t]{2}{*}{ Interest rate for 90 -day treasury bills (percent; end of period) } & 14.8 & 14.0 & $\ldots$ & $\ldots$ \\
\hline & \multicolumn{4}{|c|}{ (Percent of GDP) } \\
\hline \multicolumn{5}{|l|}{ Investment and saving } \\
\hline Gross domestic investment & 17.9 & 18.5 & 21.9 & 22.7 \\
\hline Government & 11.7 & 11.6 & 15.6 & 16.8 \\
\hline Other sectors & 6.2 & 6.9 & 6.3 & 5.9 \\
\hline Gross domestic savings (excluding grants) & -1.5 & -1.9 & -1.1 & -0.5 \\
\hline Government & 0.6 & 0.3 & -1.0 & -0.6 \\
\hline Other sectors & -2.1 & -2.2 & -0.1 & 0.1 \\
\hline Current account, before grants & -19.4 & -20.4 & -22.9 & -23.2 \\
\hline \multicolumn{5}{|l|}{ Government budget } \\
\hline Total revenue & 15.9 & 16.0 & 15.7 & 16.0 \\
\hline Total expenditure and net lending & 28.1 & 27.9 & 33.1 & 34.4 \\
\hline Overall balance, before grants & -13.1 & -11.6 & -17.4 & -18.4 \\
\hline Total grants & 9.3 & 9.5 & 11.8 & 14.0 \\
\hline Overall balance, after grants & -3.8 & -2.2 & -5.6 & -4.3 \\
\hline Domestic primary balance & -3.4 & -3.1 & -5.6 & -5.2 \\
\hline External financing (incl. debt relief) ${ }^{3}$ & 3.6 & 4.0 & 3.4 & 3.5 \\
\hline \multirow[t]{2}{*}{ Net domestic financing ${ }^{4}$} & 0.2 & -1.9 & 1.8 & 0.8 \\
\hline & \multicolumn{4}{|c|}{ (Millions of U.S. dollars, unless otherwise indicated) } \\
\hline External current account, before grants & $-1,576$ & $-2,021$ & $-2,135$ & $-2,229$ \\
\hline Overall balance of payments & 136 & 159 & -223 & -146 \\
\hline Net international reserves (end of period) & 1,508 & 1,644 & 1,421 & 1,275 \\
\hline Gross international reserves (end of period) & 1,520 & 1,660 & 1,440 & 1,294 \\
\hline In months of imports of goods and nonfactor services & 4.7 & 4.4 & 3.9 & 3.6 \\
\hline
\end{tabular}

Sources: Mozambican authorities; and IMF staff estimates and projections.

${ }^{1}$ Minus sign indicates depreciation.

${ }^{2}$ Data from 2008 onwards based on data following technical revisions to monetary survey finalized in January 2008.

${ }^{3}$ Includes movement in the government account set abroad with the proceed of the Moatize coal mine concession.

${ }^{4}$ Includes World Bank disbursement of 2009 budget support in 2008. 
June 30, 2009

\section{IMF Executive Board Approves US\$176 Million Exogenous Shocks Facility for Mozambique and Completes Fourth Review Under the Policy Support Instrument}

The Executive Board of the International Monetary Fund (IMF) today approved a 12-month, SDR 113.6 million (about US\$176 million) for Mozambique under the IMF's Exogenous Shocks Facility (ESF) to cushion the country from the effects of the global economic downturn. The approval enables Mozambique to draw immediately an amount equivalent to SDR 85.2 million (about US\$132 million). The IMF financing will help Mozambique offset the deterioration of its balance of payments, which has been undermined by the global economic downturn. The ESF is designed to provide policy support and financial assistance on concessional terms to eligible lowincome countries facing temporary exogenous shocks.

The IMF Executive Board also completed the fourth review of Mozambique's economic performance under the three-year Policy Support Instrument (PSI). The PSI for Mozambique was approved on June 18, 2007 (See Press Release No 07/135) to support the nation's economic reform efforts. It is aimed at consolidating macroeconomic stability and at achieving sustained economic growth and poverty reduction through the pursuit of prudent macroeconomic policies as well as promoting structural reforms. The strategy to achieve this goal remains set in the Mozambican authorities' national poverty reduction strategy, Plano de Acção para Redução da Pobreza Absoluta (PARPA II).

Following the Executive Board's discussion of Mozambique, Mr. Takatoshi Kato, Deputy Managing Director and Acting Chair, stated:

"Mozambique continued with a strong macroeconomic performance in 2008. A flexible policy response to higher fuel and food import prices helped to mitigate the impact of internal and external shocks in a challenging external environment. As Mozambique remains vulnerable to the global economic slowdown, international and domestic developments will need to be closely monitored.

“Mozambique's strong track record of prudent macroeconomic policy implementation has provided room for an easing of fiscal and monetary policy in the near-term while remaining within its prudent medium-term strategy. In this context, the authorities' commitment to expenditure restraint in the approach to elections is welcome. 
"In the medium-term, steps to enhance domestic revenue mobilization and to improve public financial management will contribute importantly to further strengthening macroeconomic stability. Avoiding recourse to domestic financing of the budget will provide room for credit to the private sector. Strengthened monetary policy formulation and implementation will help to maintain low inflation. Intensified efforts to diversify the sources of growth and to accelerate structural reforms will boost Mozambique's competitiveness, promote investment, and improve financial intermediation.

"Closer attention to a broader basket of currencies that is more reflective of Mozambique's trading patterns, could help to redress the overvaluation of the currency in real effective terms, which could have adverse impact on Mozambique's competitiveness and economic diversification.

"Mozambique has continued to advance on its structural reform agenda. Fiscal and monetary operations have been strengthened and an enhanced business environment for both domestic and foreign investment is targeted. Mozambique candidacy for adherence to the Extractive Industries' Transparency Initiative is welcome.

"Mozambique has made significant progress towards developing and strengthening the financial sector and improving banking operations. The authorities have committed to move ahead quickly to assess and finalize an action plan to further strengthen the financial sector, including risk monitoring, home-host cooperation on banking supervision, and crisis management arrangements," added Mr. Kato. 


\section{Statement by Samuel Itam, Executive Director for Republic of Mozambique June 30, 2009}

\section{Introduction}

1. My Mozambican authorities appreciate the continued support by the Fund and the constructive policy dialogue and advice by staff. They are committed to implementing reforms and to macroeconomic stability, as is evidenced by the satisfactory performance under the PSI arrangement since its inception. However, Mozambique is vulnerable to the global economic and financial crisis, and mitigating measures are necessary to meet the challenges and ensure that economic growth and macroeconomic stability are sustained. In this context, my Mozambican authorities are requesting a twelve-month arrangement under the high access component of the ESF.

2. The overall implementation of the PSI arrangement in the second half of 2008 was satisfactory. All quantitative assessment criteria (ACs) to end-December 2008 were met. Implementation of the two structural benchmarks for end-March 2009-the approval of a PFM vision paper and the issuance of a decree streamlining the business inspection process - were delayed and both are now expected to be implemented by end-July 2009. In this regard, the authorities request Directors' support for the completion of the fourth review of the PSI.

\section{Recent economic developments}

3. Macroeconomic performance during 2008 was good, despite pressures from the high fuel and food prices in mid-2008 together with the onset of the global economic crisis in late 2008. The economy is estimated to have expanded by 6.8 percent in 2008, with a particularly good performance in agriculture, construction, transport and communications and financial services. Inflation fell faster, from a peak of 13 percent in February 2008 to 6.2 percent in December 2008 and 3 percent in May 2009, mainly because of the sharp drop in import prices. The current account balance in 2008 was adversely affected by higher imports because of increases in international prices. However, because of high private capital inflows, an increase in gross international reserves was recorded for the year. The real effective exchange rate appreciated, but part of this appreciation has been reversed in the first months of 2009.

4. The Government's fiscal policy was revised in July 2008 to incorporate measures to mitigate the social impact of higher fuel and food prices. Domestic financing of the budget for the year was 0.8 percent of GDP lower than envisaged because of better than envisaged domestic revenues, from personal income tax, and lower than budgeted recurrent spending on goods and services. However, the late disbursements of funds for externally financed investment projects accounted for the overall low expenditure execution rate that was only 88 percent of the program. The multiplicity of accounting procedures, as well as the integration of projects into the budget, also influenced the rate of budget execution. In this regard, it 
should be noted that expenditure in PARPA's priority sectors accounts for almost two thirds of the total approved budget.

5. Monetary policy in $\mathbf{2 0 0 8}$ aimed at price stability. The target for reserve money was revised to accommodate the projected increase in inflation due to higher import prices. The Bank of Mozambique (BM) was able to contain reserve money growth in part due to strong fiscal performance. The BM and commercial banks have improved their management of the seasonal surge in currency demand over the festive season. Net international reserves were in line with revised projections and the program target was met.

6. Structural reforms to strengthen the management and transparency of mineral resources, hence maximizing the benefits to Mozambique, are on track. The authorities' request to become a candidate for adherence to the EITI was approved last May.

7. The strategy for the improvement of the business environment was approved by Cabinet, after which the following reforms were implemented: (i) elimination of the minimum capital and bank deposit requirement for the creation of companies;

(ii) introduction of the simplified licensing regime; and (iii) approval of the new Code of Tax Benefits. In the meantime, the authorities are committed to persevere with the improvement of the business environment, with a view to attract investments for the productive sectors, along with the associated creation of employment. These structural reforms are focused on:

(i) the streamlining of business inspections, particularly in the areas of labor and finance,

(ii) the contracting of foreign workers, taking into account the relevant regulation; and

(iii) the costs of doing international business.

\section{Outlook and policy response for 2009/10}

8. My authorities' policy thrust in $2009 / 10$ is to carefully monitor the impact of the global economic crisis on Mozambique's macroeconomic prospects and to implement appropriate policies to mitigate its effects. Against the backdrop of a highly uncertain global outlook, economic prospects for 2009 have deteriorated, and GDP growth is now projected to be far below the trend of the last few years. The external shocks will have adverse impacts on the balance of payments, with the overall position projected to deteriorate by nearly US $\$ 400$ million in 2009, as the trade deficit widens, foreign direct investment contracts, and foreign borrowing by the private sector is curtailed. To this end, the authorities have intensified their efforts to mobilize external financing.

9. On the fiscal side, revenues in 2009 are projected to be lower by 1.2 percent points of GDP compared with what was envisaged in the program (though by only -0.3 percent of GDP compared with the outturn for 2008). The authorities will maintain the domestic expenditure envelope in nominal terms broadly as previously envisaged and in line with budgeted levels. Therefore, relative to GDP, total spending will rise by about 5.2 percent of GDP compared with the outturn for 2008 (but will decline by -1.3 percent of GDP with respect to the envisaged program for 2009). To fill the financing gap, the authorities intend to increase net domestic financing to about 1.8 percent of GDP, higher than previously 
envisaged. The authorities' perseverance with macroeconomic stability and the relatively low public debt over the last years provide ample fiscal space. In any event, the increase in net domestic financing is expected to be temporary.

10. The BM will ease monetary policy modestly to accommodate the expansion in domestic financing of the budget. The 12-month inflation at the end of 2009 is expected to remain broadly the same as at the end of 2008 on account of a modest increase in international fuel and food prices and because of the recent depreciation of the exchange rate. The monetary framework makes room for broad money to expand for continued financial deepening, including private sector credit growth. The weak external environment will have a large adverse impact on Mozambique's external balances. As a result, the BM's gross external reserves are projected to fall by more than US\$200 million during 2009, reducing the NIR from 4.4 to 3.9 months of imports cover.

11. The recent FSAP update found considerable progress in the financial sector since 2003, though much work is needed in the areas of financial deepening. The FSAP makes recommendations in the areas of access to finance, banking supervision, and liquidity management, the non-bank financial sector (pension and insurance), and the payments system. The authorities will finalize an action plan to implement these recommendations and will form a task force to monitor implementation. BM will seek TA from the World Bank and the IMF to strengthen crisis preparedness and contingency planning, as well as improve its remedial action framework for banks in difficulty.

12. Mozambique has made considerable progress in several areas of its reform agenda. The authorities will now concentrate on activities related to the public sector reform program, in particular the wage policy, strengthening social security and the pension system, and the anti-corruption strategy. They also intend to intensify reforms to improve the business environment for the private sector, particularly the SMEs. A third strand relates to the decentralization process. In this area, the current challenge is the existing capacity at the districts for implementing financial decentralization. The authorities plan to expand the gains of PFM reforms so far at the central level to the district level and improve transparency in the allocation of resources.

13. At the end of April 2009, the authorities and the Program Aid Partners (PAPs) - the G-19 - completed the annual joint review of the general budget support mechanism. The joint review concluded that there was satisfactory performance in various areas, but both the authorities and the PAPs agreed on areas that require more attention and improvement. Thirty days later, the PAPs made their commitments for the next budget cycle 2010US\$472 million for general budget support. The authorities are grateful and encouraged by this support, particularly with the current financial crisis.

\section{Conclusion}

14. My Mozambican authorities remain committed to implementing prudent policies and persevering with the structural reforms. Mozambique is being affected by the weak economic 
performance in its major trading partners and by fluctuations in international commodity prices which is affecting the country's main exports. Considering their domestic capacity constraints, the authorities have responded to the crisis with a comprehensive set of policies and are ready to take more measures, if necessary, to sustain growth and macroeconomic stability. In this context, the authorities are mindful of both the short- and medium-term horizons when tackling the challenges arising from the current global crisis. Facing these challenges will require additional capacity. In this regard, the authorities would like to count on the support of both the Fund and other development partners to provide TA and enhanced policy discussions in the implementation of the PARPA, which would transform the economy and improve the well-being of the population. 